First published on October 06, 2011: e0144. doi: 10.1199/tab.0144

This chapter is an updated version of a chapter originally published on April 4, 2002, e0028. doi: 10.1199/tab.0028

\title{
Cytochromes P450
}

\author{
Søren Bak ${ }^{b}$, Fred Beisson ${ }^{c}$, Gerard Bishop ${ }^{d}$, Björn Hamberger ${ }^{b}$, René Höfera , Suzanne Paquette ${ }^{b, e}$, and Danièle \\ Werck-Reichhart ${ }^{\mathrm{a}, 1}$ \\ alnstitute of Plant Molecular Biology, CNRS UPR 2357, University of Strasbourg, 28 rue Goethe, F-67083 Strasbourg Cedex, France \\ ${ }^{b}$ Plant Biochemistry Laboratory, Department of Plant Biology and Biotechnology, Faculty of Life Sciences, University of Copenhagen, \\ 40 Thorvaldsensvej, DK-1871 Frederiksberg C, Copenhagen, Denmark \\ 'Department of Plant Biology and Environmental Microbiology, CEA/CNRS/Aix-Marseille Université, UMR 6191 Cadarache, F-13108 \\ Saint-Paul-lez-Durance, France \\ dDivision of Biology, Faculty of Natural Sciences, Imperial College London, SW7 2AZ \\ eDepartment of Biological Structure, HSB G-514, Box 357420, University of Washington, Seattle, WA, 98195-9420. \\ ${ }^{1}$ Address correspondence to daniele.werck@ibmp-cnrs.unistra.fr
}

\begin{abstract}
There are 244 cytochrome P450 genes (and 28 pseudogenes) in the Arabidopsis genome. P450s thus form one of the largest gene families in plants. Contrary to what was initially thought, this family diversification results in very limited functional redundancy and seems to mirror the complexity of plant metabolism. P450s sometimes share less than $20 \%$ identity and catalyze extremely diverse reactions leading to the precursors of structural macromolecules such as lignin, cutin, suberin and sporopollenin, or are involved in biosynthesis or catabolism of all hormone and signaling molecules, of pigments, odorants, flavors, antioxidants, allelochemicals and defense compounds, and in the metabolism of xenobiotics. The mechanisms of gene duplication and diversification are getting better understood and together with co-expression data provide leads to functional characterization.
\end{abstract}

\section{INTRODUCTION}

One of the surprising findings when the Arabidopsis genome sequence was completed was the extraordinary diversity of the superfamily of cytochrome P450 proteins. The final P450 count in the complete genome is 244 genes and 28 pseudogenes, which makes P450s one of the largest families of enzyme proteins in plants. Lists of $A$. thaliana $\mathrm{P} 450$ genes and accession numbers are available on several websites. Each of them provides different information:

- http://drnelson.uthsc.edu/CytochromeP450.html: provides general annotation of P450 genes in all organisms, alignments of subsets of P450 genes, and general information useful to the community of cytochrome P450 researchers (Nelson, 2009).

- http://members.shaw.ca/P450sinPlants/: provides a list of A. thaliana P450 genes with links to TAIR.

- http://arabidopsis-p450.biotec.uiuc.edu/: provides general information on plant P450s, genetic maps and mutant lists, and dedicated microarray data. A search function is incorporated in the site.

- http://www.biobase.dk/P450: is a web-based resource for Arabidopsis P450s, and family 1 glycosyltransferases (http:// www.P450.kvl.dk). It is a sequence repository of manually curated sequences, multiple sequence alignments, phylogenetic trees, sequence motif logos, 3D structures, intron-exon maps, and customized BLAST datasets (Paquette et al., 2009). This site also offers information on the P450 electron donors P450 reductase and cytochrome $b_{5}$.

- http://www-ibmp.u-strasbg.fr/ CYPedia/: provides a global heatmap of Arabidopsis P450s expression based on the Affymetrix ATH1 array analyses that can be downloaded as a poster or excel file, and a gene list with associated information on protein function, including functions already described and those predicted based on gene co-expression analysis. It also provides a list of the genes in plant metabolism co-expressed with each P450 and direct links to PLaCe / MIPS / Tair / Tigr / SIGnAL / eFP browser / ResponseViewer for each of them (Ehlting et al., 2008).

\section{NOMENCLATURE}

A common system for the nomenclature of P450 genes from all organisms has been set up on the basis of protein sequence identity and phylogeny (Figure 1; (Nelson, 2006a)). Sequence identity among P450s from Arabidopsis can be less than $20 \%$. $\mathrm{P} 450$ s in the same family usually share at least $40 \%$ identity, 


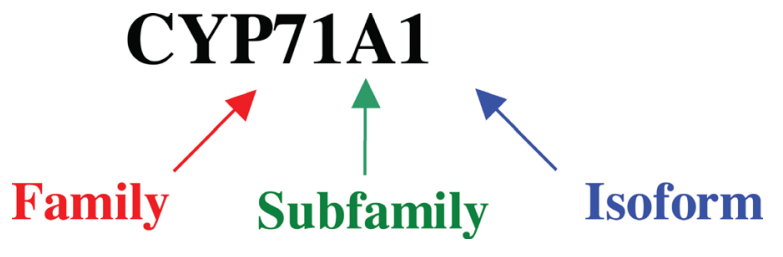

Figure 1. Naming a P450 protein.

and at least $55 \%$ identity within a subfamily. This identity rule has some exceptions, especially in plants, where gene duplication and shuffling sometimes makes a straightforward nomenclature difficult. In this case, family assignment is based on phylogeny and gene organization (for more see Nelson and Werck-Reichhart, 2011). Annotation of new genes in novel sequenced genomes can occasionally lead to the modification of a previously assigned gene name.

P450s are named in chronological order of submission to a nomenclature committee (David Nelson: dnelson@uthsc.edu). Selfassigned names are not allowed to avoid confusion and double naming. Plant P450s are being assigned names from CYP71A1 to CYP99XY, then from CYP701A1 and above.

\section{WHAT IS A P450? WHAT IS IT DOING?}

P450s are hemethiolate enzymes involved in numerous biosynthetic and xenobiotic pathways found in all organisms from bacteria to humans (Nelson, 2009). The reactions they catalyze are extremely diverse, but usually based on activation and heterolytic cleavage of molecular oxygen with insertion of one of its atoms into the substrate and reduction of the other to form water (Mansuy, 1998; Werck-Reichhart and Feyereisen, 2000; Bernhardt, 2006). They are classified as monooxygenases.

\section{$\mathrm{RH}+\mathrm{O}_{2}+\mathrm{NADPH}+\mathrm{H}^{+}$}

$\mathrm{ROH}+\mathrm{H}_{2} \mathrm{O}+\mathrm{NADP}^{+}$

Reduced P450 can bind carbon monoxide instead of oxygen. CO coordination results in a shift of the maximum of absorbance of the heme to $450 \mathrm{~nm}$ (Omura and Sato, 1964) and thus leads to the name Pigment absorbing at $\mathbf{4 5 0} \mathrm{nm}$ (P450). This property is characteristic of P450 enzymes and when $\mathrm{CO}$ is bound with high affinity it prevents binding and activation of $\mathrm{O}_{2}$. The result is an inhibition of P450 activity that can, however, be reversed by light with maximal efficiency at $450 \mathrm{~nm}$.

P450s all share a common catalytic center, heme with iron coordinated to the thiolate of a conserved cysteine (Figure 2A and B). Despite a low sequence identity at the amino acid level, P450s display a common overall topology and tridimensional fold (Figure 3) (Graham and Peterson, 1999; Werck-Reichhart and Feyereisen, 2000).

Bacterial P450s are soluble proteins, but all plant P450s described so far are bound to membranes, usually anchored on the cytoplasmic surface of the endoplasmic reticulum through
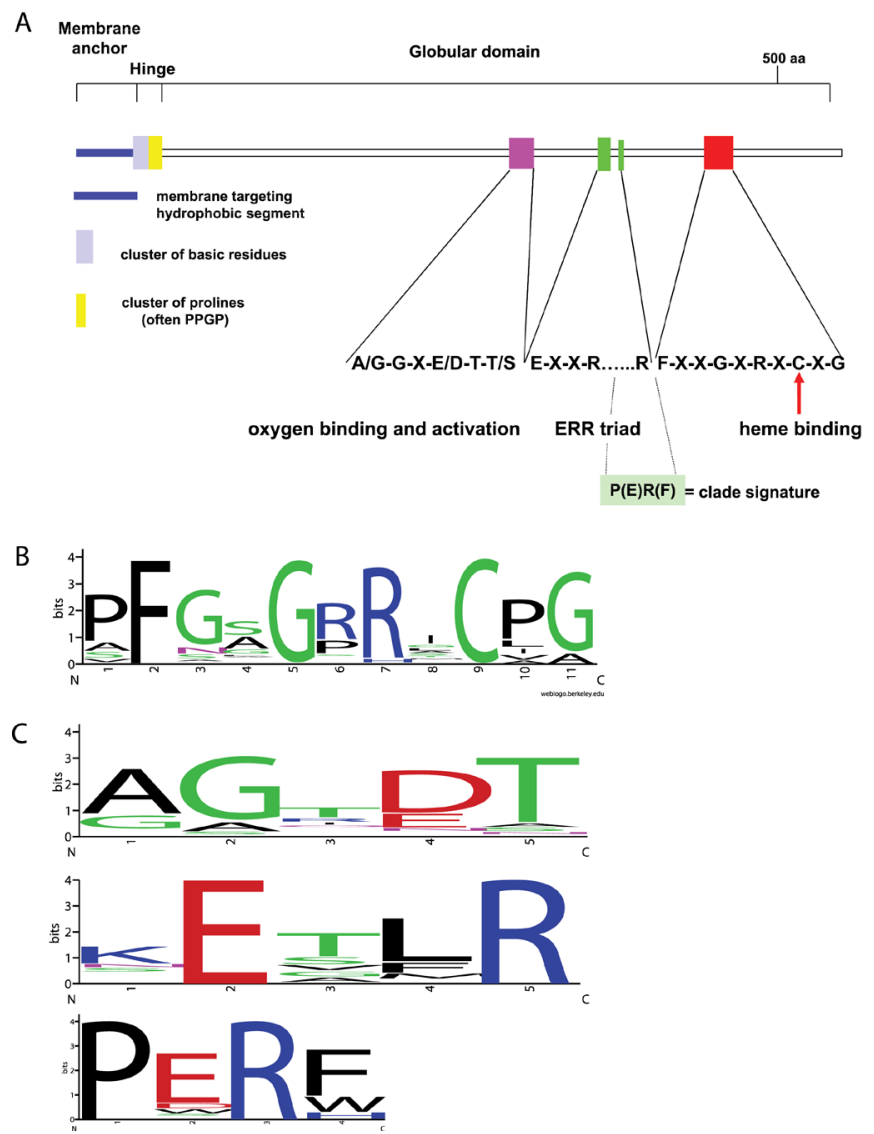

Figure 2. Conserved structures and sequences in $\mathrm{P} 450$ proteins.

(A) Map of the signature motifs in the P450 proteins. The Glu and Arg of the K-helix consensus sequence (KETLR) and the Arg in the "PERF" consensus sequence form the E-R-R triad.

(B) WebLogo of the P450 heme binding motif constructed from $A$. thaliana P450s. The letter size is proportional to the degree of amino acid conservation. The $\mathrm{F}, \mathrm{G}$ and $\mathrm{C}$ residues are conserved in all plant P450s. The $C$ residue is universally conserved in all P450s across kingdoms and coordinates the iron in the heme.

(C) WebLogos of the other conserved structures and sequences in P450 proteins. AGxDT (I-helix), KETRL (K-helix) and PERF/W, respectively.

a short hydrophobic segment of their N-terminus, and possibly a hydrophobic loop of the protein (Williams et al., 2000). However, analysis of a few Arabidopsis, and other plant P450 sequences, predicts potential signal peptides that should target some of them to the plastids or to the mitochondria (Schuler et al., 2006). This prediction has been confirmed in a few cases (for plastidial localization only: see below). To be active, P450s need to be coupled to electron-donating proteins, cytochrome $\mathrm{P} 450$ reductases or cytochrome $\underline{b}_{5}$, also anchored via their $\mathrm{N}$ - or C-terminus to the surface of the ER. Other electron donors operate in plastids or mitochondria. Electron donors for non-ER P450 proteins have not yet been identified in plants. For more on P450 reductases and the different electron-donating systems in plants and other organisms see Hannemann et al. (2007) and Jensen and Møller (2010). 
In canonical P450s, heme-bound $\mathrm{O}_{2}$ is activated by the successive transfer of two electrons from NADPH via the NADPHcytochrome P450 reductase (Figure 4). In some cases, the second electron can be provided more efficiently by an alternative electron transport chain involving $\mathrm{NADH}$, and $\mathrm{NADH}$ cytochrome $b_{5}$ reductase and cytochrome $b_{5}$ (De Vetten et al., 1999). More on the P450 redox cycle and mechanism can be found in WerckReichhart and Feyereisen, 2000 and Hamdane et al., 2008 and at http://www.p450.kvl.dk/p450.shtml. The result of P450 catalysis is very often a hydroxylated substrate, but the reaction can be more complex and lead to rearrangements of intermediates or final products (Mansuy, 1998; Isin and Guengerich, 2007; Rontein et al., 2008). Reactions catalyzed are very diverse and their number keeps growing every year. They incude among others, dealkylation, dehydration, desaturation, dimerization, C-C cleavage, ring extension, isomerization, decarboxylation and reduction. Examples of such complex reactions can be found in the list of the Arabidopsis P450 functions given below.

The natural substrates of plant P450 enzymes include precursors of membrane sterols and of structural polymers such as lignin, cutin, suberin and sporopollenin. P450s also contribute to the homeostasis of phytohormones and signaling molecules by controlling their biosynthesis (e.g. gibberellins, auxin, brassinosteroids, cytokinins, strigolactones, jasmonate) and catabolism (e.g. gibberellins, brassinosteroids, abscisic acid). They are involved in the

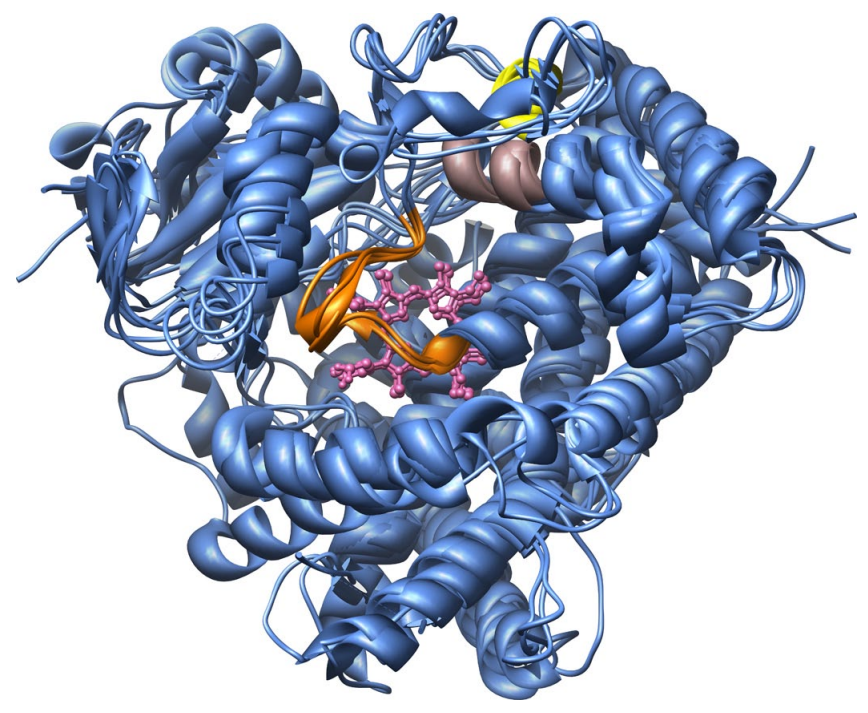

Figure 3. Consensus Matching of 4 P450s as ribbon-structures.

P450s: CYP3A4 [1TQN, Homo sapiens], CYP2B4 [1PO5, Oryctolagus cuniculus], CYP2C5 [1DT6, Oryctolagus cuniculus], and CYP2C9 [1R9O, Homo sapiens]. Color key: P450 backbone structures, blue; K-helix consensus, grey; PERF consensus, yellow; heme-binding loop, orange; heme, pink.
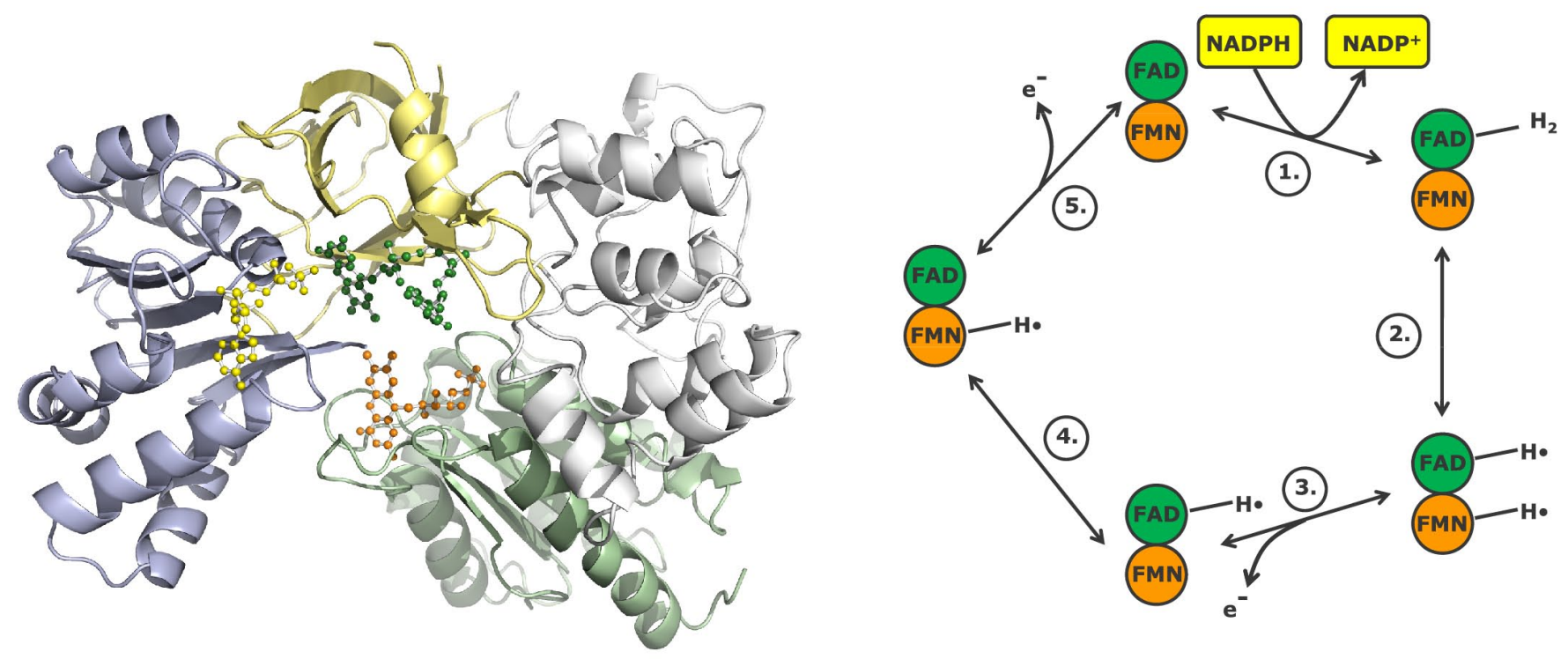

Figure 4. The NADPH-dependent cytochrome P450 reductase (CPR) is a membrane bound protein localized in the ER membrane.

CPR is a multidomain protein with three cofactor binding domains (FMN, FAD and NADPH) and a linker domain situated between the FMN and FAD/ NADPH domains. CPR donates electrons from the two-electron donor NADPH to the heme of P450 in a coupled two-step reaction as illustrated in picture on the right. NADPH binds to the NADPH-binding domain and electrons are shuttled from NADPH through FAD and FMN to the P450 heme. The CPR structure is color correlated with the reaction mechanism (http://www.p450.kvl.dk/p450rel.shtml). 
biosynthesis of pigments, volatiles, antioxidants, allelochemicals and defense compounds, including phenolics and their conjugates, flavonoids, coumarins, lignans, glucosinolates, cyanogenic glucosides, benzoxazinones, isoprenoids, alkaloids (Schuler and Werck-Reichhart, 2003; Morant et al., 2003; Mizutani and Otah, 2010). In addition to their physiological substrates, cytochromes P450 metabolize and usually detoxify xenobiotics such as pesticides and pollutants (Werck-Reichhart et al., 2000; Morant et al., 2003; Powles and Yu, 2010). More details are found below in the case of Arabidopsis and more exhaustive information on the role of P450s in different pathways can be found in the dedicated chapters of the special issue of Phytochemistry Reviews vol. 5 (2006).

\section{PHYLOGENY AND GENE ORGANIZATION}

The P450s constitute a superfamily of highly divergent sequences. To authenticate a true evolutionary relationship is not simple. Bootstrapped phylogenetic trees based on careful multiple alignments of primary amino acid sequences and characteristics such as intron position and phase, as well as a comparison of unique amino acid substitutions in highly conserved domains, can be needed for validation. Introns are lost and gained through evolution, and accordingly, intron position and phase are fossils of the evolutionary relationship that may be used as diagnostic fingerprints to validate the phylogeny predicted from the primary sequences (Long et al., 1995; Stoltzfus et al., 1997). Only a few domains are highly conserved in the primary amino acid sequence of P450s (Figure 2): the proline rich membrane hinge, the sequence surrounding the cysteine which is the axial ligand to the heme, the I-helix (AGxD/ET) involved in oxygen binding, and the E-R-R triade consisting of the Glu and Arg of the K-helix consensus sequence (KETLR) and the Arg in the "PERF" consensus sequence. The E-R-R triad is generally thought to be involved in locking the heme pockets into position and to assure stabilization of the conserved core structure (Hasemann, 1995). Of these conserved domains, only the E-R-R triad and the cysteine in the heme-binding domain are conserved in all plant $\mathrm{P} 450$ sequences.

Plant P450s were initially classified in two main clades (Figure 5): the A-type and the non-A-type (Durst and Nelson, 1995; Paquette et al., 2000). The majority of P450s involved in the biosynthesis of "secondary" metabolites seemed then to be found in the A-type group. In contrast, the non-A-type included a much more divergent group of sequences consisting of several individual clades that sometimes show more local similarity to non-plant P450s than to other plant P450s and function in lipid or hormone metabolism (Paquette et al., 2000). In all cases sequence identities of plant P450 with animal or microbial enzymes are below $30 \%$, except for the CYP51 family for which sterol demethylase function is conserved across phyla with protein sequence identity in the 30 to $40 \%$ range.

Sequencing of more plant genomes, including both more closely related and also non-vascular plant lineages, modified this initial reading of plant P450 phylogeny. It progressively appeared that the group that was initially identified as "A-type" was just the largest out of 14 P450 clans. These clans were named according to their lowest-numbered family member (Nelson et al., 2004). The initial A-type clade thus became the CYP71 clan. The ten P450 clans found in vascular plants are represented in Arabi- dopsis. Six of them contain only a single family (CYP51, CYP74, CYP97, CYP710, CYP711 and CYP727) usually required for ancestral functions such as lipid, sterol and carotenoid metabolism and signaling (Nelson and Werck-Reichhart, 2011). The four additional clans CYP71, CYP72, CYP85 and CYP86 have evolved by intensive gene duplication and diversification with a major evolutionary burst in Angiosperms. Within the latter, three families only seem specific to Brassicales (Nelson et al., 2008). Those are the CYP705 (belonging to the CYP71 clan), CYP708 and CYP702 (belonging to the CYP85 clan) families that seem to have co-evolved to form terpenoid-related pathways (Field and Osbourn, 2008).

The sequencing of the Arabidopsis genome has also revealed that $17 \%$ of the genes are organized in tandem arrays. In addition, around $60 \%$ of the genome has arisen by duplication of large chromosomal segments and ancient whole genome duplications (TAGI, 2000, Van de Peer Y. et al., 2009). Successive gene duplication events ("blooms" (Feyereisen, 2011)) in the CYP71 clan have generated expanded families during the evolutionary history. Members of the P450 (sub)families like CYP71A, CYP71B, CYP705, and CYP81 are thus organized as tandem arrays repeated on different chromosomes (Figure 6). Within the CYP71 clan, the vast majority of the genes have only a single phase 0 intron denoted as " $M$ ", signifying their close phylogenetic relationship (Figure 7). In different clans, families contain intronless genes. Those are indicitative of gene duplication mediated by reverse-transcription and retroposition. An example is the CYP94 family with a single CYP94 gene with one intron and five paralogs that are intronless (Figure 8). Another is the CYP98 family that provides the first documented case of retroposition associated to new functions in plants (Matsuno et al., 2009).

As plants are sessile organisms they have evolved to synthesize a vast number of secondary metabolites to adapt to abiotic and biotic stresses as well as to secure communication among plants. The biosynthesis of these compounds (order/genus specific pigments, odorants, flavors, antioxidants, allelochemicals and defense molecules) involves the largest and most recently diversified clans. While active gene duplication sometimes results in non-functionalization as shown by the significant number of pseudogenes, duplicates also diverge to acquire new functions (neo-functionalization). As a result, duplicated genes organized in tandem have sometimes evolved for encoding enzymes catalyzing successive steps in the same pathway. A well-documented example of sub-functionalization following gene duplication is found in the glucosinolate pathway. CYP79F1 and CYP79F2 are $88 \%$ identical at the amino acid level and positioned in tandem on chromosome 1. Both proteins are involved in biosynthesis of aliphatic glucosinolates derived from homo- to hexahomo-methionin, but with slightly different substrate preferences: CYP79F2 preferentially metabolizes long chain glucosinolates as compared to CYP79F1. As often seen for genes that have undergone neo- and sub-functionalization they are partly spatially and developmentally differentially regulated. This is also the case for CYP98A9 and CYP98A8 in tandem on chromosome 1 that catalyze two consecutive steps in the biosynthesis of phenolamides (Matsuno et al., 2009).

It is quite common that P450s belonging to single (sub)families catalyze subsequent steps in the same pathway or similar reactions in different branches of the same pathway, as illustrat- 


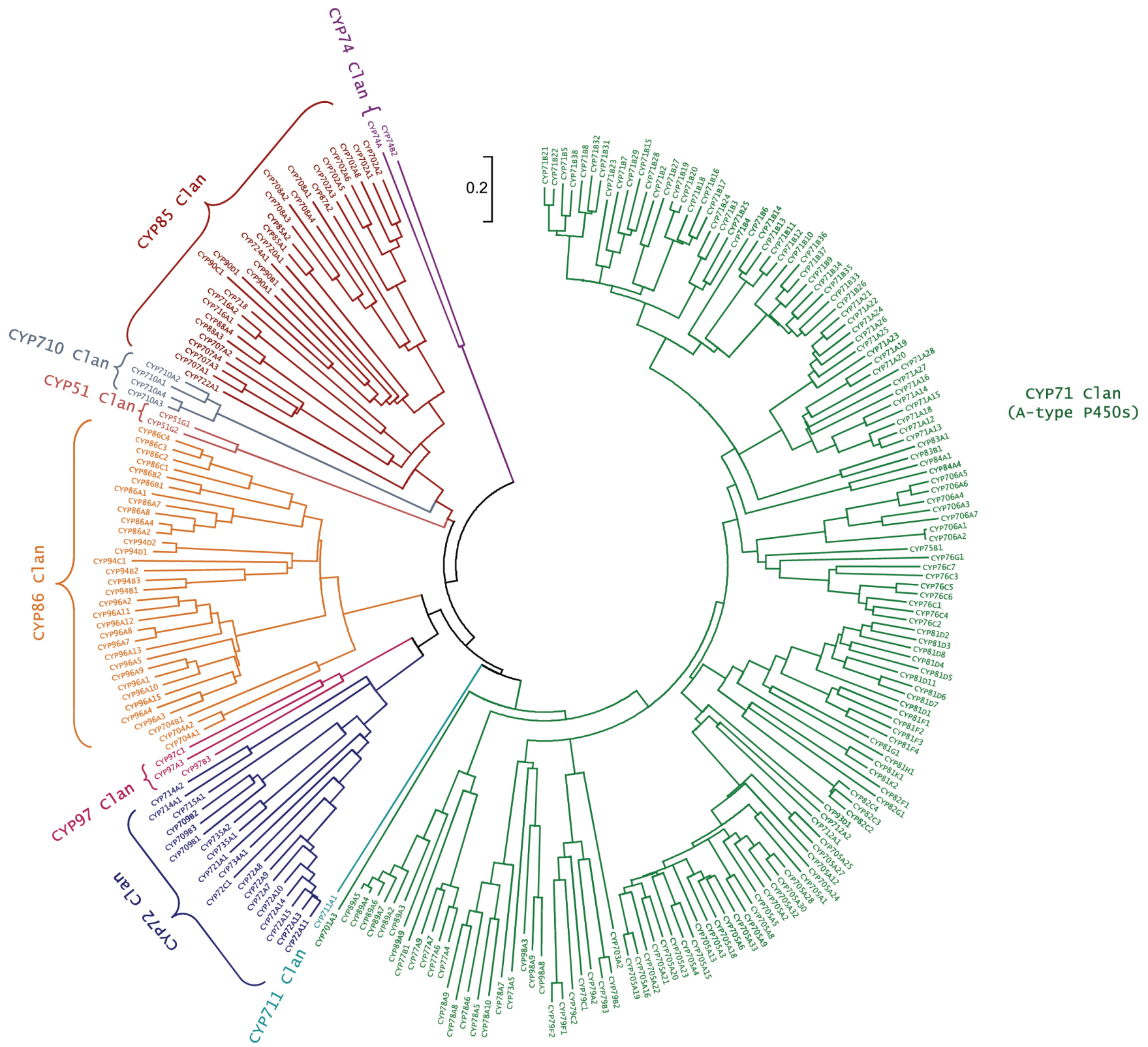

Figure 5. Circular cladogram of 246 full-length cytochrome P450s from A. thaliana.

Current software and a complete dataset have allowed resolution of the deeper branches in the non-A type P450s, and provide a clearer view of the relationships among the A type P450s (CYP71 clade). The source multiple sequence alignment was performed in MUSCLE <http://www.drive5.com/ muscle/>. Parameters were: gap opening penalty, 11; a gap extension penalty, 0.75; center, 0.0; Gonnet matrix. The alignment was refined once (identical parameters). The Neighbor-Join phylogenetic tree was created using MEGA4 <http://www.megasoftware.net/> with the Jones-Taylor-Thornton matrixbased substitution model and a 1000 bootstrap trial test (data not shown).

ed below for the CYP85 and CYP90 families in the synthesis of brassinosteroids or the CYP79 and CYP83 families in the synthesis of glucosinolates, but it is not an absolute rule. Members of the same family can be involved in different pathways. For example some members of the CYP71D subfamily have been reported to catalyze limonene hydroxylation (CYP71D13/D15 from peppermint: Haudenschild et al., 2000), and another flavone 6-hydroxyl- ation (CYP71D9 from soybean: Latunde-Dada et al., 2001). This type of functional divergence is however more unusual within the same plant species, and supports the fact that gene duplication is a driving force in speciation (Flagel and Wendel, 2009).

Accumulating knowledge of biochemical functions of individual P450s from Arabidopsis and from other plant species also challenged the original division of A- and non-A-type P450s. The 


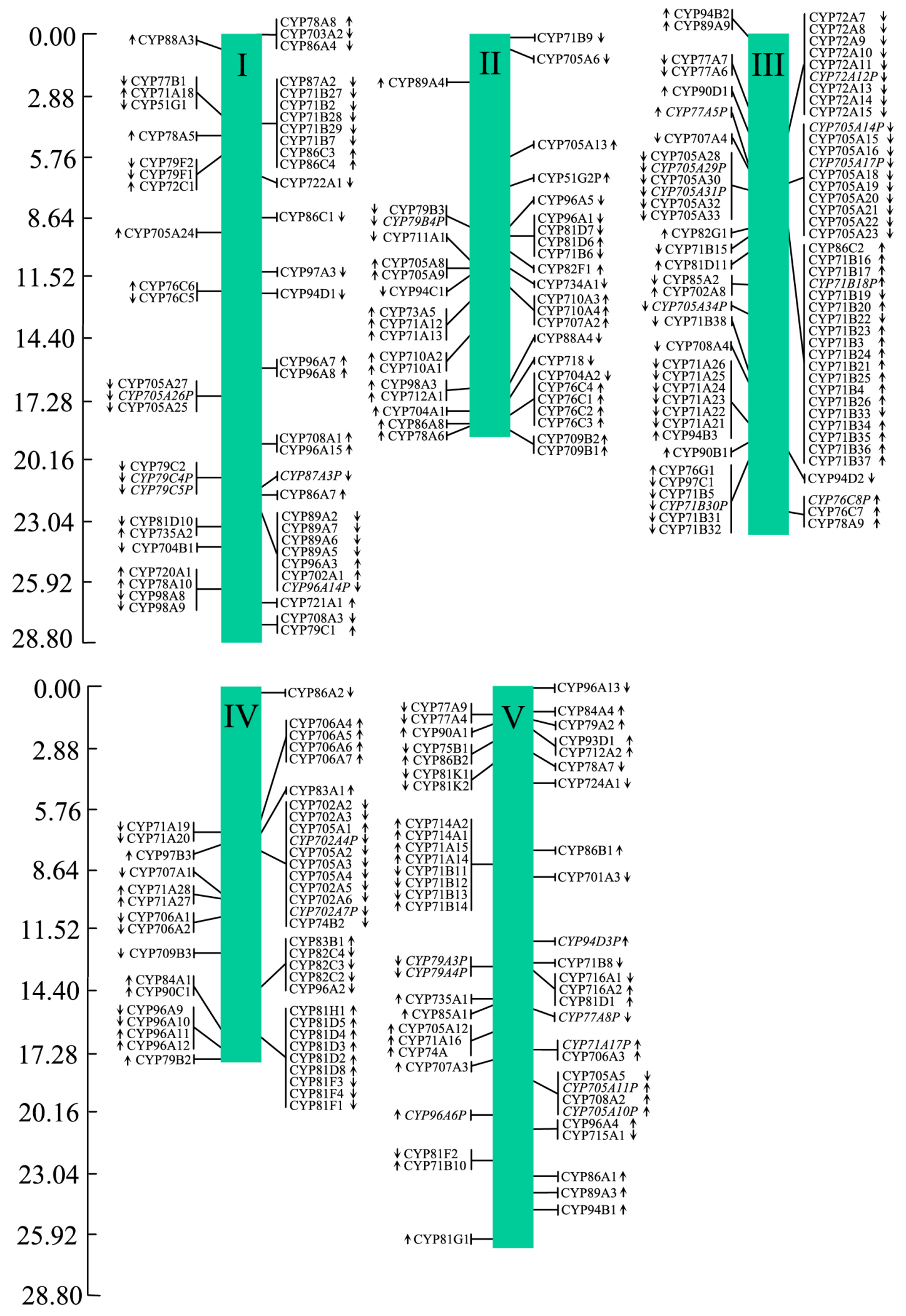

Figure 6. Genetic locations of cytochromes P450 in the Arabidopsis genome.

Chromosome Maps were made by BLAST of deduced amino acid sequence against whole chromosome sequences. Arrows indicate the relative gene orientation. Putative pseudogenes are shown in italics. 


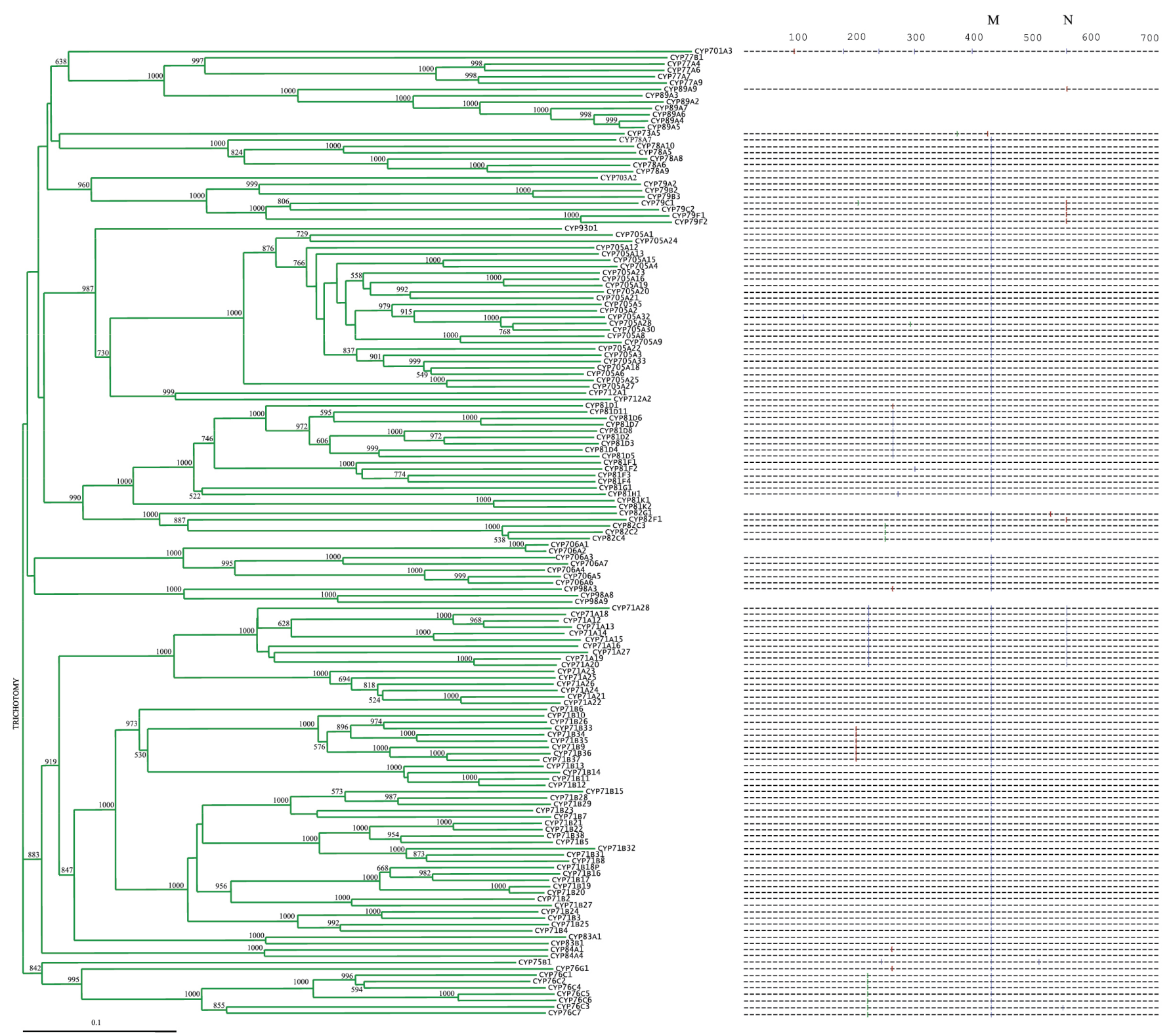

Figure 7. Neighbor-join bootstrap tree and intron map of the A-type P450s.

Bootstrap values are out of 1000 trials. Phase 0 introns: |; Phase 1 introns: [; Phase 2 introns: ]. Phase 0 introns are positioned between two codons, phase 1 introns are positioned after the first base in the condon, and phase 2 introns after the second base of the codon. P450s lacking an intronmap are intronless.

initial picture was that the CYP71 clan is primarily involved in biosynthesis of secondary metabolites and in plant specific pathways, and the non-A-type were primarily involved in metabolizing household functions, and in conserved functions essential for development and signaling. However, examples of non-A P450s involved in biosynthesis of secondary metabolites have accumulated, and conversely several enzymes belonging to the CYP71 clan have housekeeping functions, e.g. in the synthesis of plant hormones or biopolymers.
The Arabidopsis genome-sequencing project has revealed the presence of 28 P450 pseudogenes. These pseudogenes have been annotated based on the absence of a complete open reading frame due to segmented genes and to the presence of inframe stop codons or frameshifts. Microarray profiling and EST searches indicate that transcripts spanning such P450 pseudogene sequences can be found. Their significance was, however, not investigated. Moreover, alignment of P450 genes with available EST sequences revealed cDNAs extending through several 

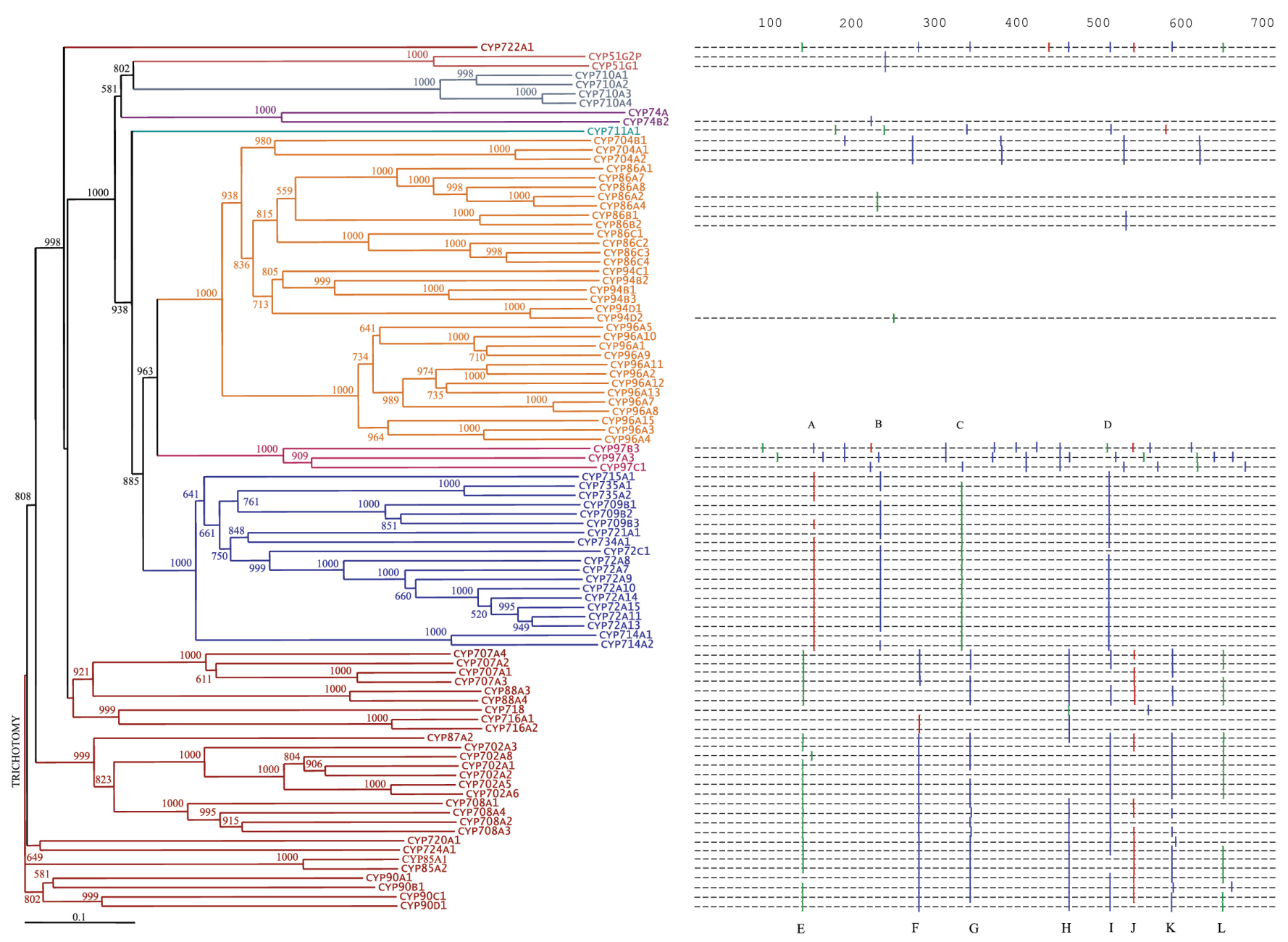

Figure 8. Neighbor-join bootstrap tree and intron map of the non-A type P450s.

P450 groups thought to comprise individual clades are colored. Bootstrap values are out of 1000 trials. Phase 0 introns: |; Phase 1 introns: [; Phase 2 introns: ]. Phase 0 introns are positioned between two codons, phase 1 introns are positioned after the first base in the condon, and phase 2 introns after the second base of the codon. P450s lacking an intronmap are intronless.

annotated transcription units (Thimmapuram et al., 2005). These include, for example, a CYP97C1/OMT bicistronic transcript encoding the two full-length proteins, or two types of differentially spliced bicistronic transcripts spanning CYP705A15 and CYP705A16, one of them encoding the two proteins deleted from a short 28 and 31 amino acid segment at their $\mathrm{N}$-terminus, respectively. Another encodes a fusion protein of CYP71B10 with eight pentatricopeptide repeat motifs. In the case of CYP96A9/CY$\mathrm{P} 96 \mathrm{~A} 10$, a transcript is found that encodes a dimeric $\mathrm{P} 450$ protein. In all these cases, the existence of the extended transcripts was confirmed by RT-PCR amplification. They were usually found in the plant in conjunction with the monocistronic transcripts.

The alignments (including marked intron positions and phase) behind the trees presented in Figures 7 and 8 , as well as unrooted trees, can be found at http://www.biobase.dk/P450:
Non-A type P450s: There are 93 non-A P450s and 7 putative non-A pseudogenes (Figure 8). In the non-A P450 tree published in 2000 by Paquette et al., four branch-points were identified, based on bootstrap values and intron conservation, but the tree could not resolve all of the deeper branches. The completion of the Arabidopsis genome has provided 32 additional non-A sequences, and now enables a more complete analysis of the paralogous non-A Arabidopsis P450s.

CYP97s are among the most ancient plant CYPs and are already present in Chlamydomonas (Nelson and Werck-Reichhart, 2011). They form a single-family clan. All three subfamilies in CYP97 are represented in all plants, usually in single copy, which indicates critical functions. This is not surprising, considering that this function is related to synthesis of carotenoids (see CYP97 family below). 
The CYP74 family also constitutes its own clade. It is more ancient than plants and is detected in Rhizobacteria, Cnidaria and Chordata (in amphioxus) (Lee et al., 2008). Its evolution in plants is still a matter of debate (Nelson and Werck-Reichhart, 2011). The primary sequence of CYP74 differs significantly from other plant P450s and hence the position of CYP74 in the tree is not strongly fixed. CYP74 members do not utilize molecular oxygen or a redox partner. Oxygen, reducing equivalents and protons needed for the reaction are all brought by the fatty acid hydroperoxide that is used as a substrate. Accordingly, it is not surprising that the otherwise conserved residues in the oxygenbinding domain are absent and only the cysteine residue in the heme-binding domain is present. The E-R-R triad is conserved in the CYP74 family.

CYP711 is one more single-family clade that does not group strongly with any other in Arabidopsis. The CYP711 clan appears early in plant evolution and is represented by two divergent most likely ancestral families in green algae (Nelson and Werck-Reichhart, 2011). CYP711 family appears in Lycopods and is kept as a single copy gene in most dicots but not in monocots. It does not share intron conservation with other P450s from Arabidopsis. As explained below, its function seems related to the synthesis of the carotenoid-derived hormones strigolactones (Gomez-Roldan et al., 2008; for more see CYP711 section).

CYP51 and CYP710 form individual clades. Both CYP51 and CYP710 clans are very ancient and are already present in Chlamydomonas, but are also in brown algae and fungi (CYP710 ortholog is CYP61 in fungi) (Nelson and Werck-Reichhart, 2011). CYP51 is also present in animals (except for in Arthropods that rely on their diet for sterol supply). In all organisms, they are required for the synthesis of membrane sterols. They seem to be at the origin of the CYP85 clan. This is supported by clustering (Figure 5), the order of appearance of the genes in evolution and their related enzyme activities on sterols, steroids or triterpenes, but is not supported by intron position. The CYP710s lack introns, while the CYP51s have a single intron and the CYP85 clan members have several highly conserved introns, which is different from CYP51.

The CYP85, CYP87, CYP88, CYP90, CYP702, CYP707, CYP708, CYP716, CYP718, CYP720, CYP722, CYP724 and CYP728 families form the CYP85 clan. Ancestral members of this clan appear early in plant evolution (green algae), but the families still found in Angiosperms emerge in the liverworts or bryophytes for the first time (CYP88, CYP716; Nelson and Werck-Reichhart, 2011). Bootstrap values, conservation of intron positions, and the change of the PERF consensus into $P(W / S) R W$ validates this clan. As detailed below, members of this clan are involved in the metabolism of both general (i.e. primary) as well as specialized (i.e. secondary) terpenoids.

The CYP86 clan, formed of the CYP86, CYP94, CYP96 and CYP704 families, appears later in plant evolution, but CYP86, CYP94 and CYP704 families are present in moss (Nelson and Werck-Reichhart, 2011). CYP96 and CYP94 family members are generally intronless, whereas some of the CYP86 genes contain unique introns. CYP704s have up to four or five introns. The generally highly conserved PERF motif has a Phe to Trp substitution, so that members of the CYP86, CYP94, CYP96, and CYP704 families have the consensus sequence PERW. As detailed below, the function of these members of the CYP86 clan is related to fatty acid and alkane metabolism.
CYP72, CYP734, CYP714, CYP721, CYP709, CYP735, and CYP715 form the CYP72 clan. The first members of this clan are present in liverworts, but families present in Angiosperms appear only in ferns (CYP72, CYP709 and CYP735; Nelson and WerckReichhart, 2011). This clade is characterized by four highly conserved introns. Intron position and phase are highly conserved in this clade and bootstrap values are generally high. As detailed below, the members of this clade have functions reported in fatty acid metabolism, cytokinin biosynthesis and catabolism of hormones (gibberellins and brassinosteroids).

A-type P450s: There are 152 A-type P450s, and 20 putative A-type pseudogenes in Arabidopsis (Figure 7). They all belong to a single monophyletic clan CYP71. The families of this clan, CYP73, CYP98 and CYP736, are first found in Marchantia (Nelson and Werck-Reichhart, 2011) and more maybe found when Marchantia's full genome sequence is available. This huge clade results from successive gene duplications and family blooms. The CYP71 family is the best illustration of this duplication and bloom process with 52 members in the Arabidopsis genome. The monophyletic origin of the CYP71 clan is attested by a single highly conserved intron, $M$, which is not found in any of the nonA-type P450s. Within the different CYP71 clan families and subfamilies, some additional conserved introns are seen. However, the number of introns tends to be than those seen in the nonA P450s. Due to nomenclature rules, the names of the individual families usually follow the phylogenetic grouping. An exception is the CYP83 family that should be regarded as belonging to the larger CYP71 clade. It was decided not to rename CYP83A1 and CYP83B1 to avoid nomenclature ambiguities with regard to published data <http://drnelson.uthsc.edu/CytochromeP450.html>.

Members of the CYP79, CYP703 and CYP71A families have a Phe to His substitution in the PERF domain. CYP703 clusters with a bootstrap value of 960/1000 to the CYP79s whereas the CYP71As and CYP79s are clearly not closely related. The branch is deep indicating that CYP79s and CYP703s may be distantly related phylogenetically.

Four families, CYP73, CYP77, CYP89 and CYP701, are difficult to place in the CYP71 clan phylogenetic trees. Their intron-exon boundaries are also different from other genes in the CYP71 clan. These families contain PERF and heme sequences that more resemble the consensus of core A-type P450 members such as the CYP705 and CYP71 families, rather than the consensus found in non-A-type clades. CYP73 lacks the conserved M intron and does not share its two introns with any other CYP71 clan gene. Analyses with both ClustalX and MEGA place CYP73 more or less randomly in the A-type clade. The members of families CYP77 and CYP89 form a strong cluster with a bootstrap confidence of $990 / 1000$ and they are generally characterized by being intronless, thus lacking the conserved $M$ intron. CYP701A3 is an unusual A-type P450 as it contains 6 unique introns. Despite their atypic intron organization, the four families clearly cluster within the CYP71 clan (Figure 5; Nelson and Werck-Reichhart, 2011).

\section{GLOBAL APPROACHES TO ARABIDOPSIS P450 FUNCTIONS}

The large number of P450 genes revealed by the sequencing of the Arabidopsis genome highlighted that we knew very little of the metabolism occuring in this plant. It also suggested that focused 
large-scale approaches might help to speed-up the determination of the function of the more than $80 \%$ of orphan $\mathrm{P} 450$ genes (i.e. P450s without known function). Several strategies have thus been proposed to screen for or predict P450 functions.

Dedicated oligonucleotide DNA microarray analyses were initially undertaken that provided preliminary information on their expression profiles and revealed some organ-specific expression and differential stress responses, thereby confirming that gene duplication led to functional diversification (Xu et al., 2001; Schuler et al., 2006: http://arabidopsis-p450.biotec.uiuc.edu/microarray.shtml). In a genome-wide co-expression study by mining broader publicly available microarray expression data, Ehlting and co-workers (2008) have correlated P450 expression with the expression of genes predicted to be involved in the different branches of plant metabolism. This analysis is available at http:// www-ibmp.u-strasbg.fr/ CYPedia/. The website provides extensive information on P450 gene expression in different organs and tissues, upon pathogen, stress and hormone response, and in mutants. It also scores co-expressed genes in plant metabolism and proposes pathway predictions. Several of these predictions have already been validated, in terpenoid, lipid and phenolic metabolism (Field and Osbourn, 2008; Sauveplane et al., 2009; Matsuno et al., 2009). One of the most striking findings emerging from this study is that an unexpectedly large group of 79 orphan P450 genes with unknown function was mapped to pathways identified as "plastidial isoprenoids", together with two of the three P450s of the CYP97 family reported to hydroxylate the $\beta$ - and $\varepsilon$-rings of carotenoids (see below). Consistently, manual curation of in silico predicted subcellular localisation allowed Schuler and coworkers (2006) to identify eleven chloroplast P450s, including the CYP97 family, which may indicate a potential function in the metabolism of plastid-derived compounds. Up to 98 P450 loci showed circadian regulation for at least one photoperiod condition (Pan et al., 2009), which may be partially related to plastidial localization or just due to regulation correlated with plastidial functions.

The second global approach to gene function that was proposed was a screening for enzyme activity. Functional screening of P450 enzymes is complicated by their dependence on an NADPH-cytochrome reductase to provide electrons required for oxygen activation and catalytic activity. In addition, both the P450 and reductase are membrane-anchored proteins. Activity screening thus requires a natural or artificial membrane system encompassing both the P450 and the reductase. This can be most easily obtained in a recombinant plant or microorganism. One of the strategies that has been validated used microsomal membranes prepared from a collection of yeast-expressed enzymes (recombinant yeasts co-expressing P450s and P450 reductase). Detection of P450 activity was based on microplate fluorescencemonitoring of oxygen consumed by the reaction (Olry et al., 2007). A small-scale test-screening carried out using this method with six yeast-expressed enzymes and 80 candidate substrates led to the demonstration that an orphan Arabidopsis P450, CYP72A8, catalyzes the hydroxylation of quinidine, a major component of the most common antimalarial drugs. This approach can however be hampered by uncoupling or by the presence of natural substrates (e.g. lipids) in the recombinant cell membranes. This screening system was very effective for the identification of new P450 inhibitors. Kruse and coworkers (2008) tested another strategy using Arabidopsis over-expression lines. Seedlings from a subset of 91 Arabidopsis WS-2 lines stably transformed with 35S:P450 constructs were grown in 24-well plates with 101 candidate substrates and subsequently extracted and analyzed via LC/MC. This led to the demonstration that 8-methoxypsoralen was a substrate for members of the CYP82C subfamily and that the 5-hydroxylated product was further glycosylated by the plant. This approach is however limited by substrate uptake and also by the extraction and analytical methods chosen for the detection of the product(s).

\section{ARABIDOPSIS P450 FAMILIES: EVOLUTION, FUNCTION AND REGULATION \\ CYP51}

Two CYP51 copies are present in Arabidopsis. CYP51 is the only P450 family conserved across evolution within fungi, animals and plants. A few bacterial genomes also encode a CYP51, but the current view is that bacterial CYP51 genes originate from horizontal transfer from eukaryotes (Rezen et al., 2004). CYP51 is considered as an essential gene in all eukaryotic organisms since it controls de novo synthesis of sterols for membranes, needed in low amounts for meiosis and cell cycle activation. CYP51s catalyze the essential $14 \alpha$-demethylation step in biosynthesis of sterols by three consecutive hydroxylations at the $\mathrm{C} 14$ position followed by elimination of formic acid. The introduction of the double bound is essential as it serves to flatten the sterol backbone stucture to enable proper insertion in membranes. CYP51 is absent in nematodes and Arthropods, as they are sterol heterotrophs and CYP51 has become superfluous and extinct over time in these organisms. Although substrates and pathway structures differ in fungi, animal and plants, the CYP51s from different phyla are considered as orthologs. The CYP51 substrate in plants is obtusifoliol (Figure 9), while in fungi and animals the substrate is lanosterol, dihydrolanosterol or eburicol. The plant CYP51 catalytic function has been validated in vitro with the recombinant enzymes of wheat and sorghum (Cabello-Hurtado et al., 1997; Bak et al., 1997).

CYP51 is duplicated in Arabidopsis. The two genes, initially named CYP51A1 and CYP51A2, encode proteins $72 \%$ identical at the amino acid level. While CYP51A1 is predominantly expressed in roots, CYP51A2 is evenly expressed in all tissues (Kim et al., 2005a), but CYP51A1 has since been identified as

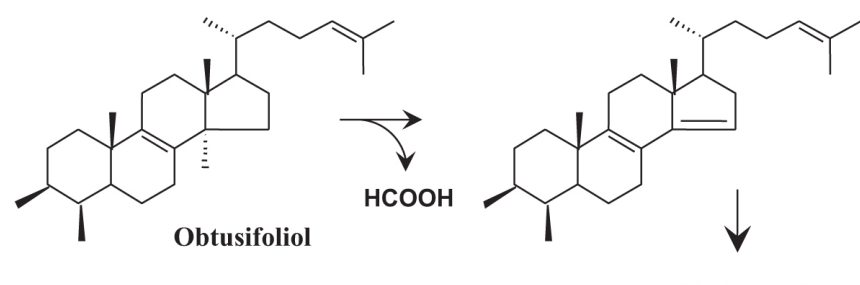

Phytosterols

Figure 9. The reaction catalyzed by CYP51 genes in higher plants.

CYP51 is an obtusifiliol $14 \alpha$-demethylase and catalyzes three successive oxidations of the carbon 32 , leading to $\mathrm{C}-\mathrm{C}$ rupture and release of the methyl in C-14 as formic acid to form $4 \alpha$ methyl-5aergosta-8,14,24(241)trien- $3 \beta$ ol. 
an expressed pseudogene. Since the original naming of the two Arabidopsis CYP51s, their subfamily has been changed to CYP51G. This new subfamily designation indicates if the CYP51 is from plants, animal, fungi, bacteria or from another phylum ( $\mathrm{Nel}-$ son et al., 2004). Accordingly, CYP51A is now reserved for sterol demethylase from animals and CYP51G for sterol demethylase from green plants and other Archaeplastida (including red algae and glaucophytes). CYP51H designates a functionally divergent subfamily present only in monocots. Thus Arabidopsis CYP51A1 is now called CYP51G2P, and CYP51A2 is CYP51G1.

Loss-of-function mutants for CYP51G1 shows multiple defects, such as stunted hypocotyls, short roots, reduced cell elongation, seedling lethality and accumulation of obtusifoliol. The lack of proper sterol composition in the mutant leads to defects in membrane integrity and hypocotyl elongation underpinning the essential role of CYP51G1 in controlling plant growth and development by a sterol-specific pathway (Kim et al., 2005a).

\section{CYP71}

CYP71 emerges as the largest P450 family in all plant species, showing large clusters of duplicated genes and taxa-specific subfamily blooms (http://drnelson.uthsc.edu/P450dbplant.html; see also Nelson and Werck-Reichhart, 2011). The CYP71A and CYP71B subfamilies have bloomed in the Arabidopsis genome, in addition to CYP83s, with 17 CYP71As and 37 CYP71Bs. CYP71Bs form the largest P450 cluster with 13 of them found in tandem on chromosome III.

The first plant $\mathrm{P} 450$ gene isolated was from ripening avocado and belongs to the CYP71A subfamily (Bozak et al., 1990). The recombinant enzyme was shown to have monoterpene hydroxylase activity (Hallahan et al., 1992), but its function needs to be confirmed in planta. Of the 54 CYP71s in Arabidopsis only the functions of CYP71A12, CYP71A13 and CYP71B15 have been investigated. CYP71A13 has been shown to catalyze the conversion of indole-3-acetaldoxime to indole-3-acetonitrile (IAN) in the synthesis of the brassicaceae-specific phytoalexin camalexin (Nafisi et al., 2007). Camalexin is an indole alkaloid phytoalexin produced by $A$. thaliana and is important for resistance to necrotrophic fungal pathogens, such as Alternaria brassicicola and Botrytis cinerea. Plants carrying cyp71a13 mutations produce greatly reduced amounts of camalexin after infection by Pseudomonas syringae or A. brassicicola and are susceptible to A. brassicicola. In the same line, it was recently shown that CYP71A12, the tandem duplicate of $C Y P 71 A 13$, is essential for camalexin formation in roots (Millet et al., 2010). CYP71A12 expression is highly induced by the Flg22 elicitor in Arabidopsis roots.

The pad3 (phytoalexin deficient3) mutation in Arabidopsis also causes a defect in the biosynthesis of camalexin and was, as early as 1999, associated with the CYP71B15 gene (Zhou et al., 1999). In vitro analysis of heterologously expressed CYP71B15 showed that it is a multifunctional P450 that catalyses the conversion of cysteine-indole-3-acetonitrile to camalexin, and thus succeeds CYP71A13 in the camalexin pathway (Böttcher et al., 2009). CYP71A13 and CYP71B15 expression is co-regulated, and both transcripts accumulate in infected or salicylic acidtreated plants, but not in mutants affected in regulation of camalexin biosynthesis. The functions of CYP71A12, CYP71A13 and
CYP71B15 add to the complexity in tryptophan-derived metabolism in Arabidopsis, which is still not fully understood. CYP79B1 and CYP79B2 catalyze conversion of tryptophan to indole-3-acetaldoxime, which is a branchpoint in biosynthesis of the phytohormone indole-3-acetic acid (IAA) and the two major defense compounds in Arabidopsis: indole glucosinolates and camalexin. Furthermore IAN, which can be produced by CYP71A13, probably by CYP71A12, and/or by degradation of indole glucosinolates, is an intermediate in camalexin biosynthesis and may also be a substrate for IAA biosynthesis.

Other CYP71 subfamilies are involved in the biosynthesis of indole phytoalexins (CYP71C), terpenoids and flavonoids (CYP71D), cyanogenic glucosides (CYP71E), mono- and sesquiterpenoids (CYP71AR, CYP71AV, CYP71BA and CYP71BL), furanocoumarins (CYP71AJ) and alkaloids (CYP71BJ). As these are absent in the Arabidopsis genome and also quite distantly related to CYP71As and CYP71B subfamilies they are not reviewed.

\section{CYP72}

The CYP72 family was originally classified as having three subfamilies in Arabidopsis with nine CYP72A genes, one CYP72B and one CYP72C. The CYP72As are present as a large cluster of 8 genes and one pseudogene on chromosome III suggesting recent gene duplication events. CYP72B1 has been renamed as CYP734A1 and is a brassinolide 26-hydroxylase, involved in the catabolism of brassinosteroid hormones (see CYP734A section). The function of CYP72As in Arabidopsis is unknown. CYP72A13 is upregulated upon application of cis-jasmone and is correlated with increased levels of 4,8,12-trimethyl-(EE)-trideca-1,3,7,11tetraene (TMTT), although analysis of lines over-expressing CYP72A13 could not confirm increased TMTT production (Bruce et al., 2008). CYP72A1 from Catharanthus roseus is a secologanin synthase, the ring-opening enzyme in the biosynthesis of the seco-iridoid unit of terpene indole alkaloids (Irmler et al., 2000). A medium-throughput functional screening for Arabidopsis P450 catalytic activity recently revealed significant quinidine hydroxylase activity of CYP72A8 (Olry et al., 2007). This is unlikely to be the function of the enzyme in the plant. It may however provide a lead to the correct substrate and a tool for further drug functionalization.

CYP72C1 seems to be restricted to Brassicaceae, as no obvious orthologs have been detected in other plant species, except for papaya. CYP72C1 is involved in plant steroid hormone (brassinosteroid, BR) inactivation, although its exact role in BR metabolism has yet to be defined. Spectral analysis of recombinant CYP72C1 indicates that it binds brassinolide precursors and thus acts upstream of CYP734A1 (Thornton et al., 2010). Activation tagged mutants of this P450 in Arabidopsis include chibi2, sob7 and shk1 (Nakamura et al., 2005; Takahashi et al., 2005; Turk et al., 2005). These mutants have over-expression of CYP72C1 resulting in a dwarf phenotype similar to BR-deficient mutants (see Figure 10). The chibi2 dwarf mutants have reduced levels of both 6-deoxocastasterone and castasterone (Takahashi et al., 2005). CYP72C1, unlike CYP734A1, is not transcriptionally regulated by BRs (Takahashi et al., 2005). The highest level of expression of CYP72C1 is associated with siliques (Takahashi et al., 2005; Turk et al., 2005). 


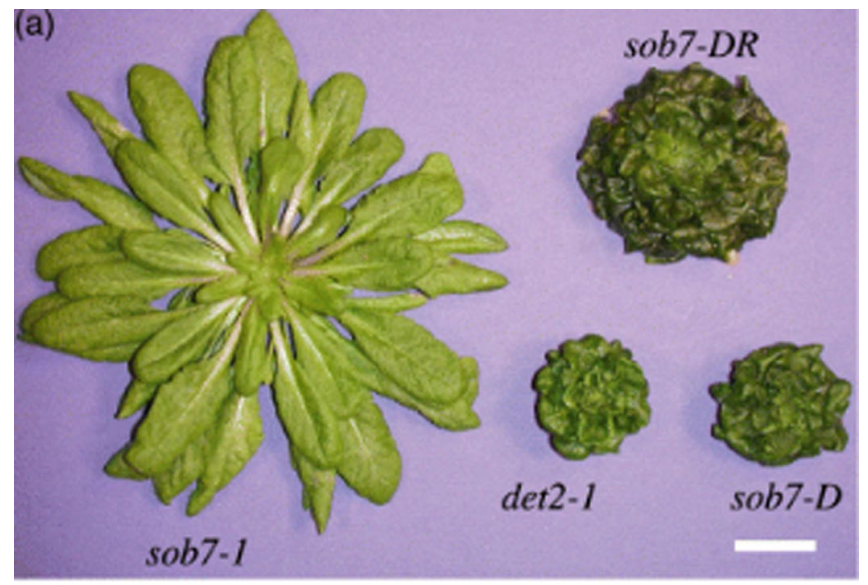

Figure 10. Activation-tagging identifies an Arabidopsis BR-deficient mutant.

The activation-tagged mutant sob7-D is similar to a BR-deficient mutant (det2-1) and the sob7-DR recapitulation line. SOB7 loss-of-function line (sob7-1) is similar to the Col-0 wild type (data not shown). Plants were grown for 9 weeks in short days. Image reprinted from Turk et al. (2005) with permission from Wiley-Blackwell Publishers.

\section{CYP73}

CYP73A5 is the cinnamic acid 4-hydroxylase $(\mathrm{C} 4 \mathrm{H})$, and the only CYP73 family member in Arabidopsis. The enzyme catalyzes the second step of the phenylpropanoid pathway (Figure 11). CYP73s were the first plant P450 genes to be associated with a specific biochemical and physiological function. This pioneering work was not carried out in Arabidopsis (for review see Ehlting at al., 2006).

The catalytic function of CYP73A5 from Arabidopsis was confirmed by expression of the cDNA in insect cells (Mizutani et al., 1997) and successfully assembled as a functional catalytic unit with a housefly P450 reductase in lipid nanodiscs (Duan et al., 2004). Substrate specificity was further explored after expression in yeast (Chen et al., 2007) to show that 3-coumaric acid and various orthosubstituted cinnamate analogs were metabolized by the enzyme. In addition a tentative model of the three-dimensional structure of the CYP73A5 active site has been proposed by Rupasinghe et al. (2003) based on homology modeling to the crystal structures of four soluble bacterial P450s and one mammalian P450.

The CYP73A5 gene and its promoter have been isolated by two groups (Bell-Lelong et al., 1997; Mizutani et al., 1997). The CYP73A3 promoter has repeatedly been found much more efficient than the CaMV $35 S$ promoter for driving the expression of genes, such as coniferaldehyde 5-hydroxylase, in lignin biosynthesis in different plant species (Meyer et al., 1998; Franke et al., 2000) which identified it as a useful tool for lignin biotechnology. The coding sequence contains two introns conserved in most CYP73 sequences reported so far. They do not align with the highly conserved " $M$ " intron in the other A-type P450 genes. The Arabidopsis ecotype Colombia and Lansdberg erecta alleles of CYP73 differ in 13 positions of the coding sequences, 12 of which are silent and one results in a $\mathrm{H} 92$ to $\mathrm{L}$ substitution in the Columbia ecotype (Bell-Lelong et al., 1997).<smiles>O=C(O)/C=C/c1ccccc1</smiles><smiles>CCCCCCCCCCCCCCCC(=O)O</smiles>

trans-Cinnamate

p-Coumarate

Figure 11. The reaction catalyzed by CYP73A5.

Both the above mentioned groups investigated CYP73A5 regulation and showed that its expression pattern was essentially the same as that of other genes in the core phenylpropanoid pathway, such as those coding for phenylalanine ammonia-lyase and 4-coumarate CoA ligase (Bell-Lelong et al., 1997; Mizutani et al., 1997). Transcripts were detected in all plant tissues, but were highest in stems, roots and siliques. Transcription increased within 1 hour of light exposure or wounding. In agreement with these data, various enhancer sequence boxes (P, A, G, H, L, AC-I, ACIII, 1, 2 and 3) previously identified in other genes of the phenylpropanoid pathway were also found in the promoter of CYP73A5. Tissue-specific expression, analyzed with GUS translational fusions, was detected strongly in all vascular tissues from stems, roots and leaves, in young shoot meristems and in reproductive tissues (Bell-Lelong et al., 1997). Expression was induced by wounding of mesophyll and parenchyma cells from mature leaves (Figure 12). Expression of CYP73A5 also appeared to increase in etiolated roots upon exposure to light (Hemm et al., 2004) for the production of coniferyl and sinapyl glucose conjugates. Overall CYP73A5 expression was not significantly altered in $t t$, fah1-2 or sng1-1 mutant backgrounds from 8-day-old plants, except in the case of the $t t 8$ mutant (Bell-Lelong et al., 1997). Thus strong perturbations in phenypropanoid metabolism do not seem to result in large changes in CYP73A5 expression. Another study provides evidence of regulation of CYP73A5 dissociated from that of other genes of the phenylpropanoid pathway in response to UV-B irradiation (Jin et al., 2000). This study demonstrates that CYP73A5 is the principal target of AtMYB4, a transcriptional repressor down-regulated in plants exposed to UV-B. Regulation of the synthesis of sinapate ester sunscreens may thus occur at the level of CYP73A5. The MYB4 protein binds its own promoter for repression of its own expression to form a feedback loop. The SAD2 nuclear importin b-like protein is required for MYB4 nuclear import and CYP73 repression (Zhao et al., 2007).

Due to the essential function of CYP73 for plant development it took a quite long time until mutants for this gene were described (Schilmiller et al., 2009). As mentioned by these authors, analysis of a $\mathrm{GABI}$ insertional line revealed that null mutants display a seedling lethal phenotype, with seedlings germinating but primary leaves failing to expand. A series of allelic EMS mis-sense and leaky mutants was therefore isolated based on reduced epidermal fluorescence (ref3 mutants: Ruegger and Chapple, 2001; Schilmiller et al., 2009). All these mutations induced single amino acid substitutions in conserved regions and substrate recognition sites (SRS) of the protein and resulted in enzyme instability or improper folding. Severity of the phenotype depended on the allele, ranging from just loss of apical dominance to severe dwarf- 


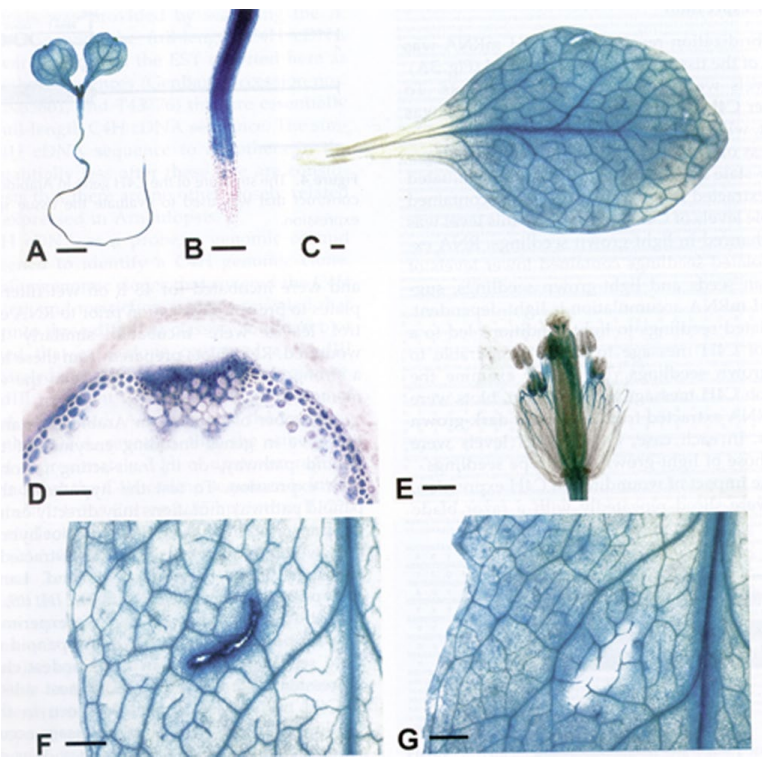

Figure 12. In vivo GUS staining in CYP73A5 promoter:GUS transformants. Image reprinted from Bell-Lelong et al. (1997).

(A) Ten-day-old seedlings; (B) 10-day-old seedling root; (C) mature leaf; (D) rachis transverse section; (E) flower; (F) mature leaf stained $48 \mathrm{~h}$ after wounding; (G) mature leaf stained immediately after wounding. A, C, E, F, $\mathrm{G}, \mathrm{Bar}=500 \mathrm{~mm} . \mathrm{B}, \mathrm{D}, \mathrm{Bar}=10 \mathrm{~mm}$.

The CYP73A5 promoter was successfully used to drive the expression of the coniferaldehyde 5-hydroxylase (CYP84A1) gene in the vascular tissues from Arabidopsis and tobacco (Franke et al., 2000; Meyer et al., 1998).

ness and complete male sterility (Figure 13). The latter resulted from failed dehiscence, but mainly from complete pollen abortion, probably for a large part due to a deficiency in sporopollenin and pollen coat deposition. Strong alleles in addition presented unusual swellings at the base of lateral branches formed of large and disordered cortical cells. At the molecular level, mutants displayed a strong decrease in lignin content with increased syringyl to guaiacyl ratio (unchanged syringyl versus guaiacyl decreased by half). Stronger alleles resulted in collapsed xylem in tracheary elements. Analysis of soluble metabolites also revealed that mutants accumulated cinnamoyl malate instead of sinapoyl malate as the main soluble phenolic. Such data are in agreement with the position of CYP73/C4H upstream in the phenylpropanoid pathway and with an essential function in the plant's ontogeny. For more on see also Fraser and Chapple (2011).

\section{CYP74}

CYP74s are the most atypical plant P450s. They act in the absence of a NADPH-dependent P450 reductase or any other electron transfer system and do not activate molecular oxygen (i.e. they do not function as monooxygenases). Instead, CYP74s catalyze the conversion of already oxygenated polyunsaturated C18 fatty acid hydroperoxides into other oxylipins. Besides plants,
CYP74s occur in bacteria and basal animals (Lee et al., 2008). They may represent an intermediate in the evolution of P450 catalysis from an $\mathrm{H}_{2} \mathrm{O}_{2}$ to an $\mathrm{O}_{2}$ oxygen donor.

As expected from their unique non-monooxygenase activity, CYP74s significantly differ from other plant P450s in their primary sequences (Paquette et al., 2000). The residues involved in oxygen binding and activation, or electron transfer, are absent from their sequences, which also have unusual N-termini with putative chloroplast transit peptides. The sequence alteration in the oxygen-binding region results in a reduced affinity of these P450s for carbon monoxide. Plant CYP74s have been classified in subfamilies $A$ to $G$ based on sequence similarity. The two subfamilies present in Arabidopsis ( $A$ and $B$ ), which seem to be present in most, if not all, plant species, each have a single member in the Arabidopsis genome: CYP74A1 and CYP74B2.

Plant CYP74 A and B subfamilies use the same substrates (fatty acid hydroperoxides generated by lipoxygenases) but form different products (Figure 14). The CYP74A subfamily converts C18 hydroperoxides to unstable C18 epoxides (allene epoxides). They are thus allene oxide synthases (AOS) and catalyze the first committed step in the biosynthesis of the octadecanoid pathway, which generates signaling molecules essential for host immunity and plant development such as jasmonates and their active precursor 12-oxo phytodienoic acid (12-OPDA). Being oxygenated derivatives of polyunsaturated fatty acids, plant octadecanoids share structural and functional properties with animal prostaglandins.

Expression of CYP74A1 is predominantly found in leaves and flowers of mature $A$. thaliana plants (Laudert and Weiler, 1998). The single and intronless gene was isolated from Arabidopsis, and its expression studied using promoter:GUS translational fusions (Kubigsteltig et al., 1999). Developmental control of CYP74A includes expression confined to veins in

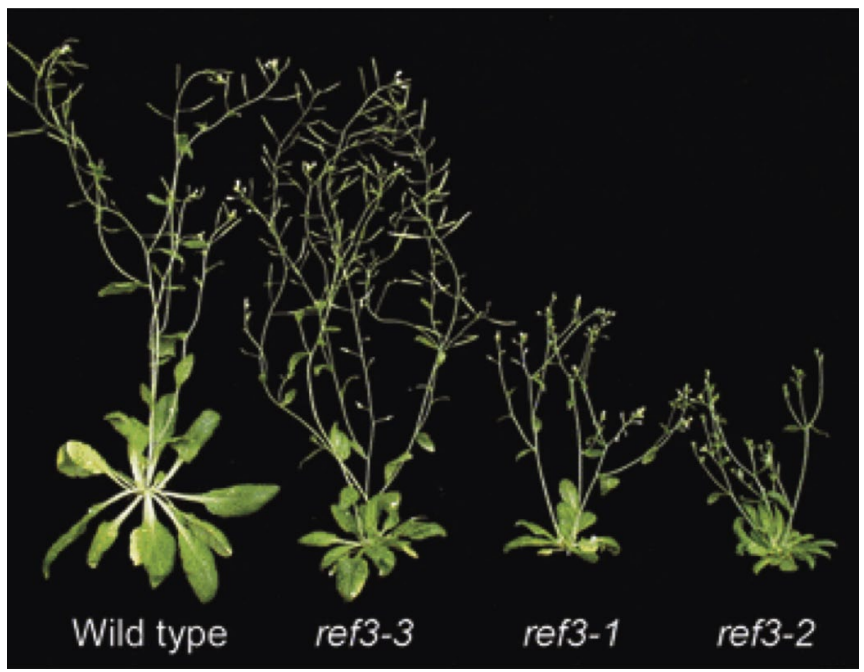

Figure 13. Developmental abnormalities in the ref3 mutants.

Plant with strong ref-1 and ref-2 alleles are dwarfed and show changes in apical dominance. Image reprinted from Schillmiller et al. (2009) with permission from Wiley-Blackwell Publishing. 


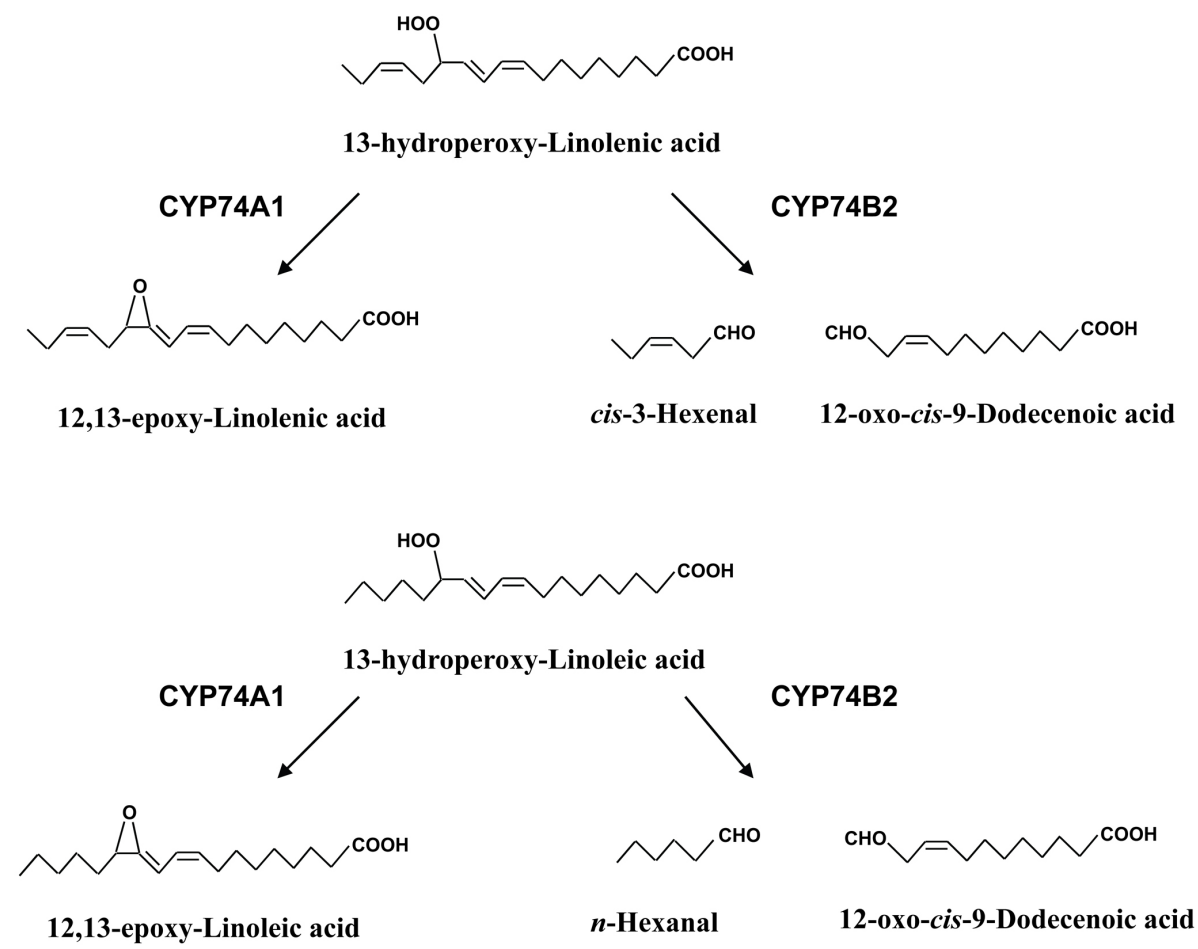

Figure 14. Reactions catalyzed by CYP74s.

CYP74A is an allene oxide synthase (AOS) and dehydrates 13-hydroperoxides of both linoleic and linolenic acids into unstable C18 allene oxides.

CYP74B2 catalyzes the cleavage of 13S-hydroperoxy-9(Z),11(E),15(Z)-octadecatrienoic acid (linolenic acid 13-hydroperoxide) 10 times more efficiently than that of 13S-hydroperoxy-9(Z),11(E)-octadecadienoic acid (linoleic acid 13-hydroperoxide).

young leaves that spreads throughout leaves and cotyledons in older plants (Figure 15). Transient expression is also observed in flower organs: weak and transient in developing carpels, very strong in maturing pollen and at the basis of anther filaments. In addition, rapid increases in CYP74A transcripts, protein, AOS catalytic activity and jasmonic acid are induced in wounded leaves (Laudert et al., 2000; Laudert and Weiler, 1998). Similar increases, although smaller and delayed, are also observed in the intact leaves of wounded plants. Stress, salycilate and ethylene-responsive elements are found in the CYP74A promoter which drive wound-, jasmonate-, 12-OPDA- and coronatine-induced expression of GUS. An interesting topological analysis of this induction can be found in Kubigsteltig et al. (1999). The most striking aspect of CYP74A regulation is its feedback amplification by downstream metabolites that leads to an increased output of the jasmonate pathway capacity.

Direct evidence for the biological roles of CYP74A1 came from the analysis of the T-DNA insertional knock-out mutant aos (Park et al., 2002). Kinetics of jasmonate accumulation induced by wounding showed that jasmonate biosynthesis was completely blocked in this mutant. The phenotype of the aos mutant consisted mainly of blocked anther development and small siliques producing no seeds. Male sterility was rescued to wild type by application of OPDA or a methyl ester of jasmonate, or by over-expression of CYP74A1. Interestingly, the gene expression level of the wound-induced lipoxygenase AtLOX2 (which acts upstream of AOS in the pathway) was not affected by wounding in the aos knock-out mutant. This result indicated that in aos plants the transduction of wound signals to AtLOX2 was interrupted or not generated because of the lack of jasmonates. Analysis of transgenic Arabidopsis plants expressing AOS constitutively suggested that in wild-type plants CYP74A1 activity was limiting the level of jasmonates produced upon wounding, but not the basal jasmonate level present in unwounded plants. The transposoninduced mutation dde2-2, which was identified based on fertility defects, has also been shown to be defective in CYP74A1 (Von Malek et al., 2002). Male sterility could be rescued by methyl jasmonate application and over-expression of CYP74A1 coding sequence as for the aos mutant. Recent observations on the dde2-2 mutant show that CYP74A1 and its octadecanoid products are involved in sensitivity of leaves and seedlings to osmotic (sorbitol) stress (Seltmann et al., 2010).

The catalytic activity of AOS has been extensively studied in Arabidopsis. Detergent-solubilized recombinant CYP74A1 expressed in $E$. coli was shown to catalyze the dehydration of both 13-hydroperoxylinoleic acid (13S-hydroperoxy-9(Z),11(E)-octadecadienoate) and 13-hydroperoxylinolenic acid (13S-hydroperoxy-9(Z),11(E),15(Z)-octadecatrienoate) into allene oxides (Laudert et al., 1996). It was later demonstrated that detergent-free recombinant CYP74A1, which was found to be a highly water-soluble monomeric protein, was also active on 9-hydroperoxylinoleic and 9-hydroperoxylinolenic acids generated by 9-lipoxygenases and thus had a dual 9/13-AOS specificity (Hughes et al., 2006). Addition of detergent micelles, but not detergent monomers, caused 


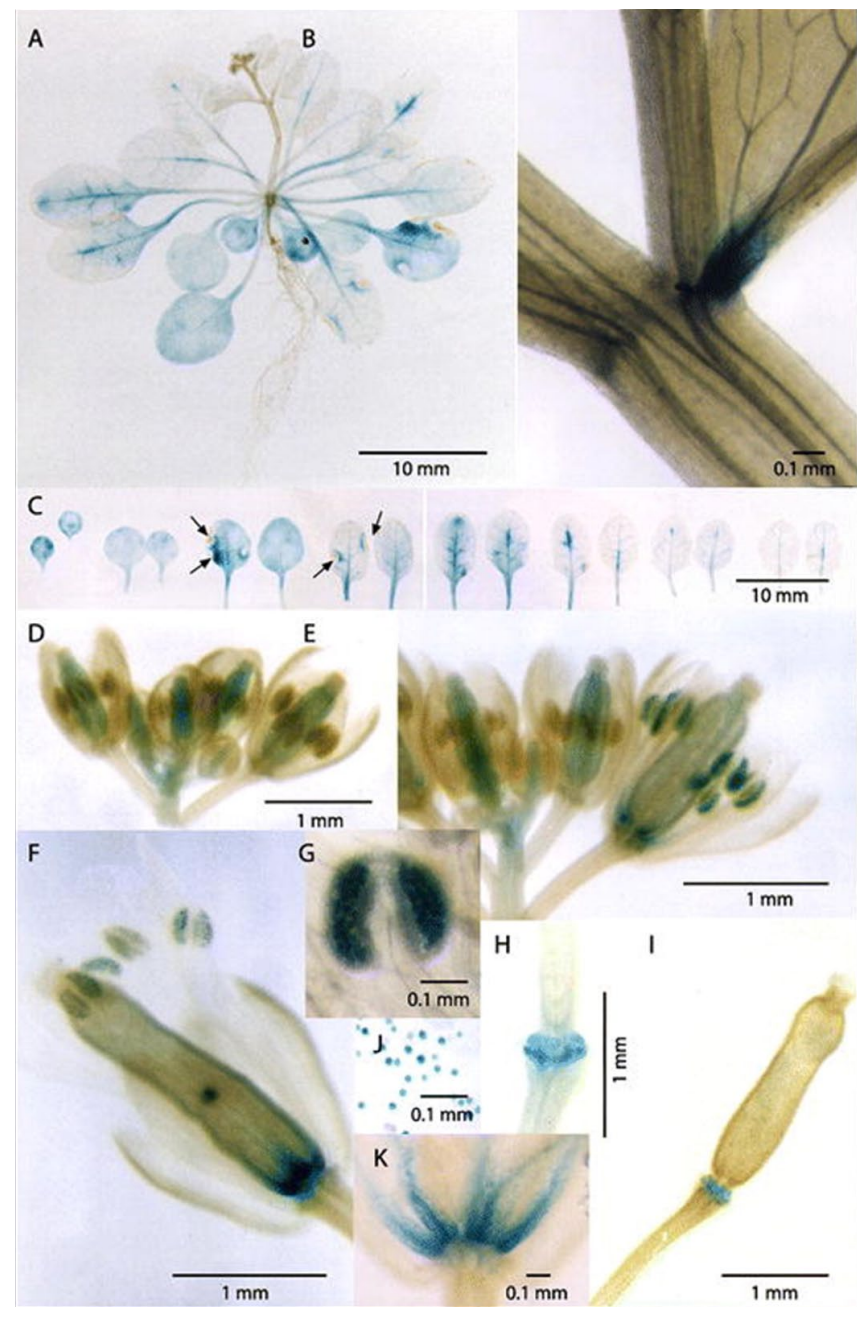

Figure 15. Developmental control of CYP74A.

Arabidopsis thaliana (A-I, K) or Nicotiana tabacum (J) plants grown under sterile conditions and harboring the translational CYP74A promoter:uidA fusion.

(A) Overview of a 3-week old plant. (B) Leaf base and stipule of a bract of the inflorescence axis. (C) Detached leaves of the plant shown in A arranged in the order of development (from left to right: cotyledons to younger leaves), necrotic areas marked by arrows. (D)(E) Developing flower buds. (F) Flower at the stage of fertilization. (G) Close-up of anther. (H)(I) Abscission zone scars after shedding of flower organs. (J) Pollen grains of transgenic tobacco. (K) Close-up of the bases of floral organs. Image reprinted from Kubigsteltig et al. (1999) with permission from Springer.

a drastic increase in AOS activities and greatly favored 13-AOS specificity. Activation of CYP74A1 observed in vitro upon binding of detergent micelles may bear relevance to the in vivo regulation of AOS activity following binding to cell membranes. CYP74A1 has been detected in isolated chloroplast envelope membranes (Ferro et al., 2010) and plastoglobules (Vidi et al., 2006).

The crystal structures of CYP74A1, free or in complexes with analogs of substrates or reaction intermediates, have been solved (Lee et al., 2008). This is the first three-dimensional structure of a plant cytochrome P450 (Figure 16). CYP74A1 lacks the

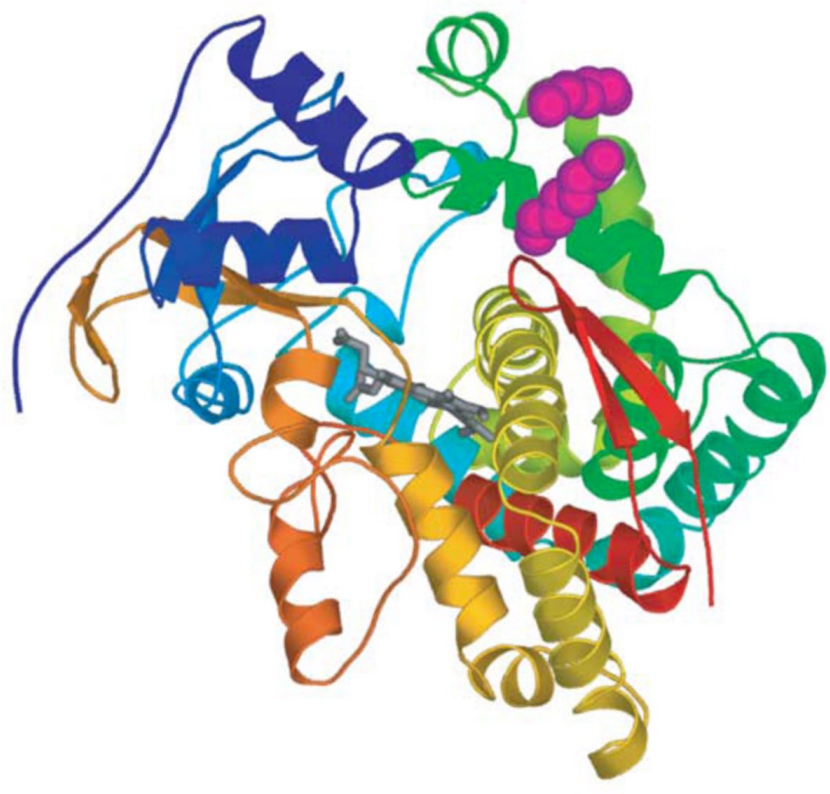

Figure 16. Ribbon diagram of CYP74A1 crystal structure.

$\mathrm{N}$-terminus is in blue and C-terminus in red. Hydrophobic tails of two detergent molecules interacting with the membrane-associated nonpolar surface are represented in magenta. Image reprinted from Lee et al. (2008) with permission from Nature Publishing Group.

$\mathrm{N}$-terminal transmembrane domain found in canonical plant or mammalian P450s but has a membrane-binding loop similar to mammalian CYP2C5. Several major structural features explained the absence of monooxygenase activity in CYP74A1. Among these, the most striking was perhaps the insertion of nine residues into the middle of the heme-binding loop. This modification is completely specific to CYP74 sequences and conserved in all of them. CYP74A1 structure and site directed mutagenesis experiments performed in the same study also revealed that CYP74A1 has an unusual active site with a Phe residue essential to control the reactivity of the epoxyallylic radical and its carbocation. Mutation of this Phe residue to Leu decreased AOS activity and allowed apparition of robust hydroperoxide lyase activity.

CYP74s from the B subfamily are hydroperoxide lyases (HPL) that cleave $\mathrm{C} 18$ hydroperoxides into $\mathrm{C} 6$ aldehydes and $\mathrm{C} 12$ $\omega$-oxo fatty acids (Figure 14b). C12 products lead to the formation of traumatin implicated in wound signaling, while C6 aldehydes are the basis of the "green note" flavor from disrupted plant tissues and an important determinant of fresh fruit and vegetable flavor. The described impacts of the C6 green leaf volatiles include antimicrobial effects, induction of phytoalexin accumulation, and inhibition of seed germination (Bate et al., 1998).

The first CYP74B cDNA was isolated from pepper bell, but the Arabidopsis cDNA was also isolated (Bate et al., 1998). The protein expressed in $E$. coli was shown to catalyze the cleavage of 13S-hydroperoxy-9(Z),11(E),15(Z)-octadecatrienoic acid 10 times more efficiently than that of $13 S$-hydroperoxy- $9(Z), 11(E)$ octadecadienoic acid. 
The CYP74B2 gene is highly expressed in flowers, siliques and roots (Bate et al., 1998). Levels of transcripts detected in green leaves are low, but their accumulation is induced within 30 minutes after wounding. Plant treatment with $10 \mu \mathrm{M}$ methyljasmonate induces CYP74A expression but has no effect on the expression of $C Y P 74 B 2$.

While transcripts of CYP74B2 encode a full-length protein in Landsberg erecta (Ler) and accumulate at normal levels in the Columbia 0 ( $\mathrm{Col} 0$ ) ecotype, they have a 10 nucleotide deletion in the first exon (resulting in a truncated protein) and show reduced transcript levels (Duan et al., 2005). The CYP74B2 gene is thus not functional in Col 0 . Consistently, no HPL activity could be detected in the Col 0 ecotype and trans-2-hexenal C6 volatile products were absent. Therefore, CYP74B2 seems dispensable for normal growth and development and does not appear to be replaced by another enzyme.

\section{CYP75}

A single gene belonging to the CYP75 family is found in the Arabidopsis genome. CYP75B1 maps at the top of chromosome $\mathrm{V}$ and corresponds to the $t t 7$ mutation. The $t t 7$ mutants have a transparent testa phenotype, pale brown seeds due to the lack of tannins in the seed coats, reduced anthocyanins in the whole plant, and accumulate 4'-hydroxylated flavonoids, pelargonidin and kaempferol instead of the 3',4'-hydroxylated flavonoids which are present in the wild plants (Koornneef, 1982; Sheahan, 1993). Unusual di- and tri-glycosylated kaempferol derivatives have in particular been detected in the seeds of $t t 7$ plants (Kerhoas et al., 2006). It was thus assumed that the TT7 (or AtF3'H) gene encoded a flavonoid 3'-hydroxylase. This assumption was confirmed by the expression of the cDNA in yeast and the demonstration that recombinant CYP75B1 catalyzes the 3'-hydroxylation of the B-ring of flavonoids such as naringenin and dihydrokaempferol (Figure 17) (Schoenbohm et al., 2000). Sequencing of the first $t t 7-1$ allele then showed that the mutation leads to premature protein termination after 113 amino acids (Schoenbohm et al., 2000). Comparison of the $\mathrm{Col}$ and Ler sequences also revealed the presence of a $B s t X I$ restriction polymorphism. Several other tt7 alleles have been subsequently characterized. Among them the T-DNA insertion line tt7-4 that was used for more extensive analysis of the flavonoid profile showing increases in kaempferol

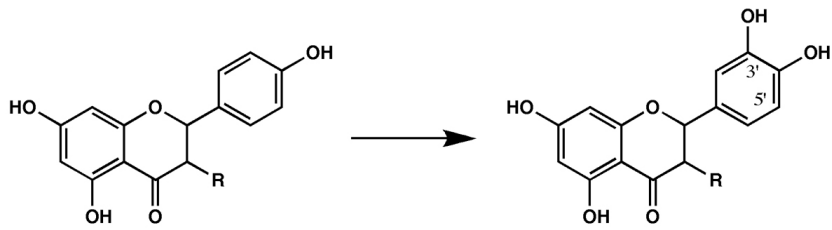

$\mathbf{R}=\mathbf{H}, \quad$ Naringenin $\mathbf{R}=\mathbf{O H}$, Dihydrokaempferol

$\mathbf{R}=\mathbf{H}, \quad$ Eriodictyol $\mathbf{R}=\mathbf{O H}$, Dihydroquercetin

Figure 17. CYP75B1 is a flavonoid 3'-hydroxylase.

CYP75B1 catalyzes the 3'-hydroxylation of the ring $B$ of naringenin and dihydrokaempferol to form eriodictyol and dihydroquercetin, respectively. aglycone and rhamnoside-glycoside (Routaboul et al., 2006). This study also detected a decrease in proanthocyanidins that were essentially formed of 4'-OH units corresponding to pelargonidins. A screen for T-DNA insertion lines to identify mutants defective in the synthesis of proanthocyanidins identified an allele of $t t 7$ (tt7-3) with a spotted pattern of proanthocyanidin deposition (Figure 18) in the seed coat producing only $8 \%$ of the wild-type level of condensed tannin as propelargonidin (Abrahams et al., 2002). The latter appeared as discrete spots within the vacuole of the endothelial cells. Probably as a result of a weaker testa, a $t t 7$ frameshift mutant allele (ctg225) was recovered in screens for mutants germinating faster at $10^{\circ} \mathrm{C}$ (Salaita et al., 2005). More surprisingly, one other $t t 7$ insertion allele (tt7-4 sib) was selected as a high temperature germination (thermoinhibition)-resistant mutant (Tamura et al., 2006). A model for the CYP75B1 active site architecture and substrate anchoring has been proposed by Rupasinghe et al. (2003).

CYP75B1 is highly expressed in siliques, flowers and senescent leaves, in agreement with the distribution of 3',4'-hydroxylated flavonoids in the $A$. thaliana plant (Routaboul et al., 2006). Transcripts also accumulate after UV light treatment of cultured cells which accumulate 3',4'-hydroxylated flavonoids (quercetin conjugates) in the vacuole. The $t t 7$ mutants, which are less tolerant than wild-type plants to UVB radiation, were shown to accumulate only kaempferol glycosides instead of quercetin conjugates upon UVB treatment (Ryan et al., 2001). Surprisingly, MYB12 that regulates a large subset of genes in the flavonoid pathway, such as chalcone synthase, chalcone isomerase, flavanonone 3-hydroxylase and flavonol synthase, does not influence the expression of CYP75B1 (Mehrtens et al., 2005). CYP75B1 thus seems to be regulated independently from most major genes in the pathway.

Cytochrome P450 enzymes are proposed to serve as nucleation sites for the formation of metabolons. To investigate if CYP75B1 could serve as membrane anchor for a flavonoid metabolon, the sites of accumulation of chalcone synthase and chalcone isomerase have been investigated in the root of the $t t 7$ mutant (Saslowsky and Winkel-Shirley, 2001). Their levels of accumu-
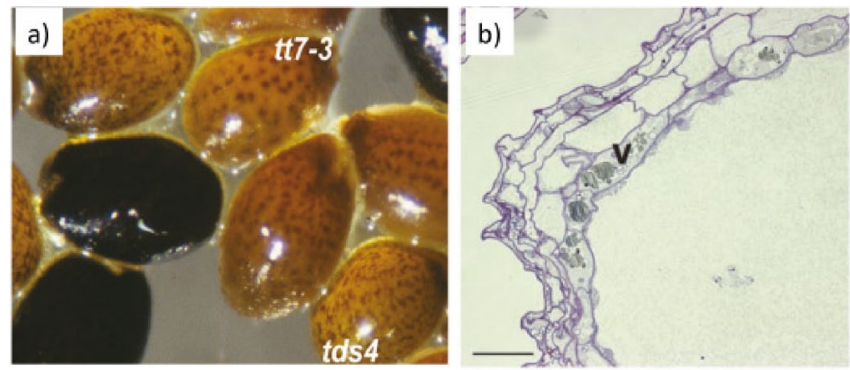

Figure 18. Proanthocyanidin deposition in the seed coat of the $t t 7-3 \mathrm{mu}-$ tant. Reprinted from Abraham et al. (2002).

(A) The tt7-3 mutants defective for CYP75B1 show a characteristic spotted pattern deposition of proanthocyanidin upon staining with $p$-dimethylaminocinnamaldehyde.

(B) Microscopic section of developing seeds at the heart stage showing small round inclusions of proanthocyanidin in the vacuole. 
lation appeared modified in root cortex and epidermal cells and association with electron-dense regions were not observed in the mutant compared to wild type. However, localization of both enzymes to the ER and tonoplast was not affected, suggesting that CYP75B1 is not critical for their membrane association.

The CYP75A subfamily that catalyzes both the flavonoid 3' and 5'-hydroxylations initially reported in petunia (Holton et al., 1993) is not represented in Arabidopsis.

\section{CYP76}

There are nine CYP76 genes in the Arabidopsis genome, eight belong to the CYP76C subfamily (including one pseudogene), a single one to the subfamily CYP76G. The function of these P450s is not known, except for a patent claiming geraniol 10-hydroxylase activity for CYP76C1 (Otah and Mizutani, 1998). A role in isoprenoid metabolism is supported by the functions reported for CYP76B6 in Catharanthus roseus (geraniol hydroxylase: Collu et al., 2001) and for CYP76M7 in rice (ent-cassadiene hydroxylase: Swaminathan et al., 2009). CYP76C1 to C4 form a small cluster on chromosome 2, and appear to have arisen by gene duplication. The expression of two genes in the cluster has been compared to that of other Arabidopsis P450s in two independent studies (Godiard et al., 1998; Mizutani and Ohta, 1998). CYP76C1 was shown to be highly expressed in flowers, and at lower levels in stems, siliques and leaves (Mizutani et al., 1998). Its expression is decreased by wounding. It gradually increases upon illumination and drops when plants are returned to the dark. CYP76C2 expression was associated with stress response, being very low in young tissues, but induced during hypersensitive response to pathogens, senescence, after wounding, heat-shock, or lead nitrate treatment (Godiard et al., 1998).

\section{CYP77}

Members of this family belonging to the CYP71 clan are first found in Lycopods (Nelson and Werck-Reichhart, 2011). Most CYP77s contain no intron. Five CYP77As (CYP77A4, CYP77A6, CYP77A7, CYP77A9, pseudogene CYP77A5) and one CYP77B (CYP77B1) are present in the Arabidopsis genome. CYP77A4 and CYP77A6 were independently identified as putative fatty acid oxidases, based on gene co-expression profiles with a fatty acid hydroxylase and other lipid metabolism genes (Sauveplane et al., 2009), and with cutin acyltransferase genes, respectively (Li-Beisson et al., 2009). Heterologous expression of CYP77A4 in Saccharomyces cerevisiae showed that the enzyme converted linoleic acid (cis, cis-9,12-octadecadienoic acid) into 12,13-epoxyoctadeca-9-enoic acid (Figure 19) and then into the 9,10-12,13-diepoxide (Sauveplane et al., 2009). CYP77A4 was also able to catalyze the in vitro formation of the three mono-epoxides of $\alpha$-linolenic acid (cis, cis, cis-9,12-15-octadecatrienoic acid), previously described as antifungal compounds. It requires a double bond in the cis configuration to metabolize C18 fatty acids, since neither stearic acid (C18 saturated fatty acid) nor linolelaidic acid (trans,trans-9,12 octadecadienoic acid) can be used as substrates.

Together genetic and biochemical data indicate that CYP77A6 is the in-chain fatty acid hydroxylase forming the cutin monomer

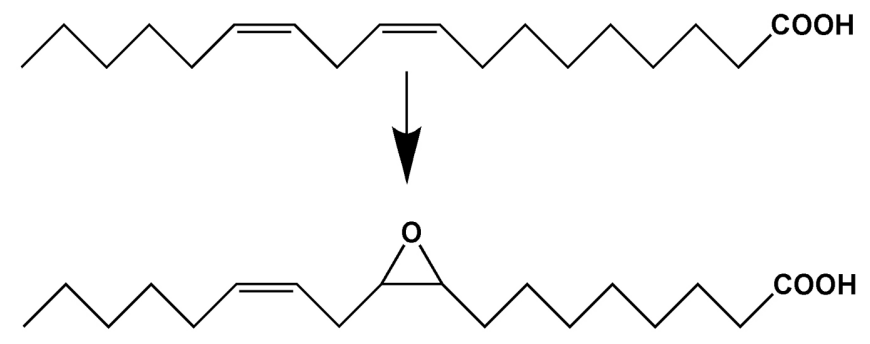

Figure 19. Conversion of linoleic acid into the corresponding 12,13-epoxy fatty acid.

The epoxy fatty acid product was formed in vitro by incubation of linoleic acid with NADPH and microsomes of yeast expressing recombinant $\mathrm{CY}$ P77A4.

10,16-dihydroxypalmitate and its 9,16 isomer (Li-Beisson et al., 2009). The 10,16-isomer is the major monomer in the cutin of Arabidopsis flowers and a major cutin monomer in other organs of many plant species. Insertional knock-outs cyp77a6-1 and cyp77a6-2 were isolated and shown to have a strong reduction in cutin content, with no detectable 10(9),16-dihydroxypalmitate. Comparison of cutin monomer profiles in knock-outs for CYP77A6 and the fatty acid $\omega$-hydroxylase CYP86A4 provided genetic evidence that CYP77A6 is an in-chain hydroxylase acting subsequently to CYP86A4 in the synthesis of 10,16-dihydroxypalmitate. In-chain hydroxylase activity of CYP77A6 was demonstrated by production of 10(9)(8),16-dihydroxypalmitate from 16-hydroxypalmitate using CYP77A6-expressing yeast microsomes. These results thus identify in Arabidopsis the in-chain hydroxylase of the biosynthesis pathway of $\mathrm{C} 16$ cutin monomers, a P450-type enzyme that was characterized biochemically several decades ago in broad bean (Soliday and Kolattukudy, 1978), but whose gene had not been identified. Strikingly, petals and sepals of CYP77A6 knock-outs had no cuticle and no surface nanoridges as shown by electron microscopy (Figure 20). Therefore, the formation of these distinctive surface nanostructures of flowers, which are thought to help attract pollinators, requires active synthesis of the cutin polymer and the structures are not caused by undulations of the cell wall that are then simply covered by the cutin layer.

The disruption of CYP77A7 in the Ds insertion mutant pgd3 causes a defect in pollen germination (Boavida et al., 2009). In this mutant, all pollen grains are viable, but pollen tube initiation and growth occur in only about $50 \%$ of them (probably the wildtype pollen grains in the tetrad). The defect is not male gametophyte-specific, but mutants have also a female transmission defect. The biochemical function of CYP77A7 is so far unknown.

\section{CYP78}

The six Arabidopsis CYP78 genes are scattered over the whole genome. Like CYP78 members from other plant species, they all belong to the same CYP78A subfamily. The catalytic function of the Arabidopsis CYP78 proteins is so far not known. The pattern of expression and in vivo impact of the related genes suggest their possible involvement in the biosynthesis of some unknown 


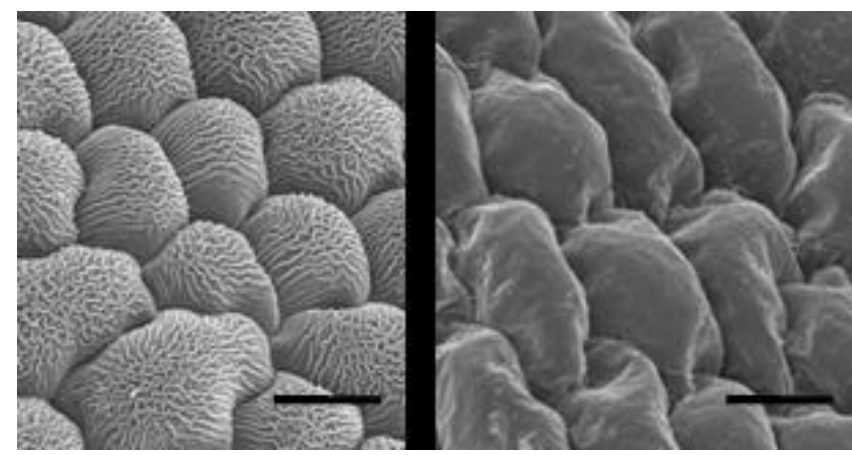

Figure 20. Abaxial epidermal cells of petals in WT and ccyp77a6-1 knockout mutants as seen by scanning electron microscopy.

Surface nanoridges are absent in the mutant. Left: wild-type; right: $c y$ p77a6-1 mutant. Abaxial sides of petals are shown. The same ridgeless phenotype was observed on adaxial sides, in sepals and in the cyp77a6-2 mutant. Scale bars: $10 \mu \mathrm{m}$. Reprinted from Li-Beisson et al. (2009) with permission from National Academy of Sciences.

type of plant growth regulator (Ito and Meyerowitz, 2000; Zondlo and Irish, 1999). Homozygous insertional mutants of CYP78A6, which is ubiquitously expressed in all plant tissues, showed no mutant phenotype (Ito and Meyerowitz, 2000). The other CYP78 genes have been described as flower- or meristem-specific in their expression.

CYP78A5 was isolated from a differential screening for genes expressed in early flower development (Zondlo and Irish, 1999). In situ hybridization showed that it is strongly expressed in the peripheral region of the vegetative and reproductive shoot apical meristems, defining a boundary between the central meristematic zone and the developing organ primordia. Expression was detected throughout floral development, in the organ primordia, on the adaxial side of the pedicels and floral organs, and in the developing ovules. Constitutive over-expression of the gene results in plants with twisted and kinked stems with an irregular surface. Flowers are very slow to open, with stunted petals and stamens, and disorganized tissues. They lead to very few viable seeds. Cell morphology is altered with abnormal expansion of epidermal tissues, defects in cell size and shape in internal tissues. The most striking defects are observed in tissues that undergo a directional cell elongation. Accordingly, it was proposed that CYP78A5 is involved in regulating directional cell growth.

Three CYP78A5 mutants termed kluh (klu) have been isolated from an EMS-mutagenized population screened for alterations in floral organ size (Anastasiou et al., 2007) (Figure 21). In addition, an insertional knock-out line for CYP78A5 showed a higher rate of leaf initiation (Wang et al., 2008) (Figure 22). The smaller size of leaves, sepals, and petals in homozygous klu plants was found to be due to a lower number of cells and not to smaller cells (Anastasiou et al., 2007). Over-expression of CYP78A5 indicated that this protein promotes growth by cell proliferation with final organ size depending on its level of expression. Use of klu plants in which CYP78A5 expression can be induced showed that CYP78A5 prevents a premature arrest of growth by maintaining cell proliferation. Comparison of regions of organ proliferation and CYP78A5 petal expression domains (Figure 23) indicated further that CYP78A5 acts non-cell-autonomously (Anastasiou et al., 2007). The same mode of action occurs in seeds. KLU is expressed in the inner integument of developing ovules and stimulates cell proliferation, thereby determining seed size (Adamski et al., 2009). Because the CYP78A5 protein itself was localized to the ER and did not appear to move to the regions of cell proliferation, it was concluded that CYP78A5 generates a mobile growth-promoting signal (Anastasiou et al., 2007). The CYP78A5-dependent signal has been shown to move beyond individual floral organs, allowing coordination of their growth and ensuring floral symmetry (Eriksson et al., 2010).

The facts that the transcriptional response to $K L U$ activity is found to be distinct from that of classical phytohormones, and that no genetic interaction can be evidenced between $K L U$ and other genes known to regulate organ growth, support the view that CYP78A5 belongs to a novel signaling pathway regulating organ size (Anastasiou et al., 2007). Given that maize CYP78A1 shows in vitro $\omega$-hydroxylation activity on lauric acid (Imaishi et al., 2000), it has been proposed that the mobile growth signal produced by CYP78A5 is a fatty acid-related molecule.

A loss-of-function mutant for CYP78A7 has also been isolated (Wang et al., 2008). It appears phenotypically normal. However, embryos of the double knock-out mutant cyp78a5 cyp78a7 do not increase much in size and have abnormal shoot apical meri-

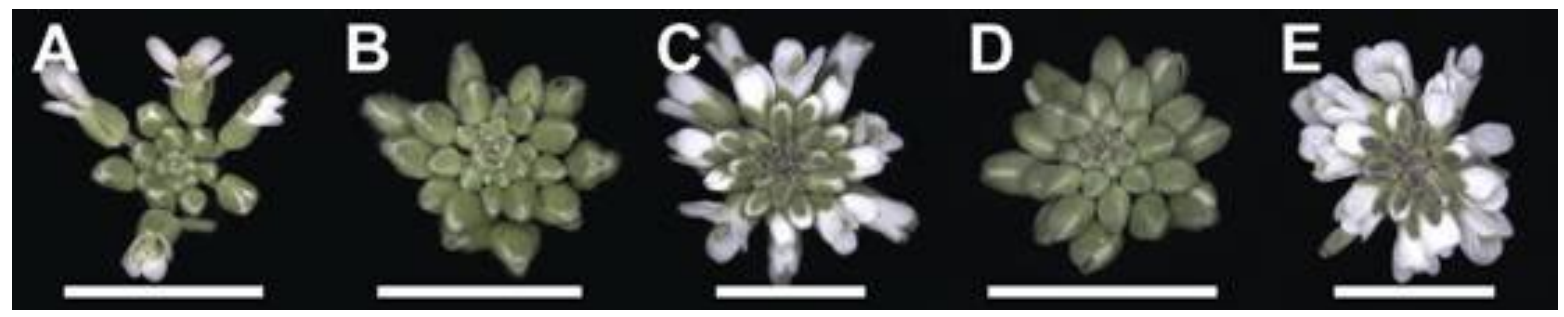

Figure 21. Phenotype of inflorescences in lines under- or over-expressing CYP78A5.

(A-E) Inflorescences of (A) Ler control, (B) klu-2, (C) the KLU-overexpressing line klu-2, RLox, (D) Col-0 wild-type, and (E) klu-4. Scale bars are 8 mm. Reprinted from Anastasiou et al. (2007) with permission from Elsevier. 
stems that initiate supernumerary cotyledons (Figure 22). When seedlings survive, they give rise to small plants with compacted rosette leaves, which produce no seeds. It seems therefore that CYP78A5 and CYP78A7 play redundant roles in the regulation of the growth of the shoot apical meristem and the rest of the plant.

CYP78A9 was isolated from an activation tagging line showing large seedless fruits and altered cell growth (Ito and Meyerowitz, 2000). The mutant phenotype initially characterized in the apetala2-1 background, was slightly altered in a wild-type background, with fruits more elongated than wide (Figure 24). Over-expression of CYP78A9 allows fruit growth independently of fertilization. Flower modifications are similar to those observed in CYP78A5 overexpressors, with shortened stamens and reduced aborted ovules, which suggests that both genes may be involved in the same pathway. CYP78A9 however differs from CYP78A5 by its very strict tissue-specific expression in funiculi
A
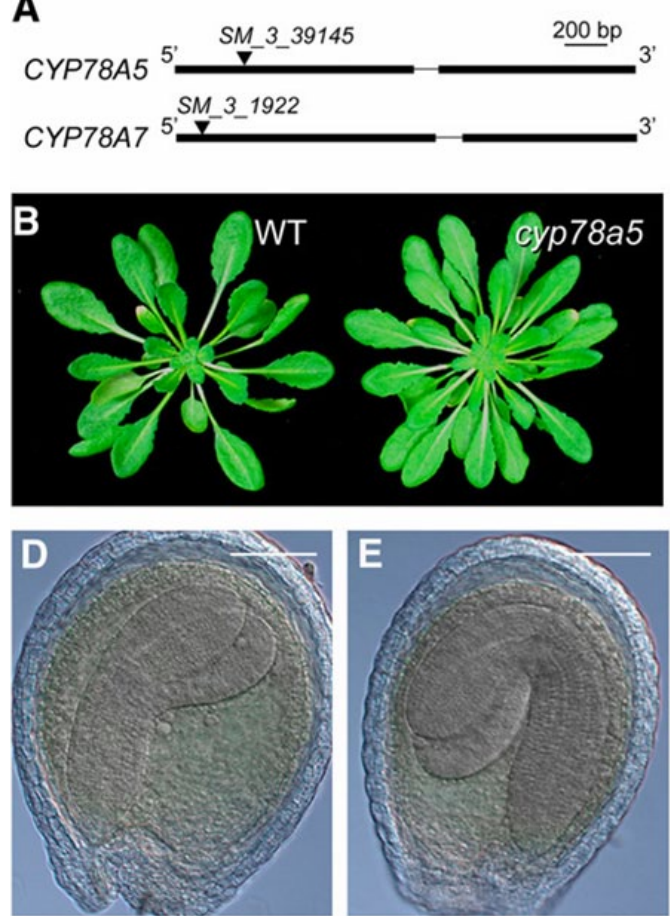

C
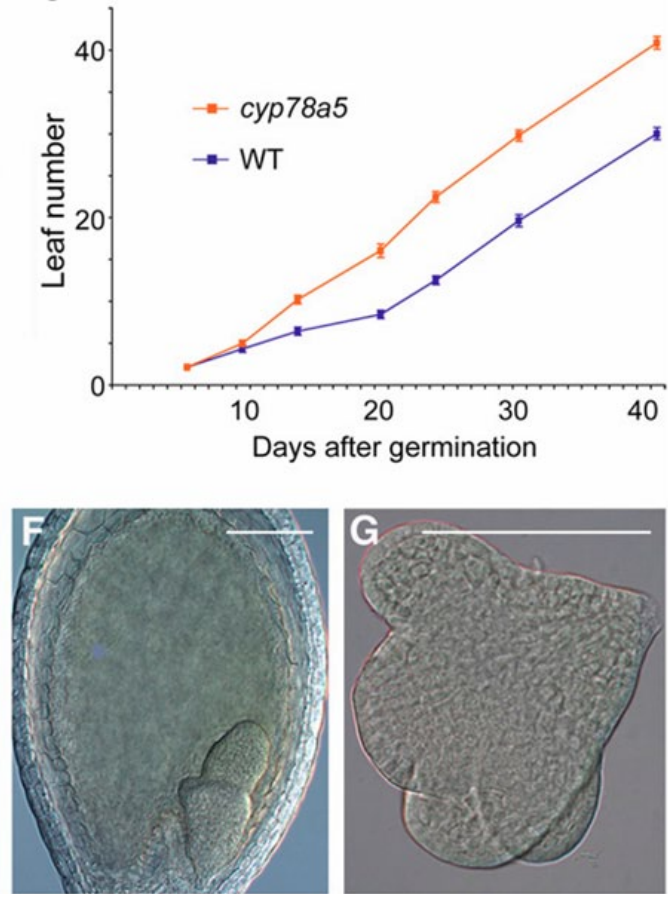

Figure 22. Phenotype of cyp78a5 and cyp78a7 insertional mutants.

(A) T-DNA insertion sites in CYP78A5 and CYP78A7. (B) Rosettes of 30-d-old plants grown in short days. (C) Number of leaves in short-day-grown plants. (D) and (E) Wild-type embryos at bent-cotyledon stage (D) and at maturity (E). (F) and (G) cyp78a5 cyp78a7 embryos at bent-cotyledon stage (F) and at maturity (G). Reprinted from Wang et al. (2008).

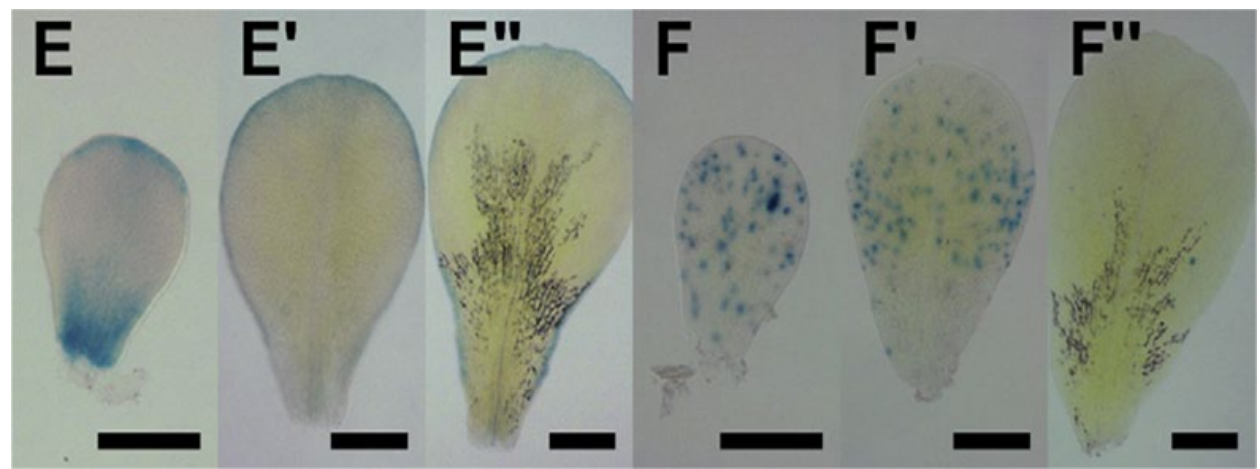

Figure 23. Expression of CYP78A5 compared to cell activity regions in developing petals.

pKLU/GUS expression pattern (E to E") does not occurr in regions of cell division activity (F to F"), which is monitored by the pAtCycB1;1/CDBGUS reporter. Petals in (E) and (F), in ( $\left.\mathbf{E}^{\prime}\right)$ and (F'), and in ( $\left.\mathbf{E}^{\prime \prime}\right)$ and (F') are from flowers in comparable developmental stages. Scale bar $100 \mu \mathrm{m}$. Reprinted from Anastasiou et al. (2007) with permission from Elsevier. 

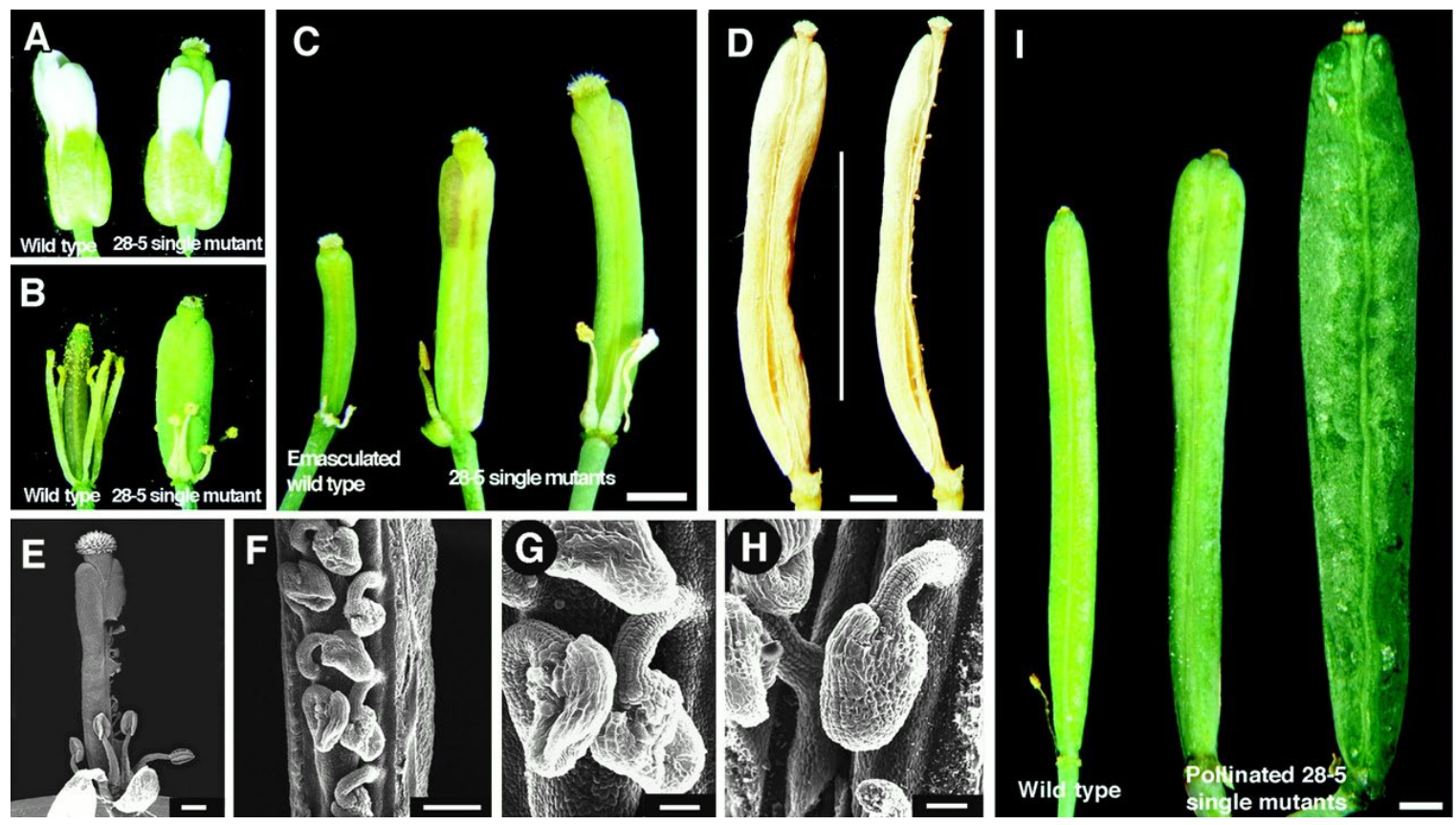

Figure 24. Phenotype resulting from the over-expression of CYP78A9. Reprinted from Ito and Meyerowitz (2000).

(A) and (B) Wild-type (left) and mutant flowers (right). The length of the sepals and petals of mutant flowers did not differ from wild-type. However stamens of mutants were approx. 50\% shorter than those of the wild type. The single mutant pistils were longer and wider than those of the wild type.

(C) Wild type siliques 14 days after emasculation (left), mutant siliques 3 days (middle), and 5 days (right) after anthesis. The siliques of mutants continued to elongate without fertilization. Stigmatic papillae of elongating siliques were still intact.

(D) Unpollinated dried mutant silique. The silique of the mutant showed a parthenocarpic phenotype. One carpel was removed to view the inside (right). No seeds were produced.

(E) SEM of mutant pistil. Part of one carpel was removed to view the ovules inside.

$(F)$ to $(H)$ Close-up view of the ovules. Most of the ovules were shriveled around the region where the embryo sac would be in the wild type (G). However, a few ovules showed normal morphology $(\mathbf{H})$.

(I) Self-pollinated wild-type silique (left) and pollinated mutant silique with wild type pollen (middle). Pollinated silique elongated to as much as $18 \mathrm{~mm}$ in one extreme case (right).

(the stalks of the developing ovules) during late flower development (upon anthesis). It was proposed that one of the functions of CYP78A9 is to produce a signal that activates or enhances fruit development. Identifying the functions of the CYP78s will therefore bring an interesting break-through in the understanding of plant growth regulation.

\section{CYP79}

There are seven CYP79 genes and six pseudogenes in Arabidopsis covering four subfamilies. The presence of multiple CYP79 pseudogenes is indicative of the glucosinolate pathway having evolved relatively recently. CYP79s are multifunctional P450s catalyzing two consecutive $N$-hydroxylations of amino acids followed by a dehydration and decarboxylation reaction (Figure 25) (Halkier et al., 1995; Sibbesen et al., 1995). Except for CYP79C1, CYP79C2, and CYP79F2, whose functions are currently unknown, all the Arabidopsis CYP79s metabolize amino acids or their chain- elongated forms into the corresponding aldoximes in the biosynthesis of glucosinolates (Hull et al., 2000; Mikkelsen et al., 2000; Wittstock and Halkier, 2000). Glucosinolates are sulfur-containing secondary metabolites present in all crucifers and are synthesised from amino acids (Halkier and Gershenzon, 2006). Arabidopsis contains at least 24 different glucosinolates derived from tryptophan, homophenylalanine, and chain elongated homologs of methionine. The CYP79 homologs are highly substrate specific and are thought to dictate the specific glucosinolate profiles in a plant.

CYP79B2 and CYP79B3 catalyze the conversion of tryptophan to indole-3-acetaldoxime (Hull et al., 2000; Mikkelsen et al., 2000) (Figure 25). Indole-3-acetaldoxime is a shared intermediate between the biosynthesis of indole glucosinolates and the main growth regulator auxin indole-3-acetic acid (IAA). Accordingly, ectopic over-expression of CYP79B2 cDNA results in a significant overproduction of indole glucosinolates and in plants that exhibit a reduced growth phenotype (Mikkelsen et al., 2000). IAA levels were not measured in the CYP79B2 over-expression lines, but it is plausible that the observed phenotype can be associated with 


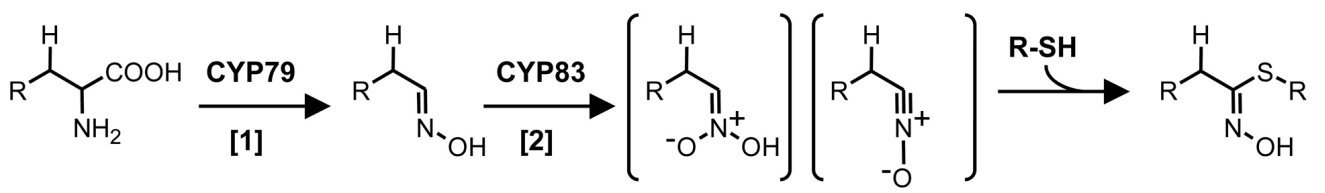

amino acid aci-nitro or nitrile oxide intermediate S-alkyl-thiohydroximates

\section{[1] CYP79A1:}<smiles>CCC(N)(Cc1ccc(O)cc1)C(=O)O</smiles>

tyrosine<smiles>O=C(O)C(O)(Cc1ccc(O)cc1)NO</smiles>

$N, N$-dihydroxytyrosine<smiles>O/N=C/Cc1ccc(O)cc1</smiles>

(E)-p-hydroxyphenyl acetaldehyde oxime

\section{Substrate \\ phenylalanine tryptophan}

mono- to hexahomomethionine penta- and hexahomomethionine
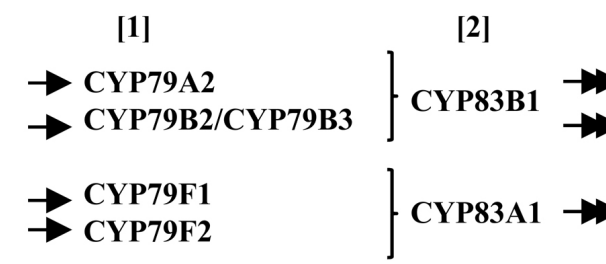

Product benzyl glucosinolates indole glucosinolates short- and long chain aliphatic glucosinolates

Figure 25. Role of CYP79s and CYP83s in the glucosinolate pathway.

CYP79 convert a range of aromatic or aliphatic amino acid precursors into the corresponding acetaldoximes. CYP83 catalyze the formation of aci-nitro or nitrile oxide intermediates, which in the presence of thiol compounds (R-SH) form S-alkyl-thiohydroximate adducts. In the absence of a thiol compound, the highly electrophilic product of CYP83 catalysis inactivates the enzyme.

an increase in free IAA. In a parallel study, over-expression of CYP79B2 did not alter the phenotype (Hull et al., 2000). However, neither indole glucosinolate levels nor IAA levels were measured in these transgenic lines, and the lack of a phenotype may be due to expression levels.

Recombinant CYP79A2 catalyzes the conversion of phenylalanine, but not homophenylalanine (Wittstock and Halkier, 2000). In good agreement with this, ectopic over-expression of CYP79A2 results in accumulation of benzyl glucosinolates (Wittstock and Halkier, 2000). Ectopic expression in Arabidopsis of CYP79D2 from the cyanogenic plant cassava (Manihot esculenta), which metabolizes valine and isoleucine to the corresponding oximes, resulted in accumulation of increased levels of the aliphatic glucosinolates methyl-, ethyl- and isobutyl-glucosinolates (Mikkelsen and Halkier, 2003). These lines showed enhanced resistance against the bacterial soft-rot pathogen Erwinia carotovora (Brader et al., 2006). Neither of the plants ectopically expressing Arabidopsis CYP79A2 nor cassava CYP79D2 showed any effect on diamondback moth preference and performance (Sarosh et al., 2010). A common feature of the glucosinolate and cyanogenic glucoside pathways is that a CYP79 catalyzes the first committed step from the precursor amino acid to the oxime in biosynthesis of both secondary metabolites. The glucosinolate pathway has most likely evolved by branching off from the ancient cyanogenic glucoside pathway and, accordingly, expression in Arabidopsis of CYP79s originating from cyanogenic plants results in accumulation of altered glucosinolate profiles (Bak et al., 2006).

CYP79F1 and CYP79F2 share 88\% amino acid sequence identity. Their genes are both located on chromosome 1, separated by less than 1600 bp. A knock-out of CYP79F1 by En-1 transposon tagging was designated the bus1-1 mutation. The bus1-1 mutants exhibit a short bushy phenotype with crinkled leaves and retarded vascularization (Figure 26). They are depleted in short chain methionine-derived glucosinolates, but not in longer chain methionine-derived glucosinolates (Reintanz et al., 2001). Auxin as well as indole glucosinolate levels are elevated in bus1-1. Similarly, co-suppression of CYP79F1 results in bushy plants with lowered levels of short-chain methionine-derived glucosinolates, increased expression of CYP79B2, and increased indole glucosinolates (Hansen et al., 2001). The Ds transposon null mutant supershoot $s p s$ is allelic to and phenotypically similar to bus1-1 (Tantikanjana et al., 2001). In the sps mutants, cytokinin and auxin levels are both elevated. This indicates the involvement of SPS/ CYP79F1 in hormone homeostasis. As expected, recombinant CYP79F1 metabolizes short-chained methionine analogs into the corresponding aldoximes precursors of glucosinolate biosynthesis (Hansen et al., 2001). This study demonstrates the pleotropic effects that can occur when studying metabolic mutants. 


\section{CYP80}

The CYP80 family is not represented in A. thaliana, but has been shown to be involved in phenolic coupling in biosynthesis of alkaloids in other plant species (see Kutchan, 1996). A CYP80 family member is found in papaya. The family is thus not lost in all Brassicaceae.

\section{CYP81}

There are eight CYP81Ds, four CYP81Fs, single CYP81H and $G$, and two CYP81Ks, with very diverse expression patterns and forming small clusters in the Arabidopsis genome. Only the function of CYP81F2 has been described so far. It was initially identified as one of the genes most strongly and rapidly induced upon
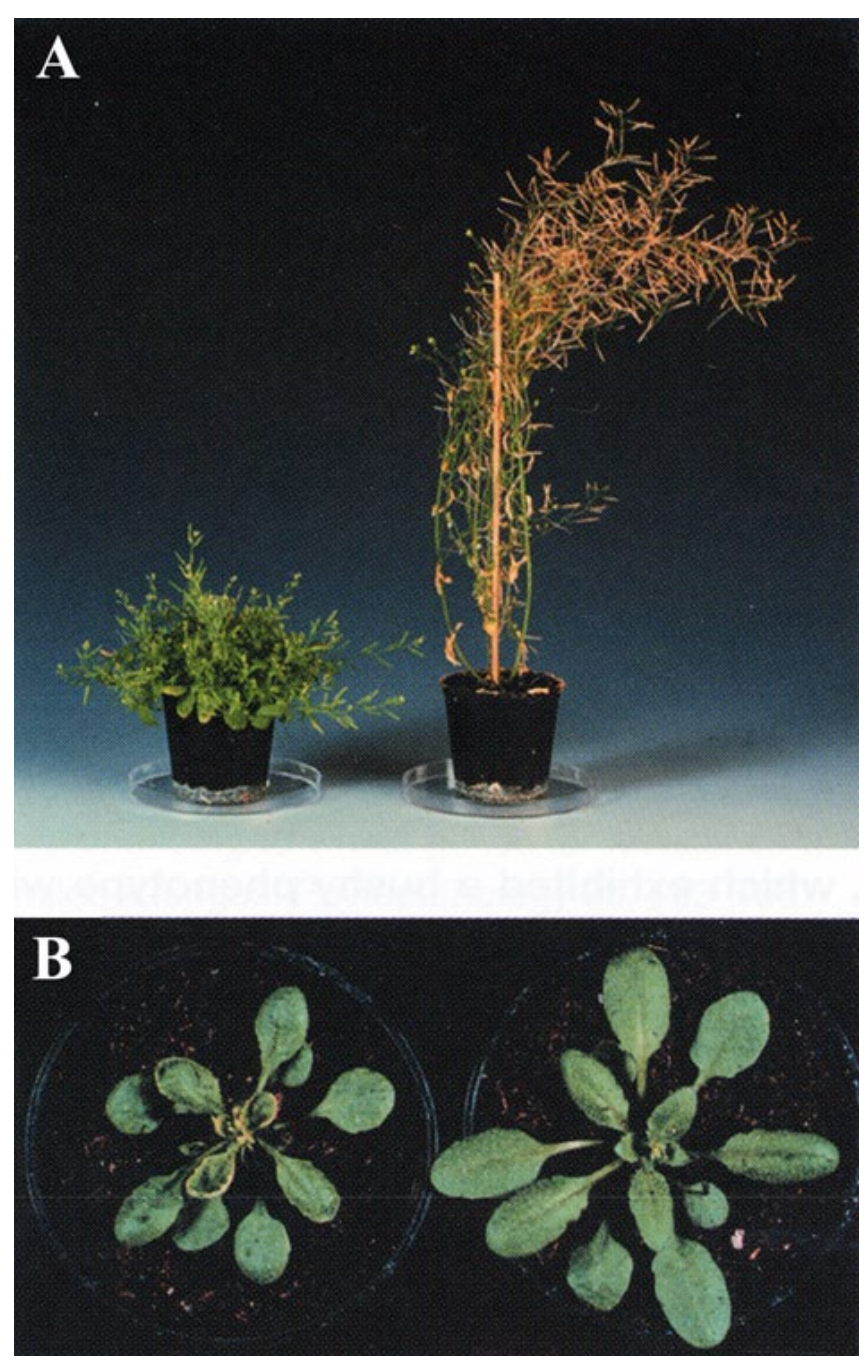

Figure 26. The bus1-1 phenotype induced by En-1 insertion in CYP79F1. Reprinted from Reintanz et al. (2001).

The mature bus1-1 mutant exhibits a small bushy phenotype. bus1-1 is shown on the left, wild type on the right. (B) Under normal greenhouse conditions, the bus1-1 mutant has smaller rosette leaves (3 weeks old). treatment with elicitors of defense responses such as oligogalacturonides and the bacterial flagellin peptide Flg22 (Galletti et al., 2008; Denoux et al., 2008), which suggested a role in the activation of defense responses. Using different and complementary approaches, three groups simultaneously provided evidence that CYP81F2 belongs to a pathway branching from the indole glucosinolates that are involved in different aspects of defense against microbial pathogens and insects (see CYP79s above). Metabolic profiling of mutants defective in their response to fungal pathogens led to the identification of 4-methoxyindole-3-ylmethylglucosinolate (4MI3G) as a mediator of broad-spectrum antifungal defense (Bednarek et al., 2009). This compound appeared to result from the combined activities of the myrosinase PEN2 and of CYP81F2 on indole glucosinolates as shown by the strong decrease in $4 \mathrm{MI} 3 \mathrm{G}$ in the cyp81f2 insertion mutants and their increased susceptibility to fungal pathogens. Dissection of the response to the bacterial elicitor Flg22 identified CYP81F2 and $4 \mathrm{MI} 3 \mathrm{G}$ as elements of the signaling cascade leading to callose deposition upon pathogen attack (Clay et al., 2009). The Flg22treated cyp81f2 insertion mutants showed reduced 4MI3G accumulation and increased susceptibility to Pseudomonas syringae PToDC3000. Suppressed callose deposition in the cyp81f2 and other callose deficient mutants was rescued with 4MI3G. The definitive proof that CYP81F2 is an indole-3-ylmethylglucosinolate 4-hydroxylase was provided by Pfalz et al. (2009) who, based on a combination of QTL and transcript profiling, associated CYP81F2 with the metabolic QTL Indole Glucosinolate Modifier 1 (IGM1) for the accumulation of 4-hydroxyindole-3-ylmethylglucosinolate and its methylated form $4 \mathrm{MI} 3 \mathrm{G}$. The catalytic function of CYP81F2 was confirmed by complementation of the metabolic phenotype of T-DNA-insertion mutants, and by expression of CYP81F2 in insect cells for the in vitro demonstration that the recombinant enzyme indeed catalyzes the 4-hydroxylation of the indole ring of indole-3-ylmethylglucosinolate (Figure 27). The IGM1 QTL is essentially due to variation in CYP81F2 expression, resulting from a combination of both cis- (through variation in the CYP81B2 promoter) and trans-acting expression QTL different

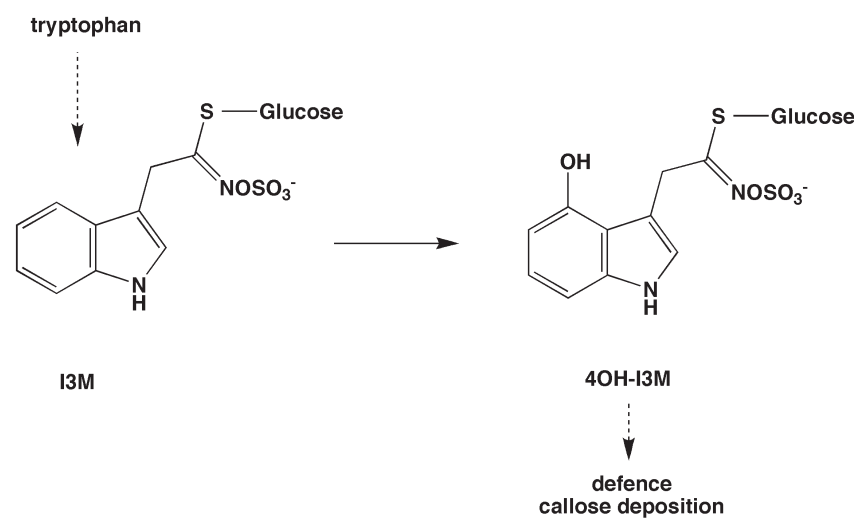

Figure 27. CYP81F2 catalyzes the conversion of the phytoanticipin indole-3yl-methyl glucosinolate (I3M) into 4-hydroxy-indole-3-yl-methyl glucosinolate $(4 \mathrm{OH}-\mathrm{I} 3 \mathrm{M})$ in the indole glucosinolate pathway to activate mechanisms of defense against pathogens and insects. 
from known regulators of indole glucosinolate synthesis. It had been previously reported that aphid feeding induced the production of $4 \mathrm{MI} 3 \mathrm{G}$ in $A$. thaliana (limited to the area in which the aphids were feeding), and that $4 \mathrm{MI} 3 \mathrm{G}$ added to an artificial diet caused a strong inhibition of aphid proliferation (Kim and Jander, 2007). This deterrent effect did not require the presence of myrosinase. Evaluation of insect performance on the cyp81f2 mutants confirmed that while expression of the gene does not affect herbivory by several generalist and specialists lepidopterans, it significantly contributes to plant resistance against green peach aphid, a generalist phloem-feeding herbivore (Pfalz et al., 2009; deVos and Jander, 2009). CYP81F2 might also have an adverse effect on the plant insect interaction. $4 \mathrm{MI} 3 \mathrm{G}$ was described as a very strong oviposition attractant for Plutella xylostella, suggesting that this compound might be involved in diamondback moth host recognition. Accordingly, reduced oviposition was observed on extracts from cyp81f2 plants compared to wild type. This was, however, not confirmed in whole plant experiments (Sun et al., 2009).

\section{CYP82}

The CYP82 family belongs to the CYP71 clan. It appeared early in Angiosperms and is first found in Magnoliids. Besides their expression patterns, limited information is available concerning the function of the cluster of the three CYP82Cs, and of the single CYP82F genes present in Arabidopsis. The function of the single CYP82G subfamily member has however been elucidated.

A large-scale approach screening for Arabidopsis P450s that could modify furanocoumarins spotted CYP82C2 and CYP82C4 as 8-methoxypsoralen hydroxylases (Kruse et al., 2008). This is however unlikely to be their function in the plant since furanocoumarins have never been detected in Arabidopsis. In agreement with this assumption, CYP $82 C 2$ was recently shown to be the gene affected by the jah1 mutation (T-DNA insertion) that leads to increased inhibition by jasmonic acid of root growth (Liu et al., 2010). The mutation also reduced JA-activated expression of pathogen defense-related genes and reduces plant resistance to Botrytis cinerea. A similar phenotype was reported for two additional T-DNA insertion mutants (SALK_128974 and SALK_024364) that were named jah1-2 and jah1-3. Conversely, over-expression mutants of CYP82C2 showed decreased sensitivity to JA in root growth assay, elevated response of plant defense genes and increased resistance to $B$. cinerea. RT-PCR analysis and promoter activity indicate that CYP82C2 is expressed predominantly in the roots and hypocotyls, mainly in vascular tissue. The gene is also expressed in the anthers during flower development, in young leaf trichomes and at the pedicel-silique junction (Figure 28). MeJA was reported to activate CYP82C2 promoter:GUS expression in the roots. Accumulation of transcripts in response to MeJA and Botrytis in soil-grown plants was detected at $24 \mathrm{~h}$ after treatment and was maximal after 2 days. MeJA induction of CYP82C2 occurred independently of COI1, but was negatively regulated by AtMYBC2. Investigations carried out to explore the metabolic impact of the jah1 mutation, demonstrated a substantial reduction of the JA-induced accumulation of tryptophan and indole glucosinolates, and of JA induction of their biosynthetic genes. Conversely, increased amounts of indole glucosinolates were observed in the roots of overexpressor lines and in overexpressor plants upon
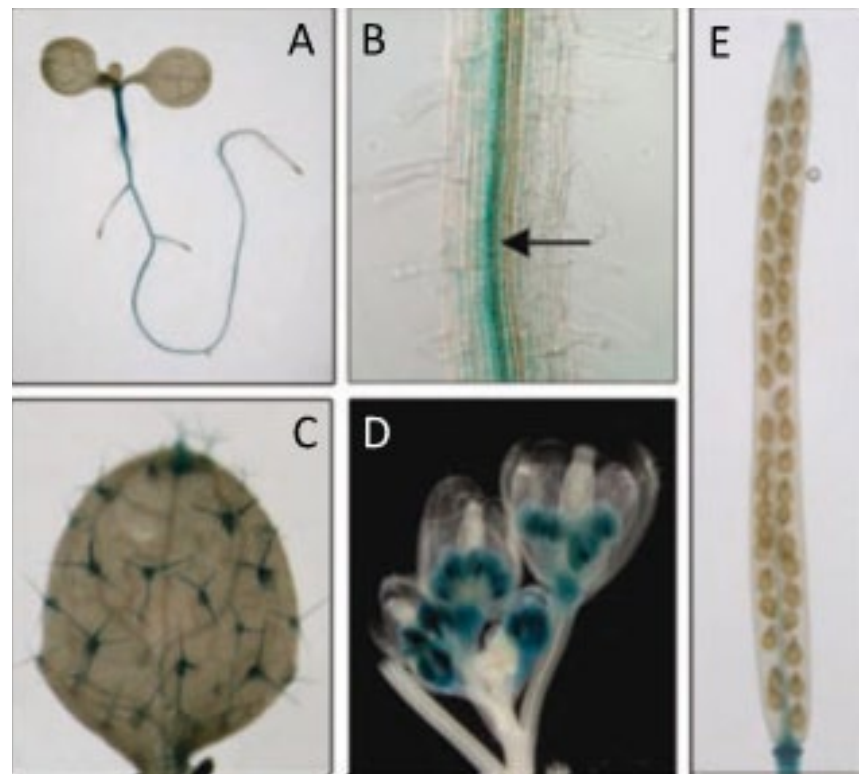

Figure 28. CYP82C2 promoter-driven GUS expression in $A$. thaliana.

(A) in 6-day-old seedlings; (B) in root vascular tissues of 6-day-old seedlings; (C) in leaf of 14-day-old seedlings; (D) in anthers of open flowers; (E) in silique. Reprinted from Liu et al. (2010) with permission from Nature Publishing Group.

MeJA treatment. Indole acetic acid and camalexin synthesis were not affected by the expression of CYP82C2.

CYP82G1, the single representative of this subfamily, was shown to cleave the diterpenoid $(E, E)$-geranyllinalool and the sesquiterpenoid $(E)$-nerolidol into the acyclic volatile $C_{16}$-homoterpene 4,8,12-trimethyltrideca-1,3,7,11-tetraene (TMTT) and the $\mathrm{C}_{11}$-homoterpene 4,8-dimethyl-1,3,7-nonatriene (DMNT), respectively (Lee et al., 2010) (Figure 29). TMTT and DMNT were released in response to herbivory or produced constitutively in Arabidopsis and were shown to be involved in the so-called tritrophic interaction between plants, herbivores, and their parasitic predators (Van Poecke et al., 2001; Kappers et al., 2005). Lee and co-workers (2010) identified a number of candidate P450s for the oxidative cleavage through an in silico search for genes coexpressed with geranyllinalool synthase (GES). A T-DNA insertion null mutant allele in one of the candidate genes, CYP82G1, showed accumulation of geranyllinalool but not TMTT. Furthermore, constitutive expression of full-length CYP82G1 in the null mutant background restored TMTT formation. Transgenic lines expressing the reporter $\beta$-glucuronidase (GUS) under control of the CYP82G1 promoter showed reporter activity after insect feeding, wounding or inoculation with pathogenic bacteria. Heterologous expression of CYP82G1 in the WAT11 yeast strain which carries the chromosomally integrated Arabidopsis ATR1, resulted in TMTT and DMNT production with $(E, E)$-geranyllinalool and $(E)$ nerolidol as substrates, albeit at relatively low catalytic efficiency. Other acyclic terpenoid substrates with the double bonds or the hydroxyl function in different positions were tested, but showed either poor conversion or no product formation. In the absence of $\mathrm{C}_{4}$-cleavage products (but-1-en-3-one) or intermediates of the 

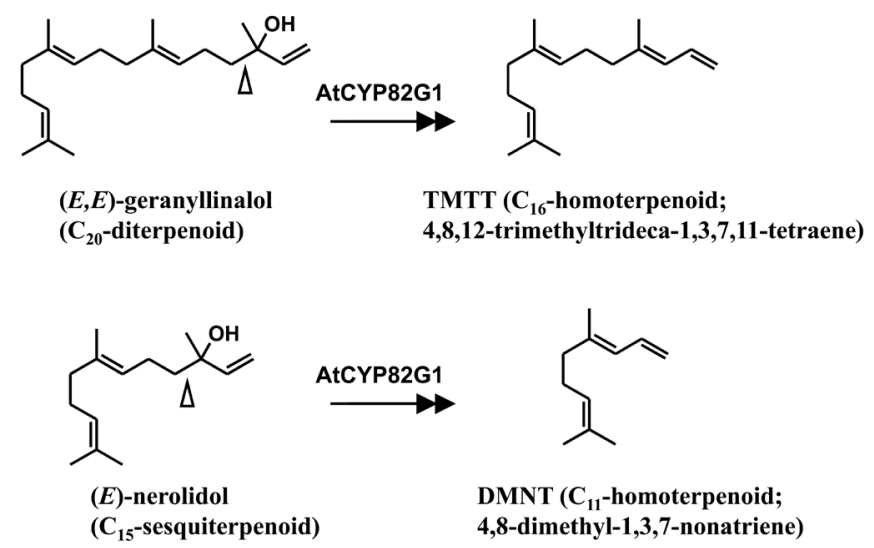

Figure 29. C-C cleavage reactions for the formation of homoterpenoids from acyclic diterpenoid and sesquiterpenoid substrates by Arabidopsis CYP82G1

degradation reaction, structural modeling and docking of both substrates was used to further corroborate a reaction mechanism suggested to follow oxidative-bond cleavage with syn-elimination ( $\beta$-elimination) (Boland et al., 1998). Cleavage of C-C is an unusual reaction catalysed by $\mathrm{P} 450$ enzymes. Further examples which may follow a mechanistically similar route to the reaction catalysed by CYP82G1 are found in the same CYP71 clan with CYP71AJ1 converting the furanocoumarin (+)-marmesin to psoralen by oxidative cleavage in Ammi majus (Larbat et al., 2007) and CYP72A1 from Catharanthus roseus, in oxidative conversion of the alkaloid loganin to secologanin (Irmler et al., 2000).

\section{CYP83}

The CYP83 family appears late in plant evolution and is only found in some dicots. CYP83 succeeds CYP79 in glucosinolate biosynthesis. There are two CYP83s in the Arabidopsis genome, CYP83A1 and CYP83B1. They share $65 \%$ amino acid sequence identity, and group between the CYP71A and CYP71B subfamilies in phylogenetic trees. Despite the fact that they phylogenetically belong to the CYP71 family, they have retained their initial naming to avoid conflicts in the literature. In the presence of thiol compounds, CYP83B1 catalyzes the initial conversion of indole3-acetaldoxime to the corresponding S-alkyl-thiohydroximate in indole glucosinolate biosynthesis (Bak et al., 2001, Bak and Feyereisen, 2001) (Figure 25). In the absence of thiol compounds, the product of the enzymatic reaction inactivates the enzyme. Indole-3-acetaldoxime is an intermediate in indole-3-acetaldoximedependent IAA biosynthesis (reviewed by Normanly and Bartel, 1999; Sugawara et al., 2009). In agreement with this, the CYP83B1 knock-out mutant, rnt1-1, (Figure 30 ) is characterized by a high IAA phenotype (apical dominance) and low indole glucosinolate levels. Conversely, ectopic over-expression of CYP83B1 induces a low IAA phenotype (reduced apical dominance) and elevated indole glucosinolate levels (Bak et al., 2001, Bak and Feyereisen, 2001). rnt1-1 is allelic to the well-characterized auxin mutant sur2, known to hyperaccumulate free IAA (Barlier et al., 2000; De-
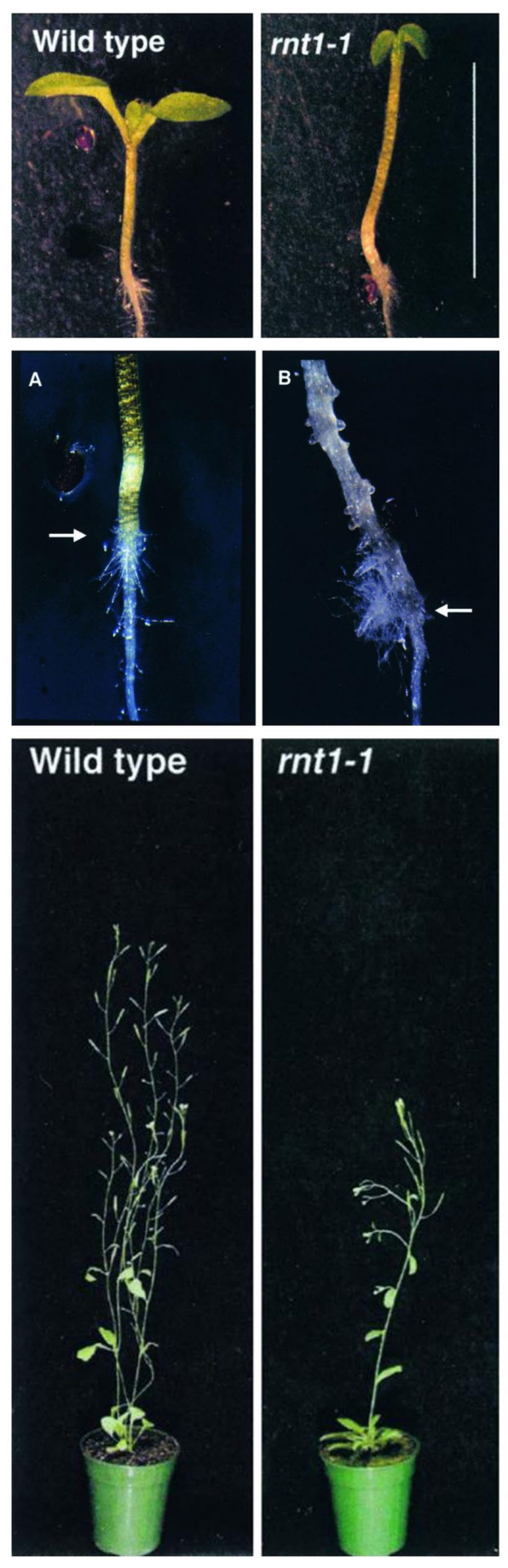

Figure 30. The rnt1-1 phenotype induced by knock-out of CYP83B1. Reprinted from Bak and Feyereisen (2001).

(A) 1 week old $r n t 1-1$ seedlings have increased hypocotyl length and epinastic cotyledons.

(B) After two weeks, exfoliation of the hypocotyl begins at the root-hypocotyl junction $(\neg)$. Secondary roots initiate from the hypocotyl, and there is an enhanced formation of secondary roots and root hairs.

(C) 6 weeks old rnt1-1 plants have increased apical dominance due to elevated IAA levels. Typically, the plants have a reduced height, an increased number of epinastic rosette leaves and a single inflorescence. 
larue et al., 1998). The CYP83B1 mutants and over-expression analysis thus concur to demonstrate that CYP83B1 occupies the metabolic branch-point between IAA and indole glucosinolate biosynthesis (Bak et al., 2001, Bak and Feyereisen, 2001). Ectopic expression of the CYP83A1 cDNA in rnt1-1 rescues the high IAA phenotype, and restores indole glucosinolate levels back to wildtype levels, which suggests that CYP83A1 is functionally redundant to CYP83B1 (Bak et al., 2001). However, CYP83A1 has a 50 fold higher $K_{m}$ for indole-3-acetaldoxime than CYP83B1. Thus under normal conditions CYP83A1 is not functionally redundant to CYP83B1 (Bak et al., 2001, Nauer et al., 2003). In addition to the tryptophan-derived indole-3-acetaldoxime, CYP83B1 also metabolizes the tyrosine-derived $p$-hydroxyphenylacetaldoxime and the phenylalanine-derived phenylacetaldoxime to the corresponding $S$-alkyl-thiohydroximates, but with lower affinity than for indole-3-acetaldoxime (Bak et al., 2001; Hansen et al., 2001). Conversely, $p$-hydroxyphenylacetaldoxime is a preferred substrate for CYP83A1 as compared to CYP83B1. Over-expression data and knock-out mutants show that aliphatic glucosinolates are derived from CYP83A1 and not CYP83B1 (Naur et al., 2003, Hemm et al., 2003). Thus CYP83A1 and CYP83B1 have overlapping but not identical substrate specificities, where, in plants, CYP83B1 is involved in indole glucosinolate biosynthesis and CYP83A1 in aliphatic glucosinolate biosynthesis (Bak et al., 2001; Nauer et al., 2003).

The sur2 mutant was originally identified in a forward genetic screen based on a high auxin phenotype, and subsequently as rnt1-1 in a systematic reverse genetic screen for P450 mutants (Winkler et al., 1998). Additional forward genetic screens, which were not focused on auxin selection criteria, resulted in the identification of CYP83B1 as ATR4 (Altered Tryptophan Regulation4) based on altered feedback regulation of tryptophan biosynthesis in the presence of the toxic tryptophan analog 5-methyl-tryptophan (Smolen and Bender, 2002) and as RED1 (Red Light Elongated1) based on a screen for perturbed phytochrome $B$ signaling (Hoecker et al., 2004). The many alleles for CYP83B1, rnt1-1, sur2, ATR4 and RED1, indicated that CYP83B1 is involved in a complex metabolic grid and regulatory network. A systems biology approach combining transcriptomics, metabolomics and biochemistry revealed pronounced effects on several metabolic grids including the intersection between secondary metabolism, cell wall turnover, hormone metabolism and stress responses (Morant et al., 2010). The observed metabolic and transcriptional crosstalk with CYP83B1 acting as a hub indicates that it is regulated by a complex interaction of both positively and negatively acting transcription factors. Thus, the phenotype of CYP83B1 mutants cannot be explained merely by elevated levels of auxin, but also that of altered ethylene and abscisic acid levels, that leads to drought responses in the absence of a water deficiency (Morant et al., 2010).

CYP83A1 is not involved in the production of indole-3-acetaldoxime and thus not in the complex IAA network. CYP83A1 mutants and CYP83A1 overexpressors do not display a high auxin phenotype. In a screen based on altered fluorescence under UV light, knock-out lines of CYP83A1 were identified and designated ref2. These plants have no visual phenotype in comparison to wild type, but were shown to have reduced levels of sinapoyl malate and the absence of aliphatic glucosinolates in the leaves, indicating a link between aliphatic glucosinolates and phenylpropanoids in A. thaliana (Hemm et al., 2003).

\section{CYP84}

The CYP84 family appears in Magnoliids. It is represented by two members in Arabidopsis, CYP84A1 and CYP84A4. CYP84A1 was the first CYP gene in the phenylpropanoid pathway to be characterized in Arabidopsis. A forward genetic approach based on the screening of EMS mutants for a defect in sinapic esters accumulation led to the isolation of mutants at a locus designated SIN1 (Chapple et al., 1992; Fraser and Chapple, 2011). The sin1 mutant phenotype included an altered lignin composition with absence of synringyl units, lack of blue fluorescence due to sinapoyl malate in the leaf epidermis, and lack of sinapoyl choline in seeds (Figure 31). A biochemical analysis of the mutants, and complementation assays, revealed that the block in sinapoyl malate biosynthesis affected the 5-hydroxylation step of the phenolic ring. Based on these data, the SIN1 locus was then re-named FAH1 (for ferulic acid hydroxylase).

Additional fah1 alleles were isolated from the same EMS mutant collection (Ruegger and Chapple, 2001), and also from a T-DNA tagged library on the basis of red fluorescence of the mutants under UV-light (Meyer et al., 1996). The T-DNA interrupted sequence was isolated and shown to encode CYP84A1. A genomic CYP84A1 sequence complemented the fah1 mutation. Expression of CYP84A1 is significantly delayed compared to CYP73A5 (encoding cinnamate hydroxylase). It is only expressed in the oldest rachis internodes, during maturation of sclerified parenchyma, and results in an increased syringyl lignin content (Meyer et al., 1998). Over-expression of CYP84A1 in the mutant fah1 plants devoid of syringyl lignin, suppresses the tissue-specific expression of the $F A H$ gene, and leads to plants with increased syringyl lignin compared to wild type. Thus, the temporal and tis-
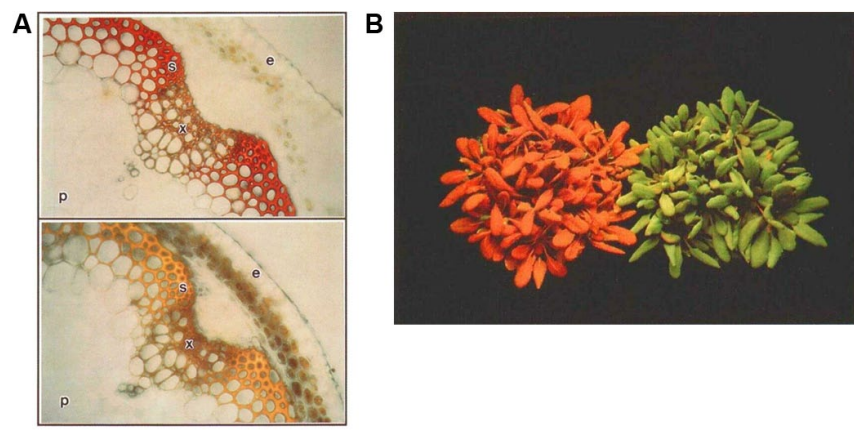

Figure 31. The $\sin 1$ (fah1) mutant phenotype. Reprinted from Chapple et al. (1992).

Impact of the mutation on the staining of lignin with the Mäule reagent which produces a red coloration in the presence of syringyl units. Upper panel: wild type. Lower panel: sin1 mutant. The position of the pith (p), sclerified parenchyma (s), xylem (x), and epidermis (e) are indicated.

Wild-type and $\sin 1$ mutant photographed under UV light. Wild-type (right) and $\sin 1$ mutant (left) plants were photographed using a $365 \mathrm{~nm}$ transilluminator as a light source and a pale yellow barrier to remove reflected UV light. The green color of the wild-type plants is due to the fluorescence of sinapoyl malate in the leaves' upper epidermis and appears green rather than blue because of the filter employed during photography. The red color of the mutant is due to the UV-induced chlorophyll fluorescence that is revealed in the absence of sinapoyl malate. 
sue-specific expression of the CYP84A1 gene seems to control the composition of lignin polymers in Arabidopsis. Interestingly, over-expression studies carried out in fah1 and in different plant species showed that the CaMV35S promoter fails to drive high levels of CYP84A1 expression in tissues undergoing lignification. Much higher levels of expression were attained using the tissuespecific promoter of CYP73A5 that leads to the formation of lignin derived mainly from syringyl units (Meyer et al., 1998; Marita et al., 1999; Franke et al., 2000). CYP84A1 driven by the CYP73A5 promoter thus appeared as target of choice for manipulation of lignin composition (Huntley et al., 2003; Stewart et al., 2009). The full impact of CYP84A1 suppression and over-expression on lignification of different cellular types and on vascular development in Arabidopsis was evaluated and discussed in detail by Patten et al. (2010).

Other experiments with the fah1 mutants demonstrated that, in Arabidopsis, sinapoylmalate is an important UV-B screen, more powerful than flavonoids, (Landry et al., 1995). Complementation of the mutation with different constructs, including the genomic sequence and different alternative promoters, indicated that CYP84A1 expression in leaves and de novo synthesis of sinapo$\mathrm{yl}$ choline are dependent on a regulatory domain that is located 3 ' of the stop codon (Ruegger et al., 1999). This region is not required for expression of CYP84A1 in the embryo and accumulation of sinapoyl choline in the seeds. The same study showed that CYP84A1 is regulated distinctly from other phenylpropanoid genes, and strongly dependent on light in young seedlings. CYP84A1 expression, however, is not rate limiting for the biosynthesis of sinapate esters in seedlings and developing siliques.

The impact of the fah1 mutant on resistance to the bacterial pathogen Pseudomonas syringae defense has been evaluated by Hagemeier et al. (2001). Sinapoyl malate was found to strongly decrease upon $P$. syringae infection in the wild type, inversely correlated with tryptophan-derived products. In the mutant, besides the lack of sinapoyl malate, no major modification in soluble or wall-bound metabolites was observed in infected plants. Accordingly, the fah1 mutant showed no alteration in its disease resistance phenotype. A broader scale investigation, based on transcriptome analysis and metabolic profiling of a T-DNA insertion mutant, however, leads to very different conclusions. It reports pleiotropic effects of the mutation (Huang et al., 2009). These include deep modification in the expression of structural, metabolic and regulatory genes in the mutant, in particular related to flowering and seed development, a stunted inflorescence phenotype of the mutant under drought and $\mathrm{NaCl}$ stress conditions, and increased susceptibility to fungal pathogens potentially related to a decrease in salicylic acid conjugates and glucosinolates.

The catalytic function of CYP84A1 was originally assumed from the mutant analysis, but not confirmed with recombinant enzyme. When, several years later, the protein was successfully expressed in yeast, it turned out that its catalytic function was different from initially expected, leading to a significant revision of the lignin biosynthesis pathway. Ferulic acid appeared as a very poor substrate of recombinant CYP84A1, while coniferaldehyde and coniferyl alcohol were hydroxylated with a much higher efficiency (Humphreys et al., 1999). The efficiency of the metabolism of coniferaldehyde or coniferyl alcohol was about 1000 times that of ferulic acid. This suggested that the latter was probably not the substrate in vivo. This updated catalytic function of CYP84A1
(Figure 32) provided an explanation to many results considered as enigmatic on the basis of the previous model.

Optimized expression of CYP84A1 in yeast (Jian and Morgan, 2004) and active site architecture based on homology modeling (Rupasinghe et al., 2003) have been described. The fah1 mutants were also shown to be very useful tools to probe the function of genes from lycophytes by complementation approaches (Weng et al., 2008).

CYP84A4 is the second member of the CYP84 family. CYP84A4 has only $64 \%$ amino acid identity with CYP84A1 and its gene has one intron less. CYP84A4 is expressed in hypocotyl, stem, petiole, roots and seeds, which suggests a role in vascularization, but there is so far no published information concerning its specific function.

\section{CYP85}

CYP85A was first identified in tomato and shown to catalyse C-6 oxidation in BR biosynthesis (Bishop et al., 1996; Bishop et al., 1999). Brassinosteroids (BRs) are poly-hydroxylated steroid hormones that promote plant growth and cell division. The biosynthesis of BRs from campesterol requires multiple hydroxylation/oxidation reactions catalysed by P450s and a simplified biosynthesis pathway is shown in Figure 33. Mutations in P450s that catalyse BR synthesis normally result in dwarfed plants that can be rescued to a wild-type phenotype by exogenous application of BRs. In Arabidopsis two CYP85A genes are present and are somewhat functionally redundant with the null cyp85a1 T-DNA tagged mutant having no easily observable phenotype and cyp85a2, another T-DNA tagged mutant, exhibiting a weakly dwarfed phenotype. As shown in Figure 34, cyp85a1/cyp85a2 double mutants are, however, severely dwarfed (Nomura et al., 2005; Kim et al., 2005; Kwon et al., 2005). Both CYP85A1 and CYP85A2 can convert 6-deoxocastasterone to castasterone, but only CYP85A2 can synthesize the most bioactive brassinosteroid, brassinolide (Shimada et al., 2001; Nomura et al., 2005; Kim et al., 2005b; Kwon et al., 2005). The double mutant has been useful in defining which BRs are bioactive and indicates that both castasterone and brassinolide are bioactive BRs, with brassinolide having more biological activity (Nomura et al., 2005).

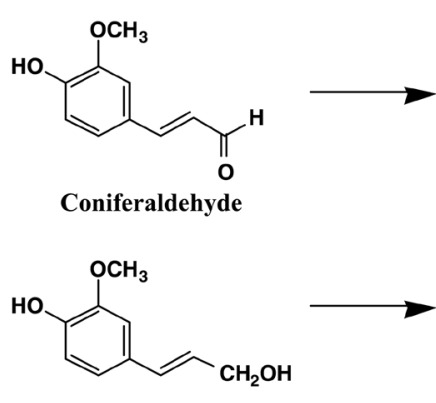

Coniferyl alcohol

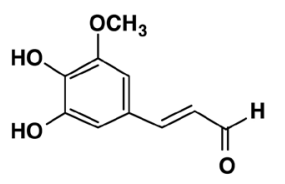

5-Hydroxyconiferaldehyde<smiles>COc1cc(/C=C/CO)cc(O)c1O</smiles>

5-Hydroxyconiferyl alcohol
Figure 32. The reactions catalyzed by CYP84A1. 

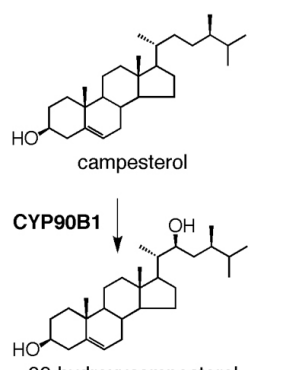

Other P450s assoicated with;

BR synthesis

CYP90A1, CYP724A1

BR metabolism
CYP72C 1

22-hydroxycampesterol

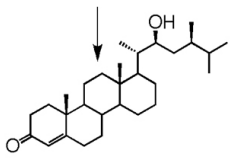

(22S,24R)-22-hydroxyergost-4-en-3-one

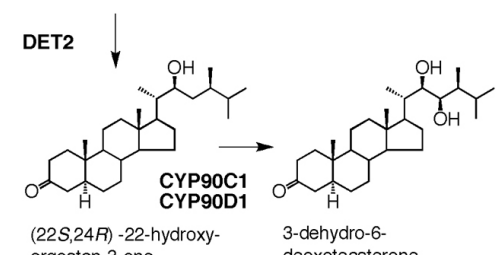

ergostan-3-one deoxoteas
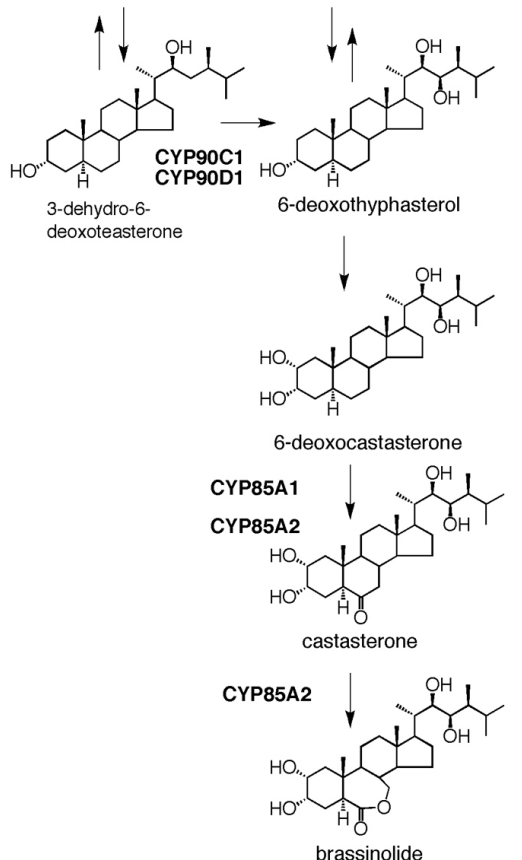

(most bioactive brassinosteroid)

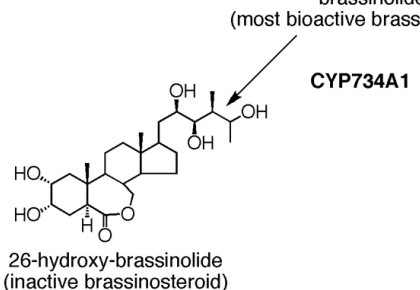

(inactive brassinosteroid)

Figure 33. Simplified route of brassinosteroid biosynthesis and metabolism

Location of CYPs on BR synthesis and metabolism pathway, major intermediates are shown. Location of CYP90A1, CYP724A1 and CYP72C1 need to be clarified.
Expression analysis has shown that these P450s are expressed in tissues undergoing elongation and division, and thus correlates well with the dwarf phenotype (Shimada et al., 2003; Castle et al., 2005). As discussed later (in CYP90 section), CYP85A genes along with the other BR biosynthesis genes are coordinately regulated at the transcriptional level via a negative feedback mechanism (Bancos et al., 2002).

\section{CYP86}

In Arabidopsis the typical non-A P450 family CYP86 is represented by five CYP86As, two CYP86Bs and four CYP86Cs. Two genes initially classified as CYP86Ds have been renamed CYP94D. The CYP86B2 gene that was missed in the initial annotation due to its tandem location with CYP86B1 is now recorded. The first characterized member of the CYP86 family, CYP86A1, was isolated based on its protein sequence homology with fatty acid-metabolizing CYP4s and CYP52s from mammals and fungi, respectively (Benveniste et al., 1998). Consistent with this sequence homology, CYP86A1 has been shown to catalyze the $\omega$-hydroxylation of $\mathrm{C} 12$ to $\mathrm{C} 18$ fatty acids in recombinant yeast. A similar activity was shown for CYP86A8 (Wellesen et al., 2001). When heterologously expressed in Sf9 insect cells using baculovirus-mediated expression systems, all five members of the CYP86A subfamily where able to catalyze the $\omega$-hydroxylation of lauric acid, to metabolize oleic acid and to bind fatty acids of different chain length and saturation, including the oxygenated 9,10-epoxystearic and 9,10-dihydroepoxystearic acids (Duan and Schuler, 2005; Rupasinghe et al., 2007). Thus the sequence conservation of the CYP86A subfamily compared to the fatty acidmetabolizing CYP4 and CYP52 families also reflects some conservation of enzyme function. A detailed analysis of the evolution and the promoter sequences from the CYP86A subfamily was published by Duan and Schuler (2005).

A decade after the description of CYP86A1 as $\omega$-hydroxylase, the in vivo function as hydroxylase of root suberized tissue (horst) was shown in Arabidopsis T-DNA insertion mutants (Höfer et al., 2008). The horst mutants had, compared to the wild type, a significant reduction in $\omega$-hydroxy fatty acids and $\alpha, \omega$-dicarboxylic fatty acids with a chain length from C16 to C20 in aliphatic root suberin. Since the $\omega$-hydroxy fatty acids are precursors for the biosynthesis of $\alpha, \omega$-dicarboxylic fatty acids, the reduction in $\alpha, \omega$ dicarboxylic fatty acids is an expected secondary effect. The total amount of aliphatic root suberin in the horst mutants was reduced by about $60 \%$. Consistent with its involvement in aliphatic root suberin biosynthesis, expression of CYP86A1 was only detected in the root (Duan and Schuler 2005; Höfer et al., 2008). In wild-type Arabidopsis transformed with a Prom $_{\mathrm{CYP86A1}}$ : GUS construct, GUS activity could be located to the endodermis of the primary root (Höfer et al., 2008). At the subcellular level, CYP86A1:GFP fusion protein was shown to be targeted to the endoplasmic reticulum in Nicotiana benthamiana epidermal cells. Further proof of function of CYP86A1 was provided by an ectopic over-expression in Arabidopsis (Li et al., 2007). When co-expressed with GPAT5, an acyltransferase associated with suberin biosynthesis, the stem cutin of transgenic Arabidopsis lines showed an increased amount of C16 and C18 $\alpha, \omega$-dicarboxylic fatty acids and new C20 and C22 $\alpha, \omega$-dicarboxylic fatty acids which are not present 

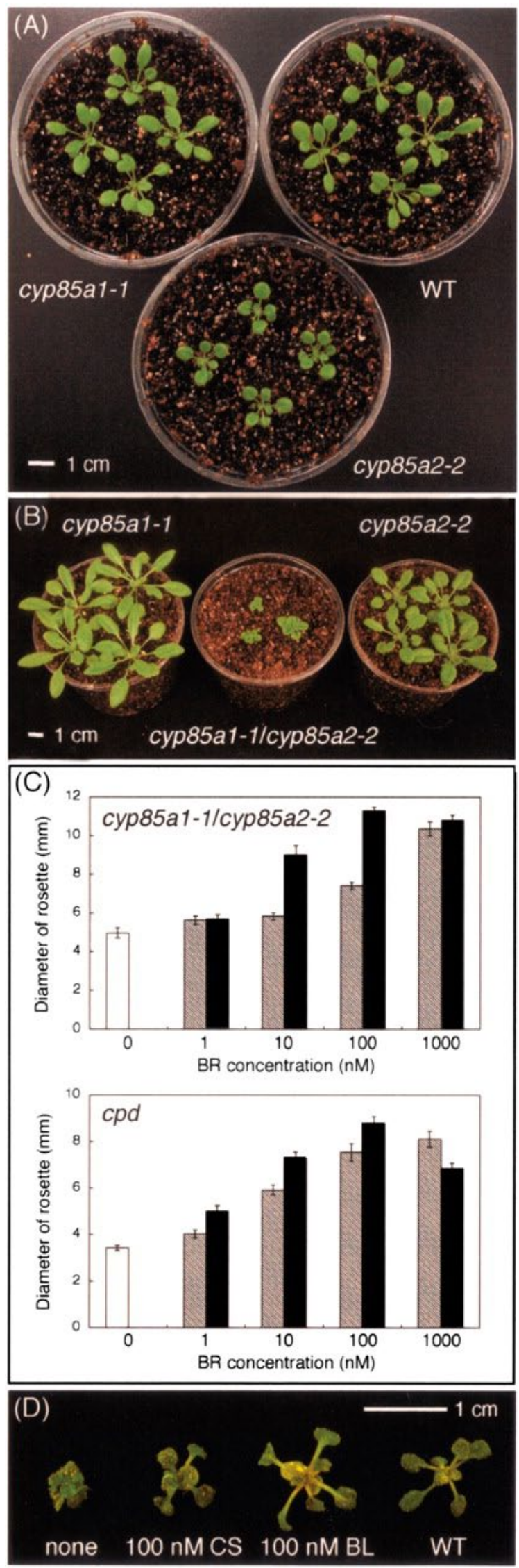

in the wild type. As a result of this altered cuticle composition, TEM analysis showed a replacement of the normally compact and electron-dense cuticle by a diffuse 2-3 times thicker multilayered structure. It is worth mentioning that CYP86A33, a Solanum tuberosum homolog of CYP86A1, was also shown to be involved in suberin biosynthesis (Serra et al., 2009). Interestingly, the altered suberin composition of the potato tuber periderm from plants where CYP86A33 was down-regulated by RNAi leads also to an altered suberin ultrastructure and, in addition, to a higher water permeability of the isolated tuber periderm.

Another gene associated with suberin biosynthesis in Arabidopsis is CYP86B1. The cyp86b1 T-DNA insertion and RNA interference-silenced lines have a strong reduction in aliphatic $\omega$-hydroxy fatty acids and $\alpha, \omega$-dicarboxylic fatty acids with a chain length of C22 and C24 in root suberin and seed coat when compared to the wild type (Compagnon et al., 2009). Therefore, these lines were named root aliphatic plant hydroxylase (ralph). Despite the altered seed coat polyester and root suberin composition, neither the seed coat permeability, nor the ion content

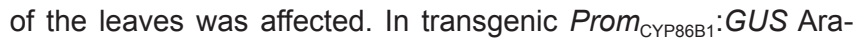
bidopsis lines, GUS activity was observed in the endodermis of the primary root, with a pattern similar to $\operatorname{Prom}_{\mathrm{CYP86A}}$ : GUS lines. Furthermore a GUS signal was detected in stomatal guard cells of pedicels, in the hilum of mature seeds and in specific tissues of the silique and flower. A CYP86B1:YFP fusion protein was shown to be targeted to the endoplasmic reticulum of pavement cells and the chloroplasts of guard cells of $N$. benthamiana leaves. In an in vitro import assay, CYP86B1 was found incorporated in the outer envelope of the chloroplast (Watson et al., 2001). As shown for CYP86A1, an ectopic overexpresssion of CYP86B1 together with GPAT5 leads to an altered stem cutin in the transgenic lines with an incorporation of novel compounds that are not present in

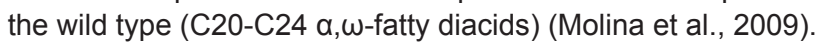

Like suberin, cutin is an essential plant biopolyester derived from fatty acids and fatty acid derivatives. Three members of the CYP86A subfamily, CYP86A2, CYP86A4 and CYP86A8, were shown to be involved in cutin biosynthesis. A T-DNA insertion in CYP86A4 was shown to result in a $45-58 \%$ reduction of 16 -hydroxypalmitate, 10,16-dihydroxypalmitate, and 1,16-hexadecanedioic acid in flower cutin (Li-Beisson et al., 2009). For CYP86A4 and CYP86A7, it was shown that both genes are rapidly induced by the transcription factor WIN1 (WAX INDUCER1) (Kannangara et al., 2007). The highest expression of CYP86A4 and CYP86A7

Figure 34. Knock-out phenotypes of Arabidopsis CYP85A1 and CYP85A2.

(A) 24-day-old seedlings of cyp85a1, cyp85a2 and WT.

(B) 31-day-old single and double mutant of cyp85a1 and cyp85a2.

(C) The effects of BL and CS on the cyp85a1/cyp85a2 double mutant and the cpd mutant. Ten-day-old seedlings were treated on a medium containing either CS (striped bar) or BL (black bar). After 4 days of treatment, the diameters of the rosettes were measured.

(D) The cyp85a1-2/ cyp85a2-2 mutant treated on a medium containing $100 \mathrm{nM}$ CS or $100 \mathrm{nM} \mathrm{BL}$ for 4 days and WT seedlings grown on the medium without BRs. Reprinted from Nomura et al. (2005) with permission from the American Society for Biochemistry and Molecular Biology. 
in response to WIN1 was detected in all parts of the flower and in the silique. Over-expression of WIN1 in Arabidopsis led to a higher total wax and cutin content in flowers and leaves; silencing of WIN1 had the opposite effect. It was thus assumed that CYP86A7 might also be associated with cutin biosynthesis in the inflorescence.

A striking phenotype, including fusions between rosette leaves and in inflorescences, drew attention to a transposon-tagged Arabidopsis mutant of CYP86A8 (Wellesen et al., 2001) (Figure 35). As mentioned above, in recombinant yeast, CYP86A8 catalyzes the $\omega$-hydroxylation of $\mathrm{C} 12$ to $\mathrm{C} 18$ fatty acids. It thus seems likely to contribute to the biosynthesis of cutin in the epidermis for preventing postgenital organ fusion. The so-called lacerata (Icr) mutation is pleiotropic. Other characteristics of the Icr plants include abnormalities in cell morphology, retarded formation of trichomes, delayed senescence, lower height and increased number of shoots which seems indicative of reduced apical dominance. The molecular basis of these defects, which might be related to the absence of some lipid-derived signal, is however not understood. As with other fusion mutants, the Icr plants support pollen germination on the leaf surface.

CYP86A2 mutants initially came into the spotlight because of an altered plant defense phenotype, but also turned out to have altered cutin biosynthesis. Arabidopsis insertion lines are characterized by an extremely up-regulated expression of bacterial type III genes and enhanced disease symptoms after inoculation with Pseudomonas syringae (Xiao et al., 2004). Therefore the mutants were called aberrant induction of type three genes (att1). The att1 mutant shows a general reduction of all cutin monomers, resulting in a total cutin amount of just $30 \%$ compared to the wild type. The cuticle membrane has a loose ultrastructure with increased permeability to water vapor, and a complete permeability to toluidine-blue (Galbiati et al., 2008) (Figure 36). Analysis of the seed coat polyester of att1 revealed a reduction in C16:0, C18:1 and C18:2 $\alpha, \omega$-dicarboxylic fatty acids, but not in the corresponding $\omega$-hydroxy fatty acids (Molina et al., 2008). Interestingly the seed coat permeability to tetrazodium salts was not affected by the altered seed coat polyester composition. So far, the results from the cutin and seed coat polyester analyses do not really explain the impact of ATT1 on disease resistance. They suggest that one role of CYP86A2 is the synthesis of cutin-related fatty acids that may repress bacterial type III gene expression (Xiao et al., 2004). During plant development, the ATT1 promoter is active in still developing seeds, in the inner integument layer facing the endosperm (Molina et al., 2008). It co-localizes with the cuticle observed by TEM analysis (Beeckman et al., 2000). In a gene-trap line, expressing the GUS gene instead of CYP86A2, GUS activity was also found in the developing seeds (Galbiati et al., 2008). Furthermore, GUS activity was found throughout seedling development in all organs. In immature leaves an intense stomatal guard cell-specific signal was observed, decreasing in fully expanded leaves. Also guard cell-specific GUS expression was revealed in unfertilized and pollinated flower organs and carpels of developing siliques. The stigmatic tissue- and ovule-specific GUS expression found in flowers was confirmed by microarray-based expression profiling of Arabidopsis pistils (Tung et al., 2005). In addition, an intense staining of the abscission zone and styles of the developing siliques was observed (Galbiati et al., 2008).
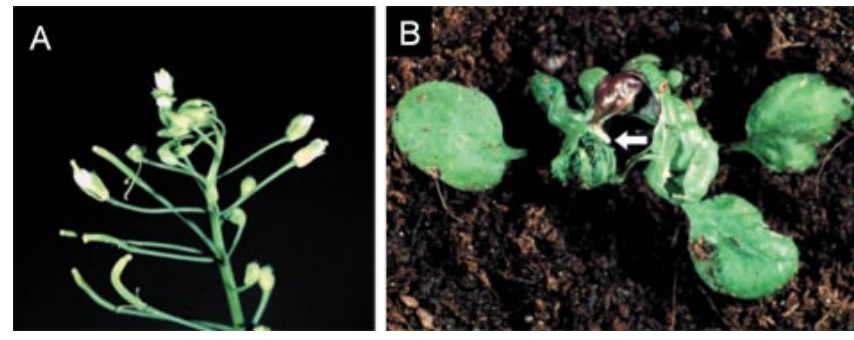

Figure 35. Features of the phenotype of /cr mutants.

(A) Inflorescence of an Icr plant exhibiting organ fusion. The Icr mutant is generally fertile, although partially filled siliques are sometimes observed.

(B) An example of the strong leaf fusion that caused tearing of tissues that were not directly implicated in the fusion during growth of the $/ \mathrm{cr}$ plant. The arrow shows the broken petiole; note that the detached leaf blade did not senesce. Reprinted from Wellesen et al. (2001) with permission from the National Academy of Sciences.
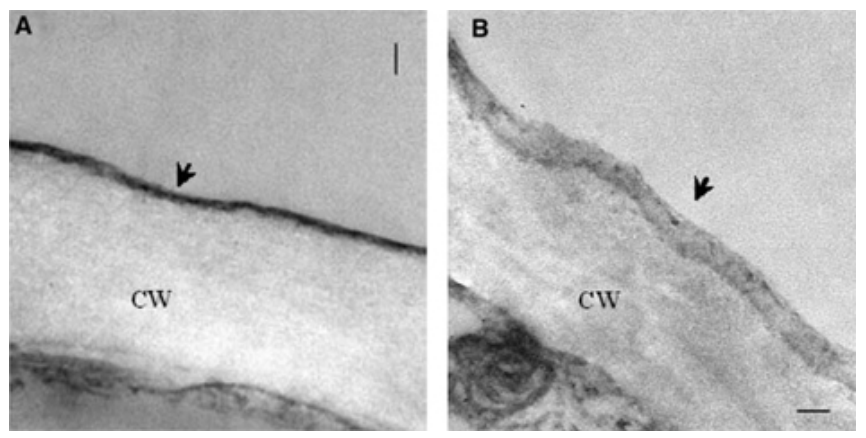

Figure 36. The null cyp86a2-1 mutant displays altered cuticle membrane ultrastructure.

Ultrastructure of the wild-type Col-gl (A) and the cyp86a2-1 mutant (B) cuticle membrane from an epidermal cell. The cuticle (arrowhead) and cell wall (CW) are shown. Note the lower electron density of the cyp86a2-1 cuticle membrane, which was found in all samples examined, compared with the dense, compact cuticle in the wild-type plant. Reprinted from Xia et al. (2004) with permission from the Nature Publishing Group.

Recently, a member of the CYP86C subfamily, CYP86C3, was shown to catalyze the $\omega$-hydroxylation of $\mathrm{C} 12$ to $\mathrm{C} 16$ fatty acids when expressed in insect cells (Kai et al., 2009). No further studies have yet been reported for this subfamily.

\section{CYP87}

The function of the single CYP87 gene in Arabidopsis is currently unknown. CYP87 belongs to the CYP85 clan that includes many P450s involved in hormone biosynthesis. It seems to have evolved with the emergence of Angiosperms (Nelson and Werck-Reichhart, 2011). Absence of duplication in this family is indicative of an essential function. A single reference to a study on CYP87 in rice coleoptiles indicates a role in auxin signaling in the regulation of coleoptile growth (Chaban et al., 2003), which fits with the general assumption that most members of the CYP85 clan are involved in hormone metabolism. 


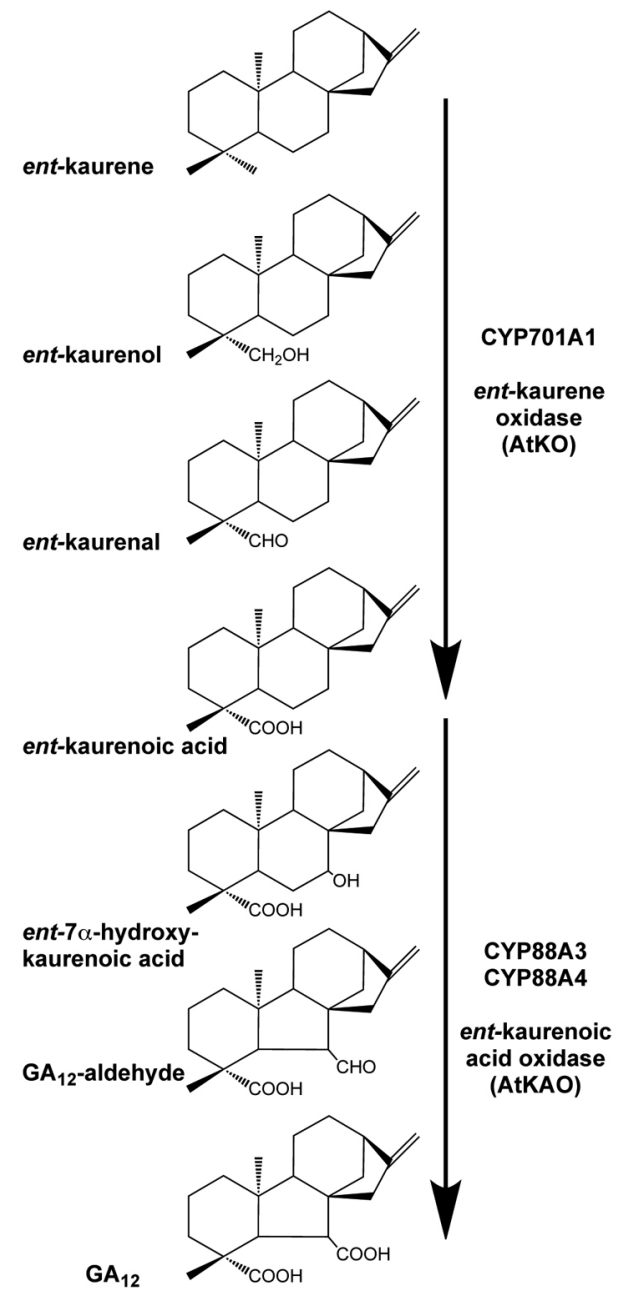

Figure 37. Conversions catalyzed by CYP88 and CYP701 P450s in gibberellin biosynthesis.

CYP701A1 the ent-kaurene oxidase (AtKO), catalyzes three successive oxidations at C-19. This is followed by CYP88A3/CYP88A4 ent-kaurenoic acid oxidase (AtKAO) that carryout three successive oxidations on C-7.

\section{CYP88}

Arabidopsis has two CYP88 genes that are involved in the synthesis of gibberellins (GAs), which are tetracyclic diterpenes. GAs promote shoot elongation, parthenocarpy, flowering and germination. Plants defective in GA synthesis can be non-germinating and dwarfed. CYP88A3 and CYP88A4 encode ent-kaurenoic acid oxidases (KAOs) that are involved in GA biosynthesis converting ent-kaurene to GA12 (see Figure 37) (Helliwell et al., 2001a).

CYP88As belong to the CYP85 clan and are evolutionally related to P450s involved in brassinosteroid biosynthesis. CYP88s are found in liverwort and lycopods (Nelson and Werck-Reichhart, 2011) but not in P. patens (where CYP701 is present), which indicates that CYP88 emerged with the divergence of vascular plants from the bryophyte lineage (Hirano et al., 2007). While both CYP88A3 and CYP88A4 were shown to catalyze the conversion of ent-kaurenoic acid to $\mathrm{GA}_{12}$ when expressed in yeast, (Helliwell et al., 2001b), their low sequence identity of $\sim 76 \%$ suggests a possible differential substrate preference for different GAs. Dwarf mutants associated with CYP88A genes in Arabidopsis have not been described, which is most likely due to gene redundancy. Such redundancy can be explained as CYP88A3 and CYP88A4 are both expressed in all aerial parts of the plant with higher expression in inflorescence stems, inflorescences and siliques. In other species, mutations in CYP88 genes lead to typical GA-related dwarfism phenotypes, e.g. in barley ( $g r d 5)$ and maize (dwarf3) (Helliwell et al., 2001a; Winkler and Helentjaris, 1995).

\section{CYP89}

The function of the seven CYP89A genes, four of which form a small cluster on chromosome I of Arabidopsis, has not been investigated. CYP89s belong to the CYP71 clan. They appeared early with the evolution of Angiosperms and are detected in Magnoliids (Nelson and Werck-Reichhart, 2011). They seem to derive from CYP77s, which are fatty acid in-chain hydroxylases.

\section{CYP90}

CYP90s belong to the CYP85 clan. There are four CYP90 subfamilies in Arabidopsis all involved in BR biosynthesis and each of them having a single member (Figure 33).

The T-DNA-tagged constitutive photomorphogenic and dwarfism (cpd) mutant is defective in CYP90A1 and was a key mutant in helping to define the role of BRs in plant development (Szekeres et al., 1996). cpd plants are severely dwarfed and exhibit male sterility. Dark-grown cpd plants have a short hypocotyl, no apical hook, open cotyledons, these phenotypes suggested a defect in photomorphogenesis and de-etiolation. The $c p d$ phenotype can be rescued to wild type with exogenous application of BRs, with initial experiments suggesting that this mutant was defective in C-23 hydroxylation (Szekeres et al., 1996). As discussed later, however, more recent analysis has shown that CYP90C1 and CYP90D1 catalyse C-23 hydroxylation and that CYP90A needs to have its role in $\mathrm{BR}$ biosynthesis clarified.

The relatively high level of CYP90A1 expression has led to CPD promoter reporter gene fusions being extensively used to indicate where BRs are produced. CPD promoter:GUS fusion transformants indicate that this gene is expressed in roots, leaves and flowers, but the gene seems expressed at lower levels in inflorescence stems and siliques (Mathur et al., 1998; Shimada et al., 2003). Expression of the CYP90A1 gene is confined to cotyledons and leaf primordia in etiolated seedlings, and is detectable in expanding leaves in light-grown plants (Mathur et al., 1998). Its transcription is modulated by external signals such as light, and repressed by brassinosteroids. It also exhibits diurnal regulation (Bancos et al., 2006).

The T-DNA tagged dwarf4 (dwf4) mutant is defective in CYP90B1 that catalyzes C-22 hydroxylation in BR biosynthesis. $d w f 4$ mutants are very similar to $c p d$ mutants but exhibit a less severe phenotype (Azpiroz et al., 1998). Restoration of the dwf4 mutant 
phenotype using C-22 hydroxylated BR intermediates provided the first evidence that CYP90B1 was a C-22 hydroxylase (Choe et al., 1998; 2001). Endogenous BR levels in the $d w f 4$ mutant and CYP90B1 over-expressed transgenic plants, and feeding experiments using BR intermediates confirmed that CYP90B1 encodes a C-22 hydroxylase on the side chain of BRs (Choe et al., 2001). The azole inhibitor brassinazole (BRZ), which was generated to specifically inhibit BR synthesis, has been shown to bind to CYP90B and inhibit its activity (Asami et al., 2001). Triadimefon, an azole fungicide used in agriculture, also binds to CYP90B1 to suppress BR biosynthesis (Asami et al., 2003). CYP90B1 expression is highly regulated with the gene being expressed at very low levels, which suggested it as a rate-limiting step. This regulation is also tissue specific with an enhancer tagged line of $d w f 4$ exhibiting expression in the leaf margin (Reinhardt et al., 2007).

CYP90C1 was first associated with leaf development when rotundifolia3 (rot3) mutants were isolated (Kim et al., 1998). rot3 mutants lack normal elongation of leaves and floral organs, but have a normal root and shoot phenotype (Kim et al., 1998). CYP90C1 is expressed in all organs, leaves, stems, floral organs and roots, and in all leaf cell layers. Over-expression of CYP90C1 results in plants with longer leaves, petioles and floral organs. The rot3 phenotype was rescued to wild type when Castasterone (CS) or Brassinolide (BL) was applied, but not when upstream precursors were used (Kim et al., 2005c). Reduced levels of 6-deoxocastasterone and castasterone were found in the rot3 mutant compared with wild type, confirming this mutant was defective in BR synthesis (Kim et al., 2005c).

The cyp90c1/cyp90d1 double mutant is phenotypically equivalent to the cpd (cyp90a1) and dwf4 (cyp90b1) mutants and C-23 hydroxylated BRs can rescue this dwarfism. This suggested that both CYP90C1 and CYP90D1 function redundantly as C-23 hydroxylases. However, over-expression of CYP90D1 in the cpd (cyp90a1) mutant did not complement the BR-related dwarf phenotype (Kim et al., 2005c). The function of these P450s was clarified when recombinant CYP90C1 and CYP90D1 enzymes were expressed in the baculovirus insect cell expression system and were found to hydroxylate various BR intermediates at the C-23 position (Ohnishi et al., 2006a). The preferred conversion of certain intermediates led to a revised BR synthesis pathway and also indicates that the role of CYP90A1 and the biochemical lesion in the cpd mutant needs to be clarified (Ohnishi et al., 2006a; Bishop, 2007).

Very rapid and exciting advances have been made in determining how CYP90s and CYP85s are regulated by a negative feedback mechanism at the transcriptional level. Within two hours of BL treatment CYP90 and CYP85 family member transcripts are down-regulated by bioactive BRs (Figure 38) (Mathur et al., 1998; Bancos et al., 2002; Goda et al, 2002; Tanaka et al., 2005). This regulation requires a functional Brassinosteroid Insensitive 1 (BRI1) receptor because mutants defective in the BRI1 receptor lack feedback regulation, which results in abnormally high BRP450 transcript levels and BR accumulation (Choe et al., 2001; Bancos et al., 2002; Noguchi et al., 1999). Experiments with the CYP90A1 promoter from Arabidopsis have identified a specific BR-response element to which the BZR1 transcriptional regulator in BR signaling binds as a repressor (He et al., 2005). This BR-response element is also present in promoters of the other
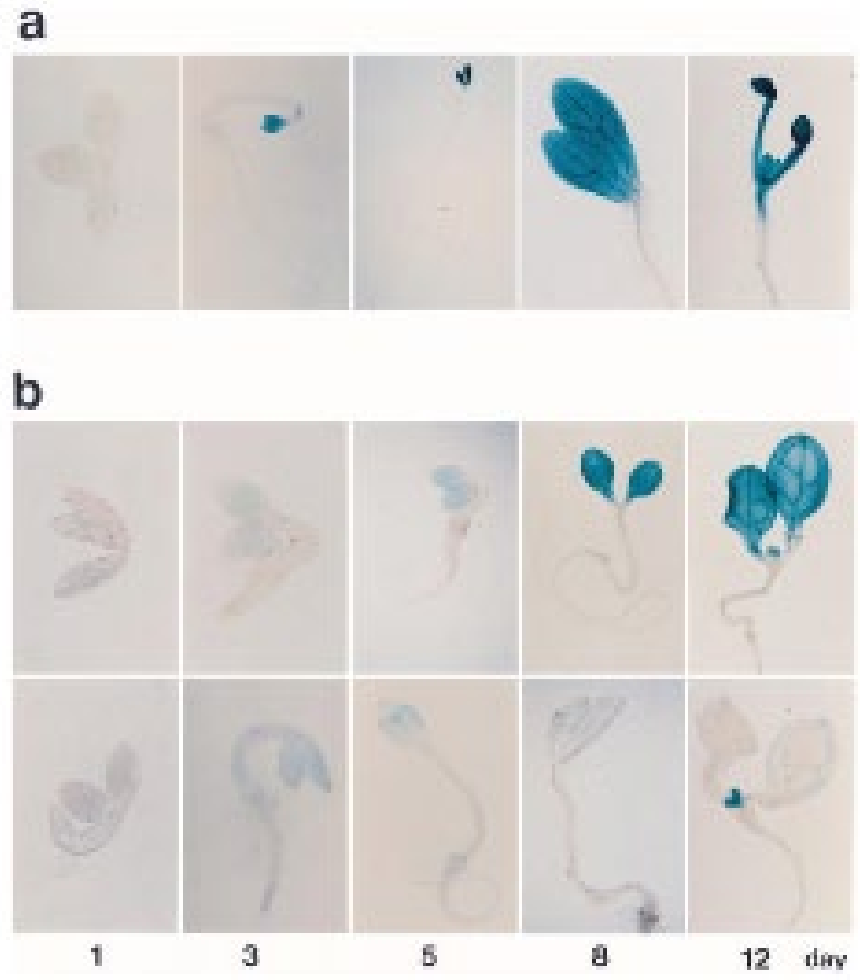

Figure 38. Feedback regulation of CYP90A1 promoter. Developmental activation and brassinolide-mediated inhibition of the CPD promoter during early seedling development.

GUS histochemical staining of transgenic seedlings carrying the CPD promoter-uidA reporter construct germinated and grown in the dark (a) and in the light (b). The lower panel in (b) shows seedlings grown in the presence of $1 \mu \mathrm{M}$ brassinolide. Reprinted from Mathur et al. (1998) with permission from Wiley-Blackwell Publishing.

$\mathrm{P} 450$ s in $\mathrm{BR}$ synthesis indicating coordinated repression of $\mathrm{BR}$ synthesis genes (He et al., 2005). The BR signaling pathway from BRI1 to BZR1 has now been elucidated and includes kinases and a phosphatase that leads to the altered phosphorylation status of BZR, with dephosphorylated BZR being present in the nucleus and exerting its effects (Kim et al., 2009b). Such coordinated regulation maintains BRs at optimal levels within any tissue.

\section{CYP91}

The genes previously classified as CYP91A1 and CYP91A2 have been renamed CYP81D1 and CYP81F1.

\section{CYP92}

The CYP92 family has apparently been lost in Arabidopsis and some other Brassicaceae, despite wide representation in the plant kingdom as early as in gymnosperms. With no clear evidence available, the in planta function of CYP92s remains in the dark. 


\section{CYP93}

There is a single representative of the CYP93 family in Arabidopsis. Several genes of this family described so far in other plant species are involved in biosynthesis of flavonoids, usually in the modification of the $\mathrm{C}_{2}-\mathrm{C}_{3}$ bond of ring $C$ (Ayabe and Akashi, 2006). The Arabidopsis CYP93D1 protein is however less than $50 \%$ identical to the other P450s of the CYP93 family, and its function might be different. CYP93E1, isolated from soybean was shown to hydroxylate triterpenes ( $\beta$-amyrin or sophoradiol) by heterologous expression in the yeast Saccharomyces cerevisiae (Shibuya et al., 2006). It is interesting to mention the physical clustering of the Arabidopsis CYP93D1 with CYP712B1 and that this gene association is preserved in several plant genomes (Nelson et al., 2008).

\section{CYP94}

CYP94s belong to the CYP86 clan of fatty acid-hydroxylases and share common motifs with fatty acid and alkane oxidizing enzymes from mammals and fungi. The genome of Arabidopsis contains three CYP94Bs, one CYP94C and three CYP94Ds including one pseudogene. The two CYP94D genes where initially classified as CYP86Ds.

CYP94C1 is the only characterized gene of the CYP94 family. In microsomes from recombinant yeast, CYP94C1 catalyzes the formation of $\omega$-hydroxyacids from fatty acids with varying chain lengths (C12-C16) and saturations (C18:1-C18:3) (Kandel et al., 2007). Furthermore, the production of dodecadioic acid from lauric acid was shown. Wounding and treatment with methyl jasmonate enhances the transcription of CYP94C1 in Arabidopsis. Both CYP94B1 and CYP94C1 were shown to be co-regulated with genes involved in jasmonate biosynthesis and signaling (Ehlting et al., 2008). The $\omega$-hydroxylase activity and transcriptional regulation in response to stress was also shown for CYP94s from other plant species (Benveniste et al., 2005; Le Bouquin et al., 2001; Tijet et al., 1998). A role of CYP94s in the oxylipin response can thus be proposed.

\section{CYP95}

The CYP95 family no longer exists. The original CYP95 sequence was based on a PCR fragment that had a frame shift in the I-helix region that caused it to be placed in a separate family CYP95. Isolation of the final full sequence from maize corrected this initial misnaming. CYP95A1 from maize was renamed CYP72A5.

\section{CYP96}

CYP96 seems to be a family unique to Angiosperms. In Arabidopsis it comprises 15 members, of which only one (CYP96A15) has been functionally characterized (Greer et al., 2007). CYP96A15 was identified as a candidate for the midchain alkane hydroxylase (MAH) of the surface wax biosynthesis pathway based on preferential gene expression in stem epidermis and for belonging to the CYP86 clan, which contains several families involved in fatty acyl hydroxylation. Analysis of surface waxes in stems of CYP96A15 insertional mutant lines (mah1-1 to mah1-3) showed absence or much reduced levels of secondary alcohols and ketones, indicating CYP96A15 indeed encoded the midchain alkane hydroxylase of wax biosynthesis (Figure 39). This was confirmed by ectopic over-expression of CYP96A15/MAH1 under the CaMV35S promoter, which resulted in accumulation of secondary alcohols in Arabidopsis leaf wax (only traces of these compounds are normally found in wild-type leaves). Ketones were also produced, suggesting that CYP96A15 also catalyzed the subsequent conversion of secondary alcohols to ketones (Figure 39). CYP96A15 was found to be mostly expressed in epidermal pavement cells, but not in trichomes and guard cells (Figure 40). CYP96A15green fluorescent protein fusion localized to the ER, indicating that the secondary alcohol and ketone wax products must be transported from the ER to the plasma membrane for export outside the epidermal cells.

\section{CYP97}

The plant-specific CYP97 family consists of three distinct subfamilies that emerged before the higher plant/green algae split. It is represented ubiquitously in Arabidopsis and all other land plants for which full genomes are available with single candidates in each subfamily CYP97A, CYP97B and CYP97C. Of the three, CYP97B represents the oldest of these three as it shares the highest phylogenetic identity with the CYP97E and CYP97F sequences of diatoms and other protists (Nelson et al., 2008).

Based on the finding that the transcript of CYP97B3 showed circadian phasing similar to the phasing of CYP97A3 and CYP97C1, it was suggested that the transcriptional regulation of the three CYP97 genes was shared (Pan et al., 2009). Kim and coworkers (2010) hypothesised that apparent similar developmental expression patterns, with highest expression in Arabidopsis leaves and seedlings, is consistent with overlapping and cooperative functions for all three hydroxylases. Not surprisingly, several putative cis-acting elements overrepresented in the carotenoid intermediate and core pathway were detected in the promoters of CYP97A3, CYP97C1 and CYP97B3 (Pan et al., 2009). In silico prediction of subcellular localisation indicated co-localisation of the CYP97 family proteins in chloroplasts (Schuler et al., 2006), which was recently experimentally confirmed by global analysis of the chloroplast proteome (Zybailov et al., 2008).

On the basis of genetic evidence, using knock-out mutants of Arabidopsis, CYP97A3 and CYP97C1 were shown to hydroxylate the $\alpha$-carotene $\varepsilon$-ring and the $\beta$-ring of $\alpha$-carotene and $\beta$-carotene, respectively (Figure 41) (Tian et al., 2004; Kim and DellaPenna, 2006). Consistent with the finding that residual accumulation of hydroxylated carotenoids was detected in mutants lacking activity of the CYP97A3 and CYP97C1 (Fiore et al., 2006; Kim and DellaPenna, 2006), a role for CYP97B3 was suggested in a-carotene modification (Kim et al., 2010). Complementary to the previous analyses of knock-out mutants, Kim and coworkers analysed carotenoid accumulation in Arabidopsis lines overexpressing the three P450 carotenoid hydroxylases (Kim et al., 2010). Even though over-expression of the individual hydroxylases had only a modest impact on carotenoid metabolic flux, this study provides evidence supporting dual activities of carotenoid hydroxylases, with functions in both the $\alpha$ - and $\beta$-carotene pathways and a potential function of CYP97B3 as a $\beta$-ring hydroxylase. 


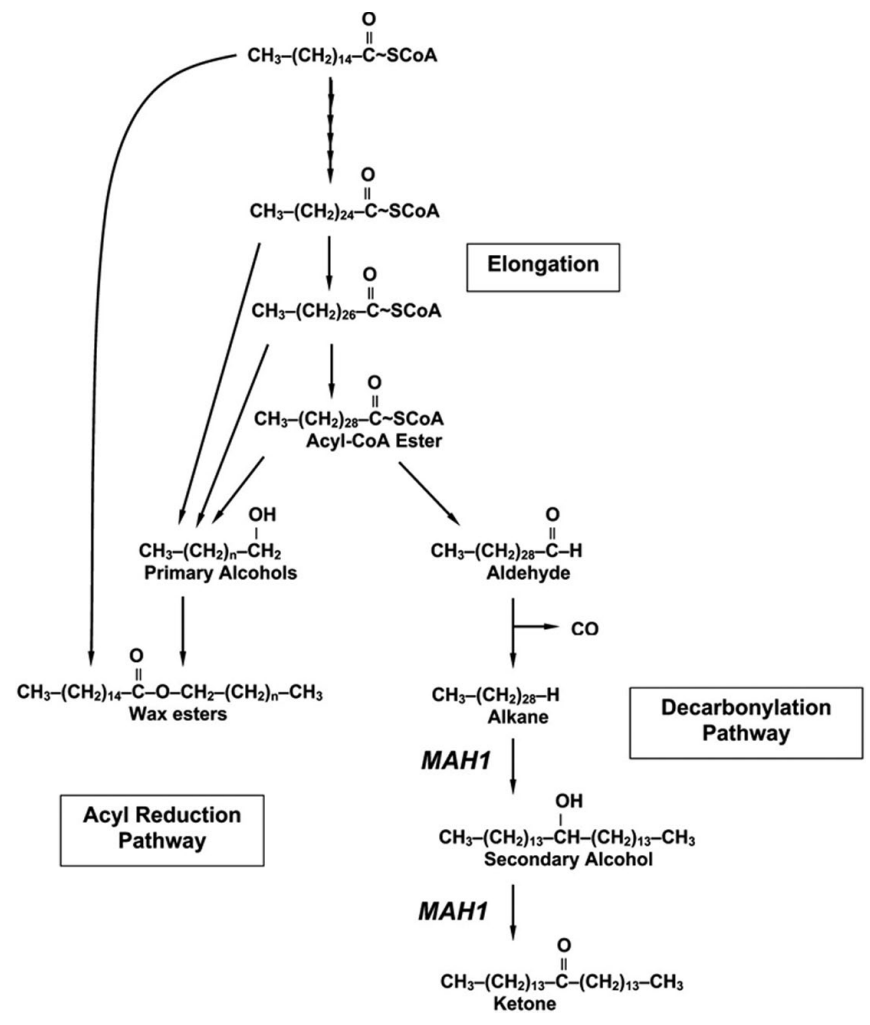

Figure 39. Proposed action of CYP96A15/MAH1 in the decarbonylation pathway of wax biosynthesis. Reprinted from Greer et al. (2007).

Only the reactions modifying 30:0 acyl CoA are shown.
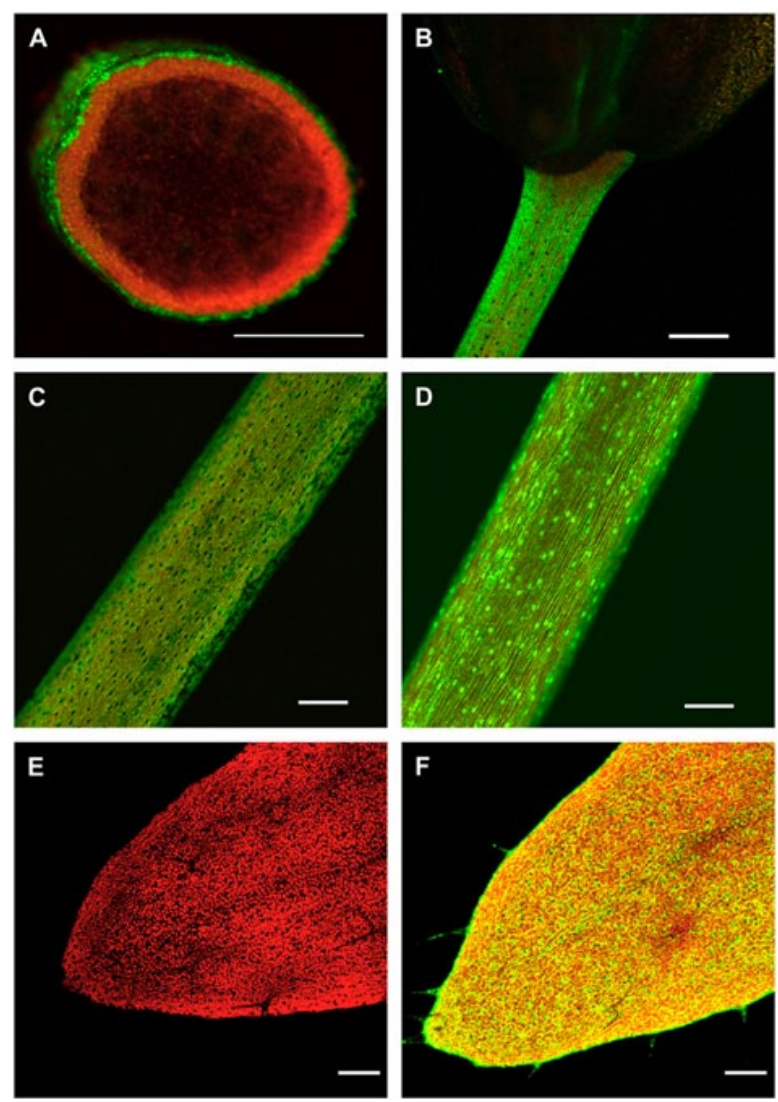

Figure 40. Expression of CYP96A15/MAH1 (Greer et al., 2007).

MAH1:GFP fusion protein was expressed in the mah1-1 mutant background under the control of the native $M A H 1$ promoter (A, B, C, E) or the 35 S promoter (D, F). (A)(D) stems, (B) petioles, (C) sepals, (E) and (F) leaves. Autofluorescence is depicted as red and images are layered signals (autofluorescence and GFP).

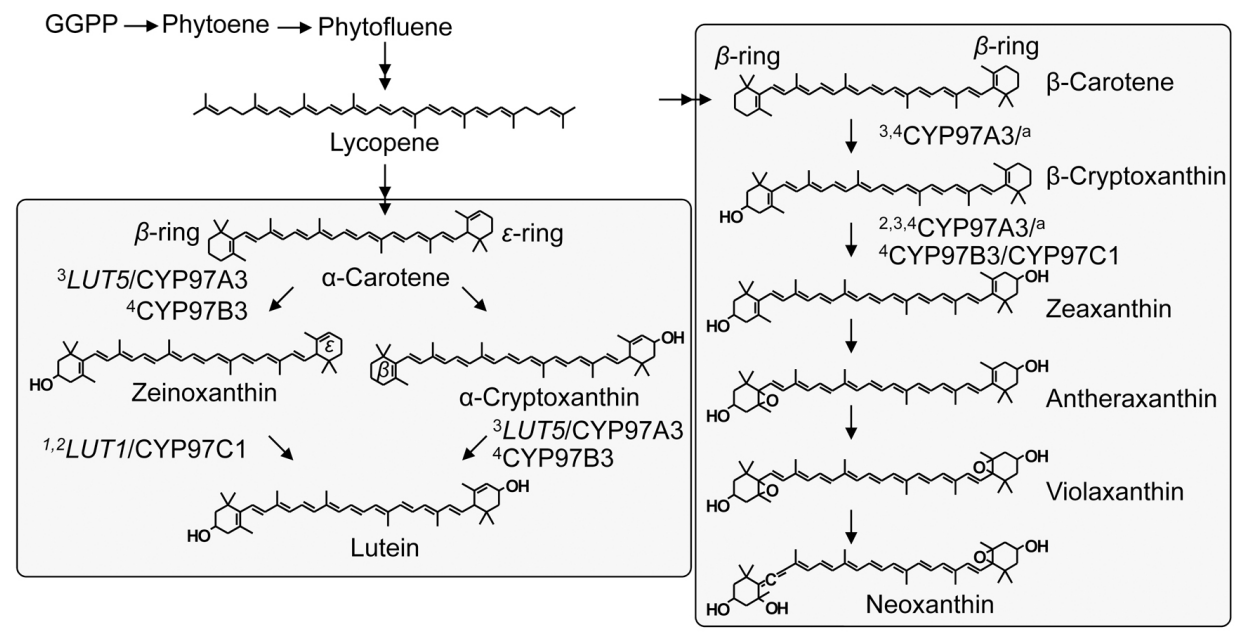

Figure 41. Schematic carotenoid biosynthesis pathways: the alpha- and beta-carotenoid routes to the major metabolites lutein and neoxanthin.

Activity of P450 enzymes (CYP97A3, CYP97B3, CYP97C1), deduced from mutant phenotype analysis, heterologously expressed enzymes or biochemical analysis of over-expression lines are indicated at the pathway step ( ${ }^{1}$ Tian et al. (2004); ${ }^{2}$ Fiore at al. (2006); ${ }^{3} \mathrm{Kim}$ and DellaPenna (2006); ${ }^{4} \mathrm{Kim}$ et al. (2010)). anon-heme $\beta$-carotene-ring hydroxylase. 

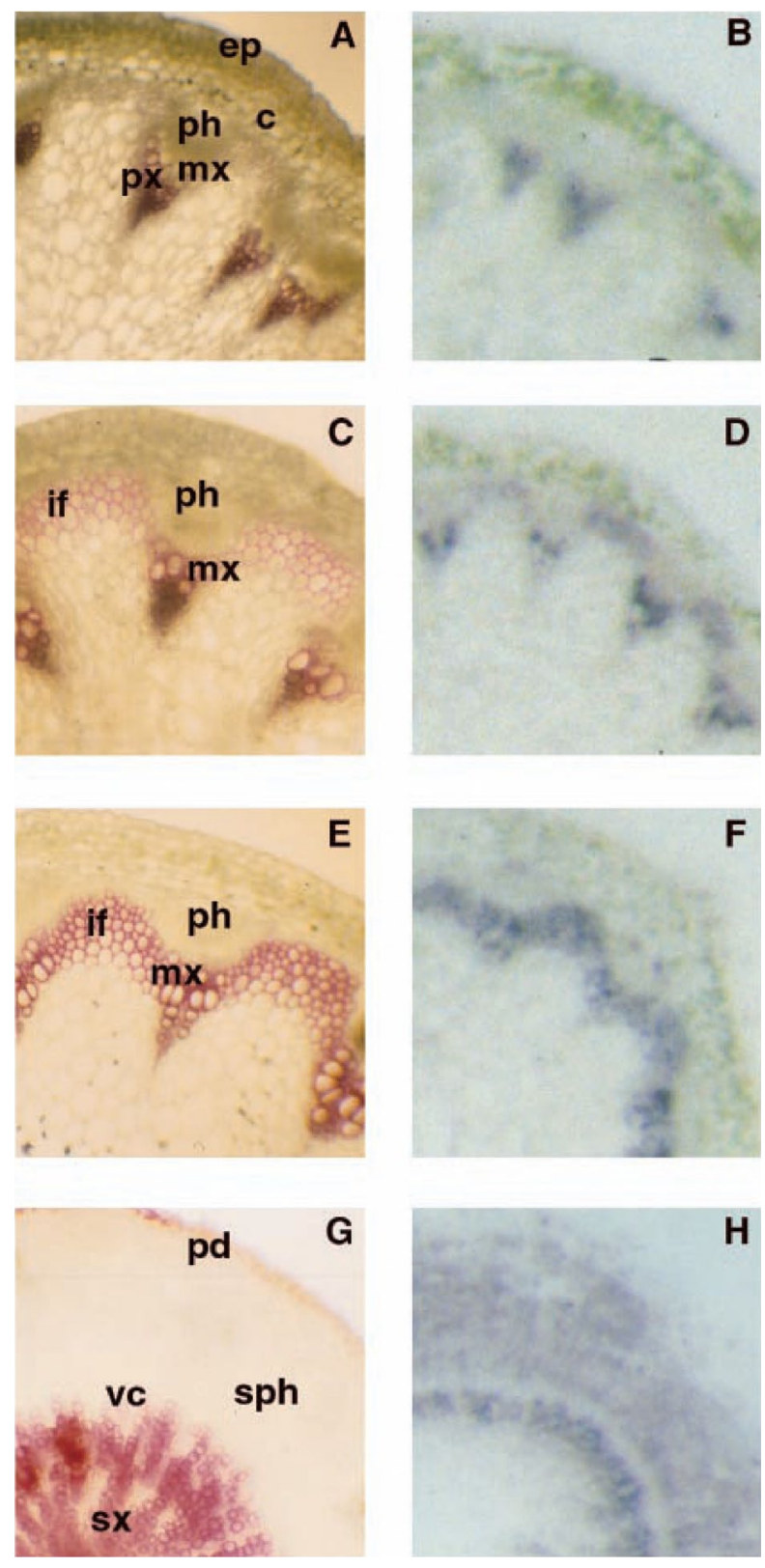

Figure 42. Immunolocalization of the expression of CYP98A3 in stems and roots.

Hand-cut transversal sections of inflorescence stems and roots were stained with phloroglucinol $\mathrm{HCl}$, a red coloration reflecting lignin content. Adjacent sections were printed onto nitrocellulose and revealed using anti-CYP98A3 polyclonal antibodies. Blue staining is indicative of CYP98A3 expression. In stems, prints were taken at increasing distances from the apical meristem to monitor temporal and developmental expression of CYP98A3 in conjunction with the differentiation of lignified tissues. No blue staining was obtained with preimmune antibodies. (A,C,E), and (G), lignin staining with phloroglucinol; $(B, D, F)$, and $(H)$, immunostaining of CYP98A3. (A) and (B), upper segment of the stem, close to the flower bud; (C) and (D), mid-stem; (E) and (F), lower, well differentiated stem close to the rosette; $(\mathbf{G})$ and $(\mathbf{H})$, root. ep, epidermis; $c$, cortex; $p x$, protoxylem; $m x$, metaxylem; $p h$, phloem; if, interfascicular region; sx, secondary xylem; $v c$, vascular cambium; sph, secondary phloem; $p d$, periderm. Reprinted from Schoch et al. (2001) with permission from American Society for Biochemistry and Molecular Biology.

\section{CYP98}

CYP98 is a low gene copy family belonging to the CYP71 clan. There are three CYP98 genes in Arabidopsis. CYP98A3 is present in single copy on chromosome 2. The high frequency of CYP98A3related ESTs found in many plant species, especially in lignifying tissues, initially drew attention to this gene. CYP98A3 was found to be expressed in all organs of Arabidopsis, but at higher levels in inflorescence stems, roots and siliques (Schoch et al., 2001). Immunolocalization of the protein on tissue prints indicated that its expression was confined to tissues undergoing active lignification in stems and roots (Figure 42). The site and high expression level of CYP98A3 thus suggested its potential involvement in the 3-hydroxylation of $p$-coumaric acid. To test this hypothesis, CYP98A3 was expressed in yeast. Yeast-expressed CYP98A3 did not metabolize free $p$-coumaric acid, nor its CoA or glucose conjugates, but was found to very actively hydroxylate the shikimimate and quinate esters of $p$-coumaric acid in the 3 position of the phenolic ring (Figure 43). Isomers of the substrates were also converted. The products of CYP98A3 metabolism could be converted back into CoA esters before being methylated by caffeyl CoA O-methyltransferase. CYP98A3 was thus capable of catalyzing the conversion of $p$-coumaroyl quinate into chlorogenic acid, and also expected to catalyze the meta-hydroxylation step for the formation of lignin monomers. This was confirmed by an independent genetic approach based on an EMS mutant collection selected on the basis of reduced epidermal fluorescence. The ref8 mutants, defective in the accumulation of sinapoyl malate in rosette leaves, were shown unable to synthesize caffeoyl residues and downstream compounds by feeding radiolabelled precursors (Franke et al., 2002a). ref8 had a mutation modifying the L-helix of CYP98A3 that was successfully complemented with the wild-type gene. The mutant was severely affected in its development and essentially female sterile (Figure 44). Metabolic profiling of ref8 indicated that it accumulates $p$-coumarate esters instead of sinapoylmalate (Franke et al., 2002b). It also deposited a lignin primarily formed from $p$-coumaroyl alcohol monomers that resulted in increased lignin degradability, vascular bundle collapse, and increased susceptibility to fungal attack. Altogether, CYP98A3 thus appeared as a $p$-coumaroyl ester hydroxylase involved in biosynthesis of lignin monomers and soluble phenolics, a demonstration that challenged the classical routes so far described in phenylpropanoid metabolism (for more on this pathway see also Fraser and Chapple (2011).

More in-depth investigations of the impact of the ref8 mutation on lignified tissue, plant morphology and composition essentially confirmed these conclusions (Patten et al., 2010). They were also confirmed by the isolation of a T-DNA mutant in the Ws background and by obtaining co-suppressed lines (Abdulrazzak et al., 2005). The severity of the growth inhibition phenotype was found to be correlated with the degree of co-suppression, with an extremely severe phenotype in the T-DNA insertion mutant. Decrease in CYP98A3 gene expression was associated with limp stems and inflorescences, bushy appearance, accumulation of flavonoid glucosides and anthocyanins, male sterility and an ectopic lignification phenotype (Figure 45). While starting to bolt, T-DNA mutants did not develop fertile flowers and stems did not exceeded $3 \mathrm{~cm}$. Reduced cell growth was associated with an alteration in cell wall polysaccharide, decrease in crystalline 
<smiles>CC(C)CC(=O)O</smiles>

trans-(p-coumaroyl)-shikimic acid

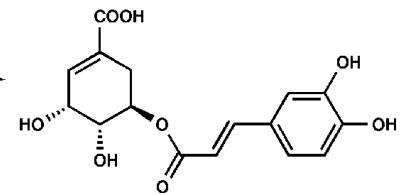

trans-( $p$-caffeoyl)-shikimic acid

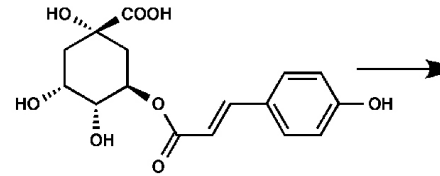

trans-( -coumaroyl)-quinic acid

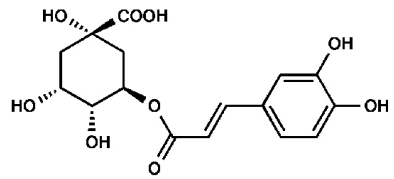

Chlorogenic acid
Figure 43. The reactions catalyzed by CYP98A3.

5-O-(4-Coumaryol)-shikimate is metabolized 4 times more efficiently than 5-O-(4-coumaryol)-D-quinate and is probably the favored substrate in vivo.

cellulose and profound modification in gene expression reminiscent of stress response. Reduced growth was partially restored by exogenously added $p$-coumaroyl shikimate but not caffeic or chlorogenic acid. In this study, in despite of a strong reduction in lignin content and accumulation of hydroxycinnamoyl units in the null mutant, significant amounts of guayacyl and syringyl residues were still detected, in particular in the roots, and the purple coloration of the cauline leaves was found to be related to the accumulation of susbtantial amounts of sinapoylated anthocyanins.

CYP98A8 and CYP98A9 are clustered on chromosome 1. They are intronless, while CYP98A3 is interrupted by two introns, one of them, the "M" intron, is highly conserved in the CYP71 clan of P450 genes (Paquette et al., 2000). The CYP98A8 and CYP98A9 proteins are $69 \%$ identical and each of them only about $50 \%$ identical to CYP98A3. In classical phylogenetic reconstructions, based on protein sequence comparison, they do not belong to the monophyletic clade that includes the confirmed $p$-coumaroyl ester hydroxylases from all Angiosperms. This was puzzling considering that only a single CYP98A8 ortholog is found in the Brassica napus genome (Figure 46). It led Matsuno et al. (2009) to examine the cDNA phylogeny and rates of codon substitution within the CYP98 family. They concluded from this study that CYP98A8 and CYP98A9 result from the accelerated evolution of an ancestral CYP98A duplicate in Brassicaceae or in one of their close ancestors. The absence of introns and the expression of CYP98A8 and CYP98A9 in the germinal cells support the hypothesis that this duplication occurred via mRNA-mediated transposition (retroposition). Amino acids with a high posterior probability of positive selection were then mapped on a three-dimensional model of the enzyme. They clustered in the distal cavity of the active site and in two regions at the surface of the protein. Resulting modifications predicted an increased size of the active site and enzyme flexibility, possibly increased accessibility of the substrate pocket and most likely modified membrane interaction. The expression profiles of CYP98A8/A9, based on in silico transcriptome analysis and imaging of promoter:GUS fusions indicated a main function related to floral development, in the an-

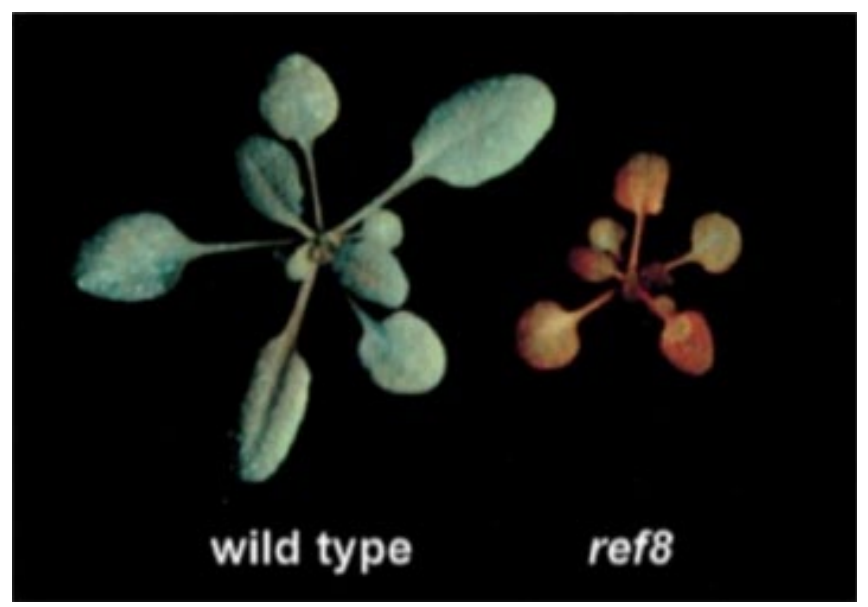

Figure 44. Phenotype of the ref8 mutant.

UV and size phenotypes of wild type and ref8, a CYP98A3 null mutant. Rosette leaves of 3-week-old plants were photographed under $365 \mathrm{~nm}$ UV light using a yellow barrier filter. The blue-green color of the wild-type rosettes is due to the fluorescence of sinapoylmalate. The red fluorescence of the ref8 plant is due to chlorophyll fluorescence that is revealed in the absence of sinapate ester fluorescence. Reprinted from Franke et al. (2002a) with permission from Wiley-Blackwell Publishing.
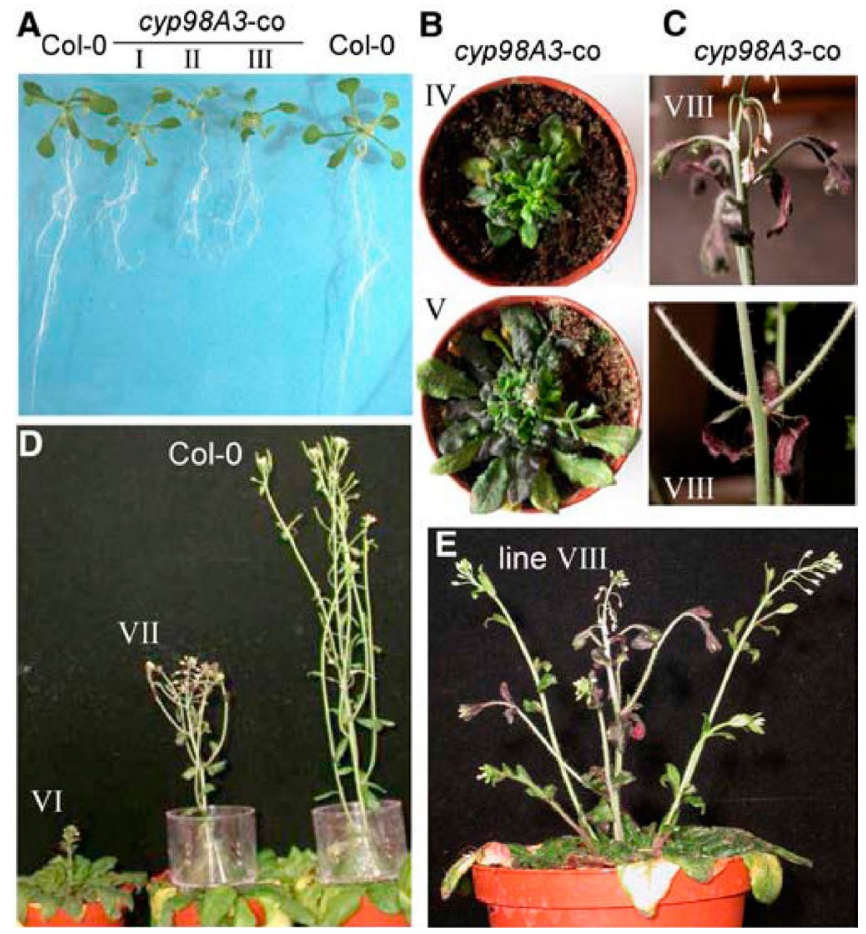

Figure 45. Phenotypes associated with CYP98 co-suppression.

Arabidopsis Col-0 plants were transformed with a CaMV 35S:CYP98A3 construct (Abdulrazzak et al., 2006). Approximately $10 \%$ of primary transformants showed cosuppression of CYP98A3. The phenotypes of selected transformants are shown in A to $E$ (roman numbers identify individual transformants). (A) Three-weeks old T1 plants grown on vertical agar plates. Wildtype (Col-0) plants are shown on both sides. (B) to (E), Different 10-week-old cosuppressed T1 lines grown on soil (B). Moderately cosuppressed plants arrested at different stages of bolting stem development (C-E). When plants bolted, inflorescences showed purple stems and cauline leaves; they were limp, in most cases male sterile, and rarely produced viable seeds (C) and (E). 


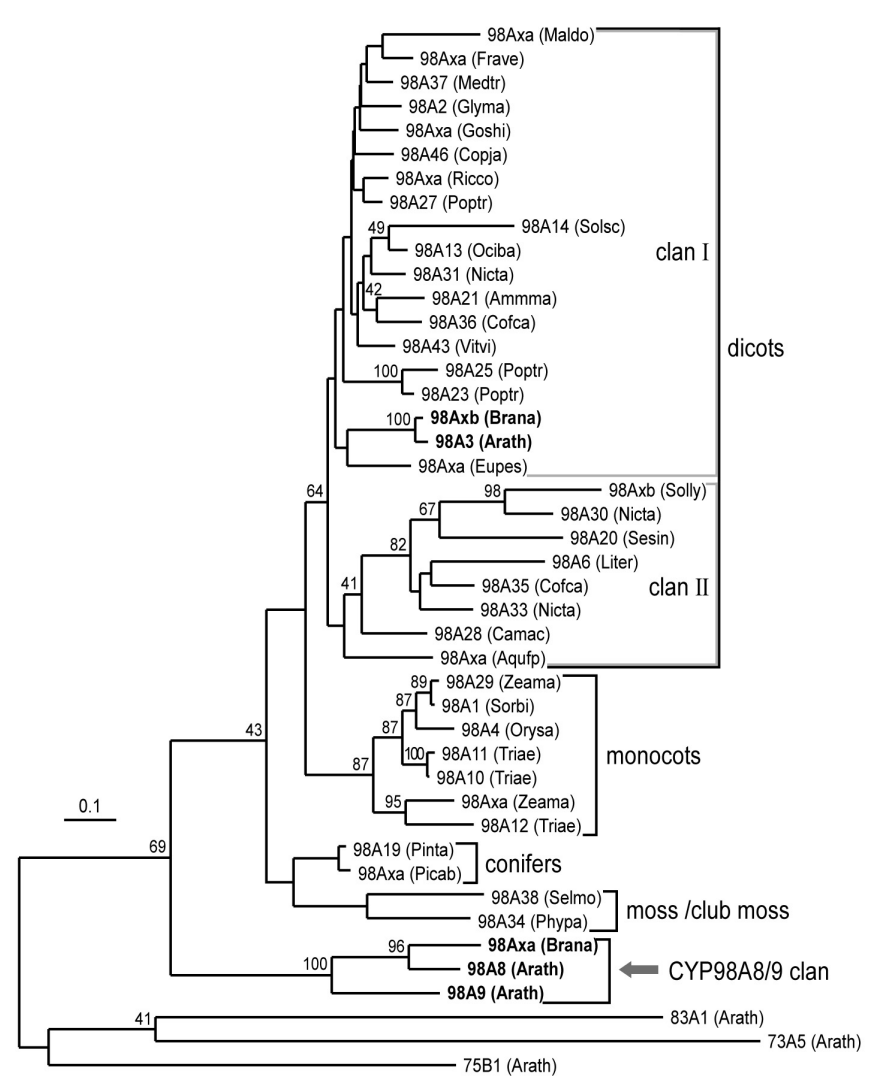

Figure 46. Phylogeny of the CYP98 proteins

thers and in particular in the tapetal cells. Some expression in the leaf and root vasculature and root tip was however observed for CYP98A9. Previous large-scale gene co-expression analysis had predicted the involvement of CYP98A8 and CYP98A9 in a parallel phenolic pathway active in the flowers of $A$. thaliana (Ehlting et al., 2008). Metabolic profiling of young inflorescences of the T-DNA insertion mutant of CYP98A8, of a RNAi mutant of CYP98A9, and of the double mutant fully confirmed this assumption and revealed that while both CYP98A8 and CYP98A9 share the ability to catalyze the triple meta-hydroxylation of $N^{1}, N^{5}, N^{10}$ tricoumaroyl spermidine, only CYP98A8 is able to catalyze the second meta-hydroxylation of the three phenolic rings to convert $N^{1}, N^{5}, N^{10}$-triferuloylspermidine into $N^{1}, N^{5}, N^{10}$-trihydroxyferuloyl spermidine (Figure 47) (Matsuno et al., 2009). The final product of this pathway, $N^{1}, N^{5}$-dihydroxyferuloyl- $N^{10}$-sinapoyl spermidine is a major constituent of the pollen coat and possibly contributes to the pollen wall structure. The catalytic functions of both CYP98A8 and CYP98A9 have been confirmed after recombinant expression in yeast. This alternative phenolic pathway offers the opportunity for the formation of polyamine-conjugated syringyl and sinapoyl units, and provides the first documented example of evolution of new function via retroposition in plants (Figure 48). Suppression of this pathway leads to a surprisingly minor defect in pollen development and fertility as also stressed by Fellenberg et al. (2009). Also guided by microarray gene co-expression,

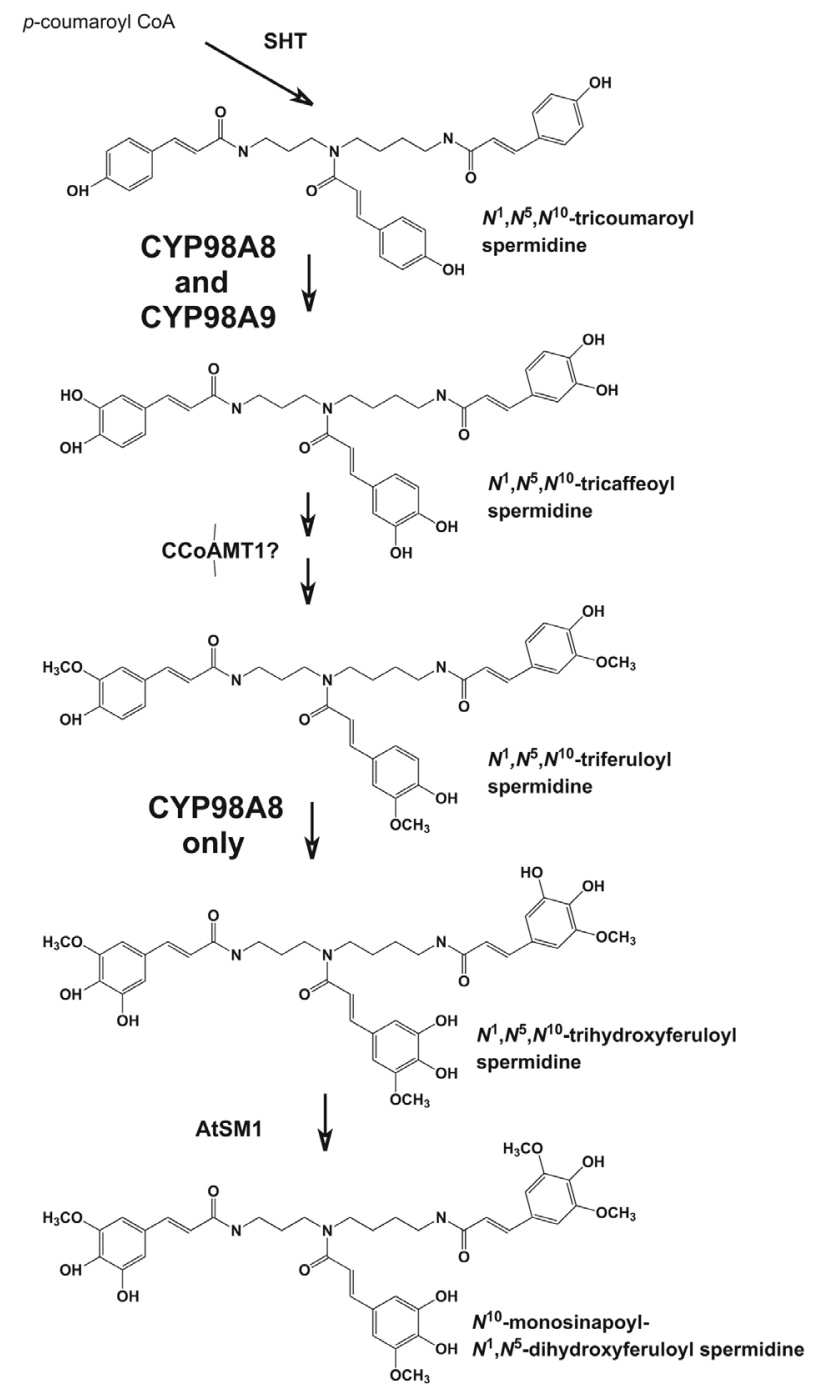

Figure 47. The reactions catalyzed by CYP98A8 and CYP98A9 in the phenolamide pathway and formation of major pollen coat constituents.

SHT : spermidine hydroxycinnamoyl transferase

AtSM1 : cation-dependent O-methyltransferase-like protein CCoAMT1: caffeoyl CoA O-methyltransferase 1

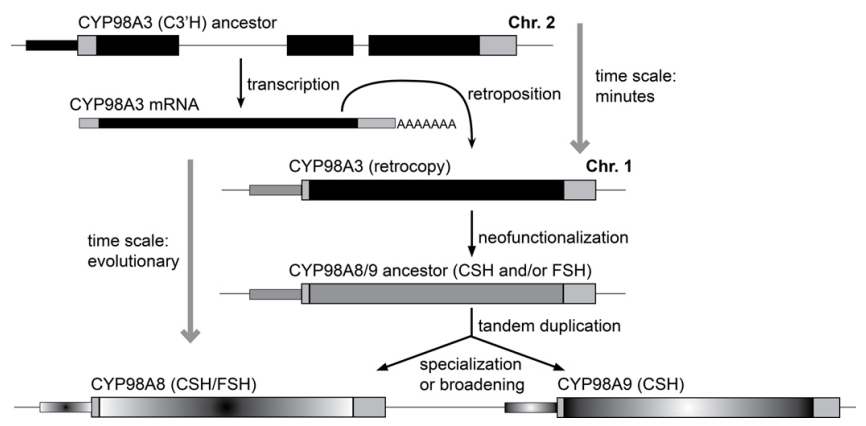

Figure 48. Evolution of CYP98A8 and CYP98A9. 
these authors confirmed the flower bud-specific co-expression of CYP98A8 with other genes in the pathway. They provide an extensive description of the metabolic profile of the T-DNA insertion cyp98a8 mutant and evidence of a suppression of the transcripts coding for the other enzymes in the pathway in cyp98a8 plants. This suggests a possible negative feeback regulation in this tapetum-specific pathway.

\section{CYP99}

The CYP99 family belongs to the CYP71 clan and is represented only in grasses within Monocots. It has been associated with the synthesis of the diterpenes momilactones in rice (Shimura et al., 2007).

\section{CYP701}

CYP701s appeared very early in the evolution of land plants; they are found in moss (Physcomitrella). They have not so far been reported in liverworts where CYP88 is represented, but may be found after complete sequencing of the Marchantia genome (Nelson and Werck-Reichhart, 2011). Like CYP88s (see the CYP88 section) they are involved in the biosynthesis of GAs. There are, however, striking differences between this P450 family and the CYP88s.

Contrary to most other oxygenases in terpenoid hormone biosynthesis, CYP701s belong to the CYP71 clan. The evolutionary lineage of CYP701 and CYP88s is thus very different even though they carry out similar and successive enzymatic reactions: CYP701A3 encodes the ent-kaurene oxidase (KO) carrying out three oxygenation steps in the conversion of ent-kaurene to ent-kaurenoic acid and ent-kaurenoic acid is then the substrate for the CYP88 enzymes (see Figure 37).

CYP701A3 is the only member of the CYP701 family found in Arabidopsis. CYP701A3 was cloned through map-based cloning of the Ga3 gene in Arabidopsis. ga3 mutants were identified as non-germinating, gibberellin-responsive dwarfs following EMS mutagenesis (Koornneef and Vanderveen, 1980). Similar to the dwarf BR mutants, the phenotype of these recessive mutants highlights the role of gibberellins in plant development. Both ent-kaurenoic acid and GA are able to rescue the dwarf phenotype and ent-kaurene accumulates in the mutants. These observations indicate that ga3 mutants are blocked in the conversion of ent-kaurene to ent-kaurenoic acid (Figure 37) (Helliwell et al., 1998). This conversion was known very early on to be catalyzed by a P450 enzyme (Murphy and West, 1969) and can be inhibited by compounds with structural similarity to entkaurene, e.g. the growth-retardant entantiomers of paclobutrazole or tetcyclasis possessing $\mathrm{N}$-containing heterocycles. Confirmation of the role of GA3 was carried out by sequencing various mutant alleles at this locus and by the rescue of the ga3 mutant phenotype through genetic complementation using a genomic clone (Figure 49). The CYP701A3 coding sequence was then expressed in yeast to confirm the function of the protein in catalyzing the conversion of ent-kaurene to ent-kaurenoic acid (Helliwell et al., 1999).

The CYP701A3 gene is expressed in young, fast growing seedlings (Helliwell et al., 1998). In older plants, expression is detected in leaves, but is higher in elongating stems and inflo-

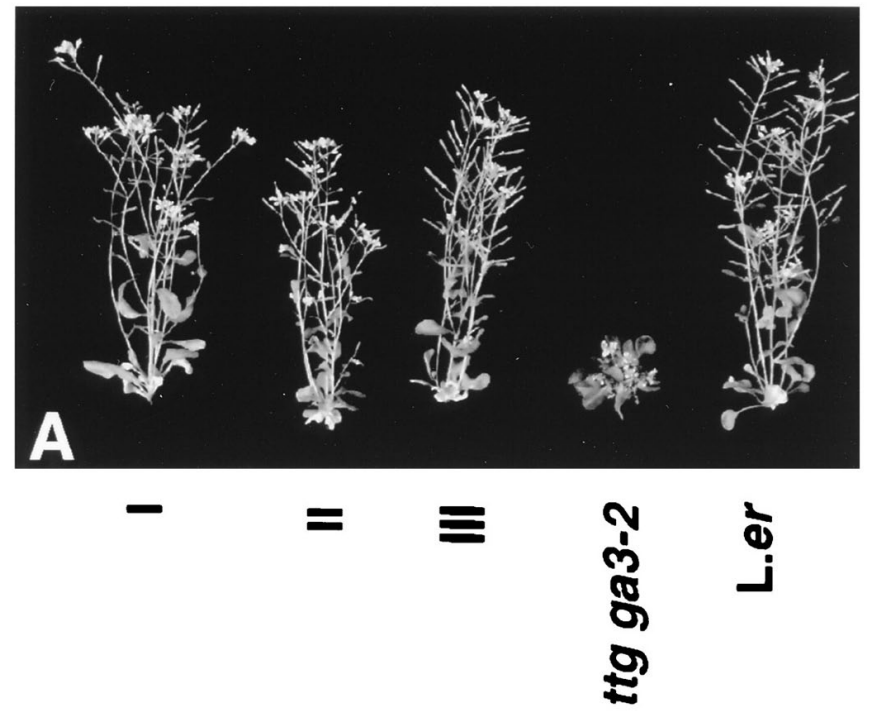

Figure 49. The ga3 mutant phenotype and complementation of the mutation with CYP701A3.

(I-III) Three kanamycin resistant plants from the transformation of ga32 with a genomic CYP701A3 clone. (ttg ga3-2): The mutant ga3-2 line. (L.er): The wild-type Landsberg erecta line. Reprinted from Helliwell et al. (1998) with permission from the National Academy of Sciences.

rescences. The gene does not seem to be subject to feedback inhibition. Over-expression of CYP701A3 leads to plants that are more resistant to the $\mathrm{P} 450$ inhibitor paclobutrazole (Swain et al., 2005).

CYP701A3 has a predicted chloroplast transit peptide with a cleavage sequence at amino acid 28-29 from the N-terminus (Helliwell et al., 2001b). GFP-tagging experiments have shown that this P450 is targeted to the outer surface of chloroplasts on the plastid envelope, whereas CYP88s are located on the ER (Figure 50). CYP701A3 therefore provides a link between the early biosynthesis of GAs in the chloroplast and later reactions on the endoplasmic reticulum and in the cytosol. The example of the CYP701/CYP88 couple in the biosynthesis of GAs shows that the general rule that P450s involved in the same pathway or catalyzing similar reactions are phylogenetically close is not always true, and that genes in the same pathway can be recruited from presently distant families.

\section{CYP702}

There are six CYP702 genes and two pseudogenes annotated in the Arabidopsis genome, most of them as tandem repeats on chromosome 4. They belong to the CYP85 clan and seemingly diverged recently in Brassicaceae (Nelson and Werck-Reichhart, 2011), from an ancestral CYP87. They are expected to be involved in triterpenoid metabolism based on physical clustering and gene co-expression with the members of the CYP705/CYP708 families that seem to share a common evolutionary history (Field and Osbourn, 2008; http://www-ibmp.u-strasbg.fr/ CYPedia/). 


\section{CYP703}

A single CYP703 gene is annotated in Arabidopsis as in the majority of vascular plant genomes (Nelson and Werck-Reichhart, 2011). CYP703 genes are also found in non-vascular plants. Three copies were identified in the genome of $P$. patens, but the CYP703 family is not represented in green algae. ESTs encoding CYP703 orthologs are usually found in spores or reproductive (male) tissues in all plant species (Morant et al., 2007). This suggests an essential function for CYP703 enzymes common to all land plants.

To investigate this function, Morant et al. (2007) started to precisely examine the expression profile of CYP703A2 from Arabidopsis, thus showing that transcripts were detected in closed flower buds, but not in any other plant tissues. Profiling of Prom $_{\text {СРР70зA2: }}$ :GUS transformants showed that expression was confined to the anthers and more specifically to the tapetum and the microspores from the tetrad stage to the lysis of the tapetum.

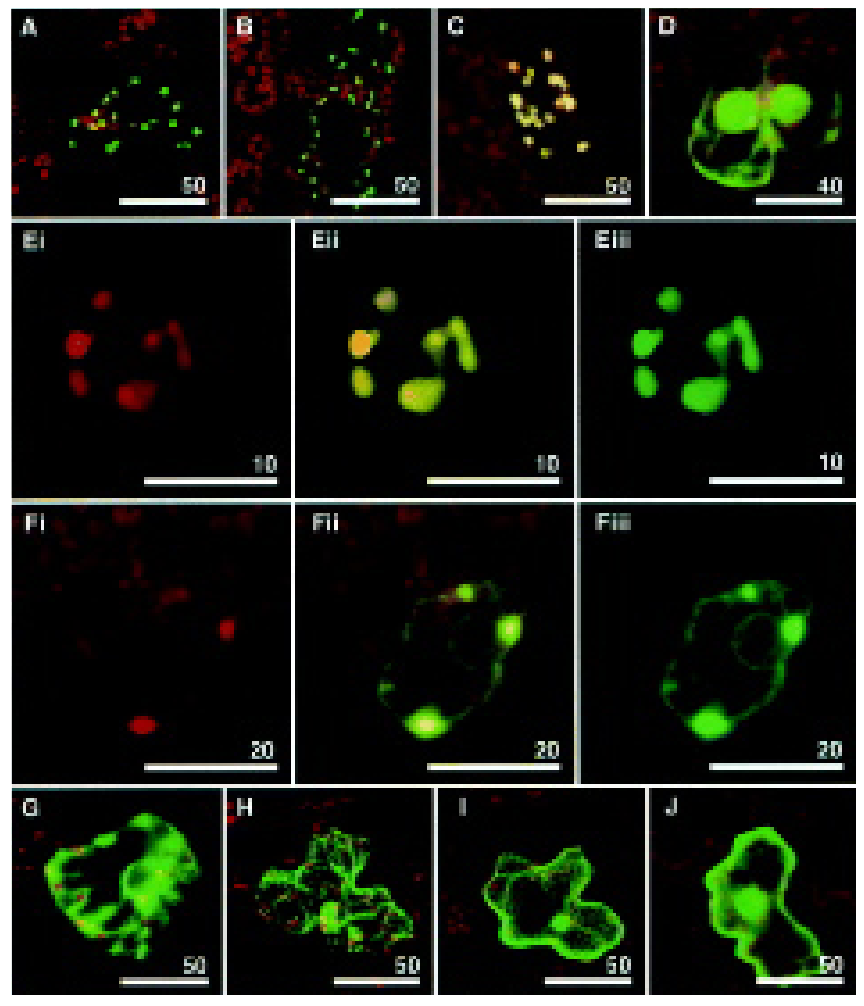

Figure 50. Confocal images of tobacco leaf cells following microprojectile bombardment showing chloroplast localization of CYP701A3.

Images were taken $24 \mathrm{~h}$ after bombardment of tobacco leaves with tungsten particles coated with individual plasmid constructs. The cells expressing the GFP construct are epidermal cells with the exception of $\mathrm{Ei}-\mathrm{iii}$ which show a mesophyll cell. The images are dual GFP (green) and chlorophyll (red) channels, except those labelled (i) and (iii) which show separate chlorophyll and GFP channels, respectively. The lengths of scale bars are given in $\mu \mathrm{m}$ in each panel. (A) TPCPS-GFP (copalyl diphosphate synthase transit peptide-GFP fusion: plastidial control), (B) TPKS-GFP (kaurene synthase transit peptide-GFP fusion: plastidial control), (C) RbcS-GFP, (D) smGFP, (E) TPCYP701A3-GFP, (F) CYP701A3-GFP, (G) TPCYP88A3-GFP, (H) TPCYP88A4-GFP, (I) mGFP5, (J) 20ox2-GFP. Images reprinted from Heliwell et al. (2001) with permission from Wiley-Blackwell.
Examination of two independent T-DNA insertion mutants revealed identical phenotypes, including longer blooming inflorescences, reduced filament growth, and pollen abortion with a resulting reduced number of elongated siliques and seed set. Interestingly, the phenotype varied along the inflorescence stem and was more severe at the base than at the top of the older inflorescence, but in older inflorescences the amount of pollen was still reduced. In cyp703a2 lines, the pollen surface appeared completely smooth compared to the ridged surface of the wild type when examined by scanning electron microscopy and was devoid of the fluorescent layer due to phenolamides of the pollen coat (Figure 51) (see CYP98 section). A significant proportion of the pollen grains appeared aborted at the early stages of their development. Mature cyp703a2 pollen grains, but not wild type pollen grains, collapsed when a vacuum was applied in preparation for electron microscopy, this reduction in physical resistance was due to a complete absence of exine. Cross-pollination of cyp703a2 mutants with wild-type pollen fully restored fertility.

When expressed in yeast, CYP703A2 was found to catalyze the in-chain monohydroxylation of saturated $C 10: 0$ to $C 16: 0$ fatty acids, capric (C10) and lauric (C12) fatty acids being the best substrates. Hydroxylation always occurred on carbons $6,7,8$ or 9 (but with a preference for carbon 7), at a constrained distance from the carboxylic acid (Figure 52). Incubation of the recombinant enzyme with an Arabidopsis anther lipid extract, corroborated that lauric acid might be an in vivo substrate of the enzyme. The main constituent of exine is sporopollenin which is also found in moss and fern spore walls. Since it is highly resistant to chemical degradation, its definitive composition and structure is not established, although sporopollenin is usually described as a polymer of long saturated chains incorporating varying amounts of aro-

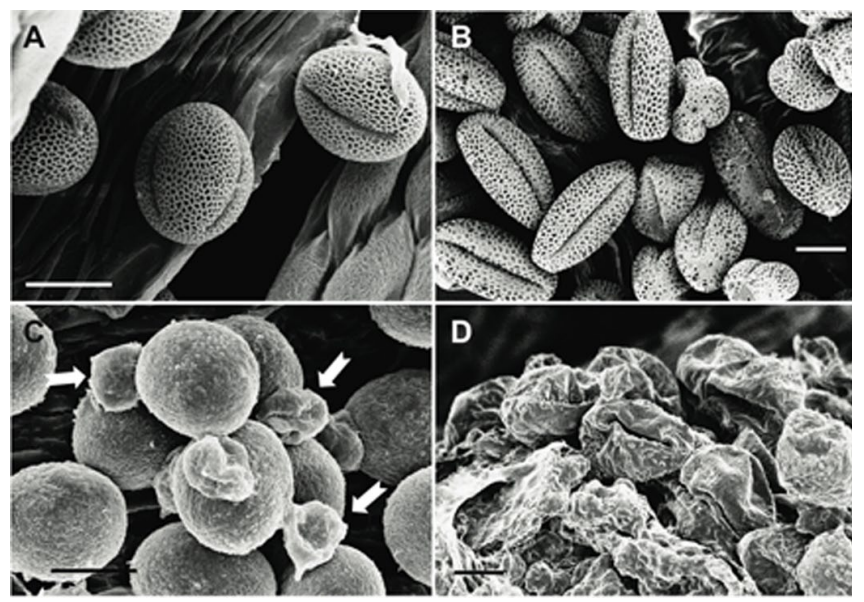

Figure 51. Comparison of pollen from CYP703A2 knockout plants and wild-type pollen.

(A) to (D) Surface structure of Arabidopsis pollen from wild-type Col-0 plants (A) and (B) and the CYP703A2 SLAT N56842 knockout line (C) and (D) after fixation of the tissues (A) and (C) or without fixation (B) and (D), monitored by scanning electron microscopy. Images modified from Morant et al. (2007). 
<smiles>COCCCCCCCC(=O)O</smiles>

Medium-chain saturated fatty acids

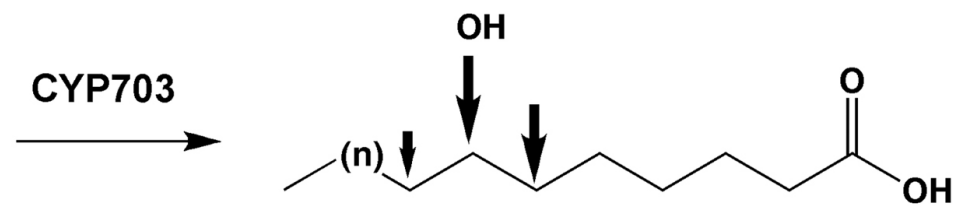

\section{In-chain hydroxylated fatty acid (precusors of sporopollenin?)}

Figure 52. The reactions catalyzed by yeast-expressed CYP703A2.

Yeast-expressed CYP703A2 catalyzes a single hydroxylation of medium chain fatty acids, mainly C12:0 and C10:0, in the chain with C7 as the main but not exclusive position of hydroxylation. Hydroxylated products obtained decrease with the distance from $\mathrm{C} 7$ as indicated by the size of the arrows on the top of the chain that roughly reflect reaction efficiency. A more specific reaction might occur in vivo.

matics. Dominguez et al. (1999), who developed one of the most sophisticated procedures for exine isolation, degradation and determination of its chemical composition, detected C6, C7 and C8 $\alpha, \omega$-dicarboxylic acids as the major degradation products that they describe as potentially deriving from ether-bound fatty acids. It thus seems plausible that CYP703A2 and its orthologs may provide essential hydroxy fatty acid precursors polymerized via ether bonds to form the backbone of the sporopollenin network.

Additional hypomorphic alleles caused by point mutations have subsequently been reported by Dobritsa et al. (2009). The mutants retained the reticulate pattern, but the exine was very thin and the pollen showed bare patches. The same authors described the impact of crosses with other exine deficient mutants (for more details see CYP704 section).

\section{CYP704}

There are three CYP704 genes in the genome of $A$. thaliana, two of them are clustered on chromosome 2 and belong to the CYP704A subfamily. The third is the only member of the CYP704B subfamily in Arabidopsis. The duplication that led to the CYP704A and CYP704B subfamilies predates the divergence between monocots and dicots (Nelson et al., 2004). Both subfamilies are thus expected to have important functions in Angiosperms. The function of CYP704As, which are mainly expressed in seeds, has not been reported. CYP704Bs share many common characteristics with CYP703s. Both of them appeared very early in the evolution of terrestrial plants. Three copies of each are found in the genome of the moss Physcomitrella patens, but they are usually represented as single copies in the genome of higher plants (Nelson, 2006). Their expression patterns are in addition very similar and indicate a role in spore or pollen development. Accordingly, CYP704B1, like CYP703A2 in Arabidopsis, is expressed in the anthers of the flower buds from stage 9 to stage 11 .

A large-scale genetic screen aimed at the identification of mutants with defects in pollen exine formation led to the function of CYP704B1 being elucidated (Dobritsa et al., 2009). These mutants were characterized by pollen that was not easily shed and appeared glossy under the microscope. When examined by laser confocal microscopy after staining with auramine $O$, the pollen lacked normal exine, replaced with stripes of material that
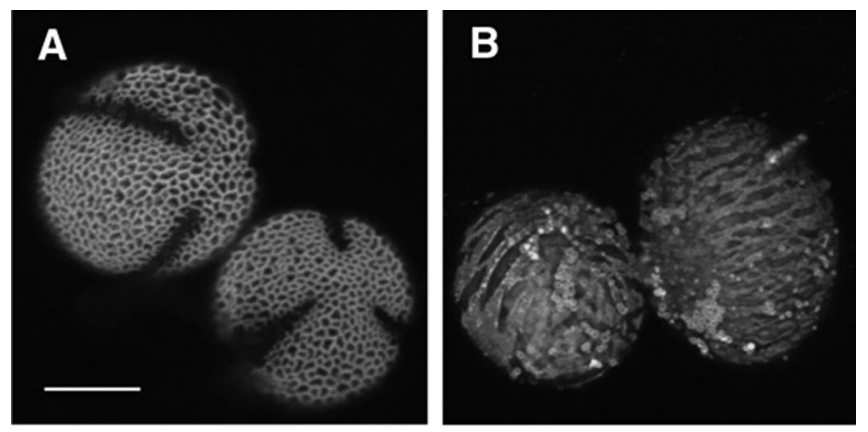

Figure 53. zebra mutants have severe defects in exine structure.

Scanning electron micrographs of the surface structure of pollen grains from the wild type (A) and the cyp704b1 mutant (allele SAlL_1149_B03; B). Samples were prepared without fixation. Reprinted from Dobritsa et al., (2009).

did not stain with auramine $\mathrm{O}$. This was described as a zebra pollen phenotype (Figure 53). Five mutant alleles were isolated, four resulting from point mutations, one from a T-DNA insertion. Examination of the latter by scanning electron microscopy revealed a complete lack of the reticulate exine pattern and pollen collapse under vacuum as previously reported for cyp703a2 mutants. Baculae, tectum and pollen coat were completely missing from the mutant pollen wall and replaced by a very thin layer of electron-dense material. Mutant pollen, in addition, became very sensitive to acetolysis. Despite such dramatic alterations in the pollen wall structure, no significant modification in plant fertility was observed when the mutants were grown under laboratory conditions.

CYP704s belong to the CYP86 clan of P450 enzymes and were thus predicted to be involved in fatty acid oxygenation (Nelson et al., 2004) for the formation of precursors of the sporopollenin biopolymer. In agreement with this prediction, CYP704B1 expressed in yeast was shown to hydroxylate saturated, unsaturated and epoxy C16 and C18 fatty acids in the $\omega$ position, both when they were readily present in the yeast microsomes and when added to the incubation medium. Lauric 


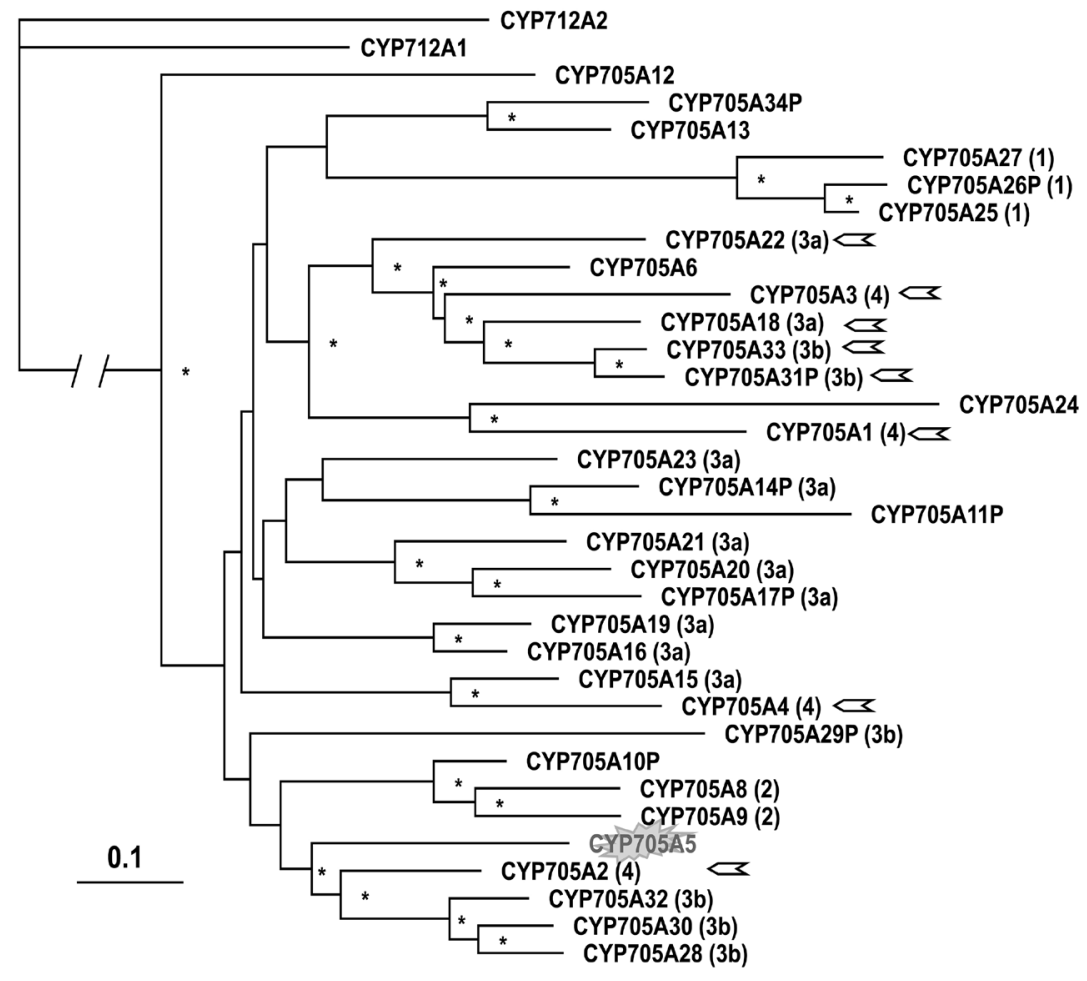

Figure 54. Phylogenetic tree of the Arabidopsis CYP705 family.

The remotely related subfamily CYP712 was used as outgroup and the broken line indicates greater distance than shown. Suffix P is used for pseudogenes. The number in brackets indicates location in one of five gene clusters: At1g50520 At1g50560 (1), At2g27000 At2g27010 (2), At3g20080 At3g20083 At3g20090 At3g20100 At3g20110 At3g20120 At3g20130 At3g20140 (3a), At3g20940 At3g20950 At3g20960 AT3G20935 (3b) and At4g15330 At4g15350 At4g15360 At4g15380 (4). The chevron highlights genes that were likely re-located after, or throughout duplication. The scale bar represents 0.1 amino acid changes. Highlighted the thalianol dehydrogenase. [Method: Maximum likelihood phylogeny using PhyML on manally curated alignment (Dialign2) of the deduced protein sequences (www.p450.kvl.dk), JTT Model of amino acids substitution, discrete gamma model with 4 rate categories (gamma shape parameter : 1.434), 100 bootstrap repetitions. Asterisks indicate bootstrap support of $70 \%$ and greater.]

acid, free and conjugated phenolic acids were not substrates of the enzyme.

The same zebra phenotype is observed for mutants of MS2, encoding a fatty acid reductase, and for mutants of CYP703A2. Double and triple mutants were thus generated by crossing cyp704b1 with ms2 and cyp703a2. None of these combinations of mutations had a stronger phenotype than the single mutant alleles in terms of reduction in the amount of viable pollen and in plant fertility compared to cyp704b1. CYP704B1, CYP703A2 and $M S 2$ are thus expected to participate in the biosynthesis of components of the same sporopollenin biopolymer.

\section{CYP705}

The Brassicaceae-specific CYP705 family with 33 genes is, after CYP71, the second largest in the Arabidopsis genome. The CYP705 family is a recent diversification of the CYP712 family. Their common ancestor is CYP93 (Nelson and Werck-Reichhart, 2011). Five clusters with CYP705 genes arranged adjacently on chromosomes 2, 3, 4 and 5 appear at first glance to be the result of tandem duplications and hence the primary source for the bloom of the CYP705 family. However, phylogenetic comparison shows that clusters on chromosome 3 both carry more distant relatives, e.g. CYP705A4 in cluster $3 a$ and CYP705A33 in cluster $3 b$ (Figure 54). Moreover, even though the four CYP705A1 to CYP705A4 are co-localised on chromosome 4 , their position in distinct clades indicates that local tandem-duplications are an unlikely source of the observed genetic diversity. This genomic distribution is more likely a sign of a substantial degree of de-novo re-organisation after initial duplication rounds. The presence of at least eight pseudogenes within the CYP705 family is another indicator of rapid evolution.

CYP705A5, together with CYP708A2, has been shown to form a contiguous operon-like gene cluster carrying as well the thalianol triterpene synthase and an acyltransferase (Figure 55) (Field and Osbourn, 2008). In root extracts of a T-DNA insertion mutant of CYP708A2, thalianol accumulated to higher levels and it was suggested that CYP708A2 is required for further conversion of the triterpenoid. Subsequently, increased amounts of thalian-diol, a product of a single hydroxylation reaction of thalianol, were detected in a null mutant of CYP705A5, while two peaks, identified as isomers of desaturated thalian-diol and present in wild type, were absent in the mutant. Based on these data, it was concluded that the contiguous genes encode consecutive 
A
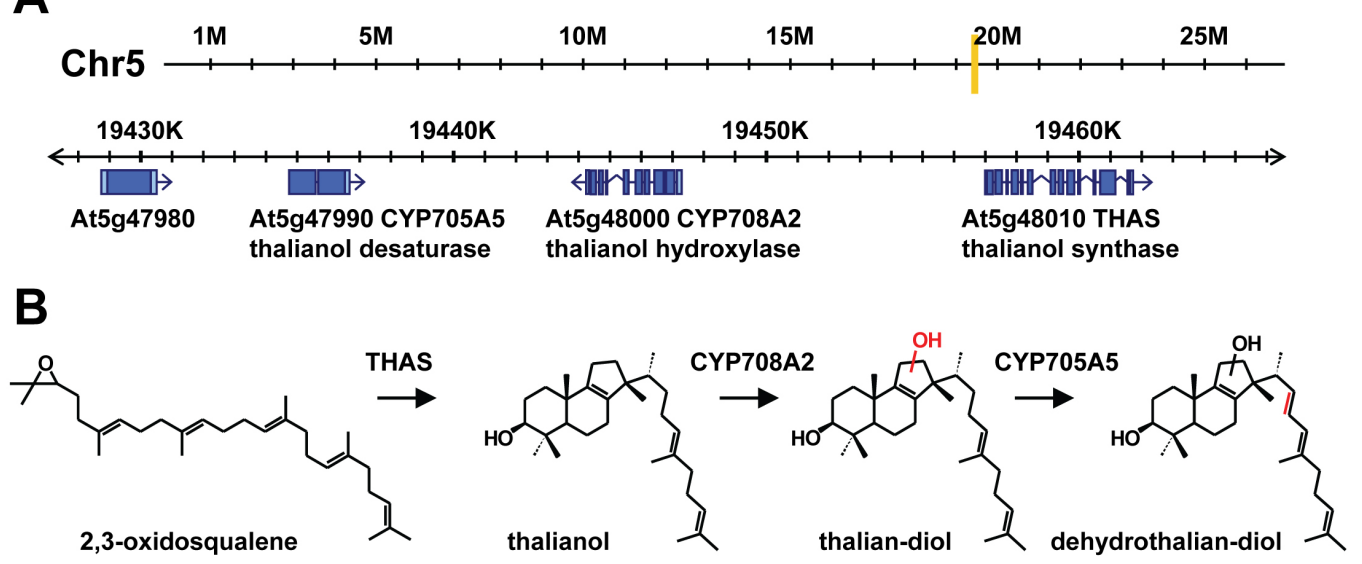

Figure 55. The thalianol biosynthetic gene cluster.

(A) The position of the cluster on chromosome 5 is given as yellow bar and the distribution of the genes encoding the thalianol cluster is shown in the second panel.

(B) Scheme of the pathway to modified thalianol derivatives. Position of the introduced hydroxyl function is unclear, and several compounds detected in Arabidopsis possibly represent positional isomers. Drawn after Field and Osbourn (2008).

enzymes in the biosynthesis of highly diversified thalianol derivatives via a triterpene synthase, hydroxylase and desaturase. The authors also showed that this gene cluster was duplicated in the Arabidopsis genome. This operon-like gene organization thus provides a prime example for the assembly of clusters of genes by gene duplication, neofunctionalisation, and genome reorganization extending beyond the CYP705 family.

\section{CYP706}

There are seven CYP706 genes in the Arabidopsis genome. Their function so far has not been reported. CYP706s belong to the CYP71 clan and appeared with early Angiosperms (in Magnoliids). Based on the function described for a CYP706 gene from cotton (Luo et al., 2001), they might be involved in terpenoid metabolism.

\section{CYP707}

As with many genes in hormone metabolism CYP707 appear early in plant evolution: the first CYP707-like genes are found in Lycopods. CYP707A genes encode abscisic acid (ABA) 8'-hydroxylases that inactivate ABA (Figure 56), and similar to most genes in terpenoid hormone metabolism, they belong to the CYP85 clan.

Four members of the CYP707A family, CYP707A1 to CYP707A4, exist in Arabidopsis, with each having distinctive roles due to tissueand temporal-specific expression patterns. Increased amounts of $\mathrm{ABA}$ are associated with the development of desiccation tolerance, maturation of seeds, seed dormancy and suppression of vivipary and not, as the name would suggest, in abscission. Rapid increase in $A B A$ levels is also observed in the water stress-induced closure of stomata. CYP707A1, CYP707A3 and CYP707A4 are expressed in seedlings whereas CYP707A2 exhibits weak expression (Umezawa et al., 2006). CYP707A2 is the major ABA hydroxylase

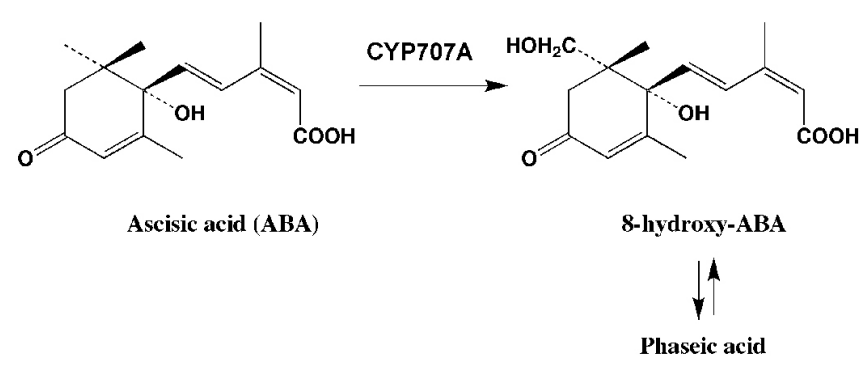

Figure 56. Hydroxylation of ABA catalyzed by the CYP707A enzymes. CYP707A catalyses the 8' hydroxylation of ABA.

involved in seed dormancy, whereas CYP707A3 has the predominant role in vegetative tissue (Kushiro et al., 2004; Okamoto et al., 2006; Umezawa et al., 2006). As expected, mutants defective in these P450s contain higher endogenous ABA levels, and exhibit enhanced stress resistance and increased seed dormancy (Kushiro et al., 2004; Saito et al., 2004). Constitutive over-expression of CYP707A genes result in reduced ABA content, providing compelling evidence that 8'-hydroxylation is the major route for ABA inactivation (Kushiro et al., 2004). CYP707As are induced by exogenous ABA and, when under water stress, by both dehydration and rehydration. They therefore play an essential role in maintaining optimal ABA levels (Kushiro et al., 2004; Saito et al., 2004).

Promoter GUS fusion transformed plants indicate that CYP707A1 expression is induced in guard cells in response to high humidity (Figure 57) and the cyp707a1 mutant is hypersensitive to exogenous ABA application in comparison to the cyp707a3 mutant (Okamoto et al., 2009). This indicates that CYP707A1 plays the major role in metabolizing $A B A$ in guard cells and therefore influencing 

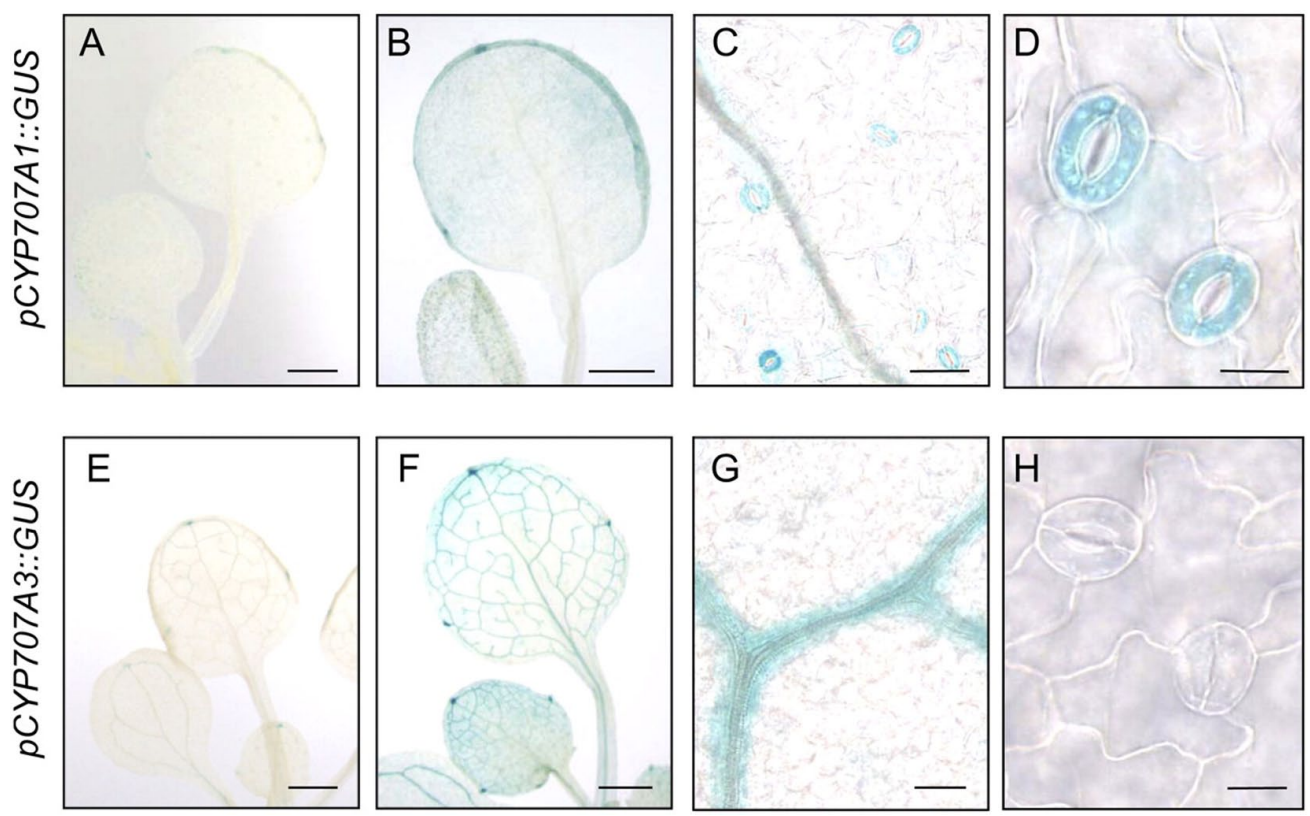

Figure 57. Spatial expression patterns of CYP707A1 and CYP707A3 in response to high humidity.

Expression of CYP707A1 promoter:GUS before (A) and after transferring to high humidity conditions (B-D). Expression of promoter CYP707A3:GUS before (E) and after transferring to high humidity conditions $(\mathrm{F}-\mathrm{H})$. Approximately 3-week-old plants were grown on soil in pots under $\mathrm{RH} 60 \%$ at $22^{\circ} \mathrm{C}$ condition. High humidity treatment (from RH $60 \%$ to $90 \%$ at $22^{\circ} \mathrm{C}$ ) was performed for $2 \mathrm{~h}$. Reprinted from Okamoto et al. (2009).

the stomatal aperture size. More detailed analysis of CYP707A3 has shown that this is the major CYP707A regulating ABA pools. It has higher expression than the other CYP707As and is induced during dehydration and rehydration processes. Mutant analyses show that disruption in CYP707A3 results in more drought tolerance, whereas over-expression results in an increased transpiration rate and reduced drought tolerance. CYP707A3 selectively binds (+)-ABA but not (-)-ABA, whereas the enzyme binds both enantiomers of $\mathrm{AHI} 1$ (a structural $\mathrm{ABA}$ analog) used as an $\mathrm{ABA}$ 8'-hydroxylase competitive inhibitor.

\section{CYP708}

The four members of this so far Brassicales-specific family belong to the CYP85 clan, which holds most of the characterised P450s involved in terpenoid metabolism. Together with CYP702s, CYP708s seem to be derived from an ancestral CYP87. Only the function of CYP708A2 has been described. This function is closely related to that of CYP705A5 in biosynthesis of the oxygenated triterpenoids derived from thalianol (Field and Osbourn, 2008). CYP708A2, CYP705A5, the thalianol triterpene synthase and an acyltransferase forms a contiguous operon-like gene cluster (Field and Osbourn, 2008). Two other clusters having the same gene pattern are found in the genome of Arabidopsis, which is indicative of the coevolution of associated genes in triterpenoid metabolism (Field and Osbourn, 2008). For more on the thalianol gene cluster, see CYP705.

\section{CYP709}

There are three CYP709 genes annotated in the genome of $A$. thaliana. CYP709s are present in ferns and belong to the CYP72 clan. Co-expression analyses (http://www-ibmp.u-strasbg.fr/ CY Pedia/) and fatty acid sub-terminal hydroxylase activity described in wheat (Kandel et al., 2005) suggests that at least some of them could be involved in seed lipid metabolism.

\section{CYP710}

The triterpenoid cycloartenol is the entry molecule in the primary metabolism for sterol-derived phytohormone biosynthesis, vital for a broad range of developmental processes and non-hormonal bulk sterol lipids that fulfill various cellular functions and modulate the physical properties of the cell membrane. In Arabidopsis, C-22 unsaturated stigmasterol and a mixture of brassicasterol and crinosterol dominate the phytosterols. CYP61 is the fungal P450 acting as sterol C-22 desaturase in the ergosterol pathway. Based on similarity, it was suggested that the CYP710 family carries the functional homologs of the fungal CYP61 enzymes.

CYP710s constitute a single family clan, conserved throughout all plants including green algae (Nelson et al., 2008). The family consists of four intronless genes, CYP710A1 to CYP710A4 in Arabidopsis. Heterologously expressed CYP710A1 and CYP710A2 were tested in vitro with the potential substrates $\beta$-sitosterol, campesterol, and 24-epi-campesterol (Morikawa et al., 2006). CYP710A1, and to a much lesser degree CYP710A2, 

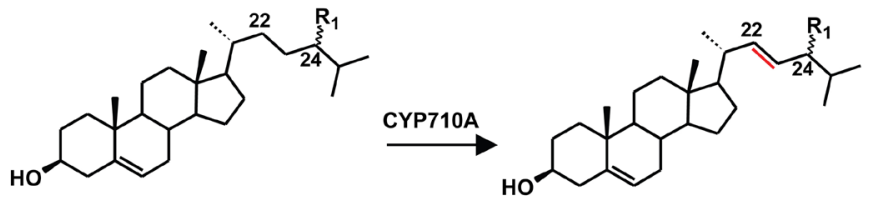

$\mathrm{R}_{1}$ 24(R) $\mathrm{CH}_{3}$ campesterol $\mathrm{R}_{1}$ 24(S) $\mathrm{CH}_{3}$ 24-epi-campesterol

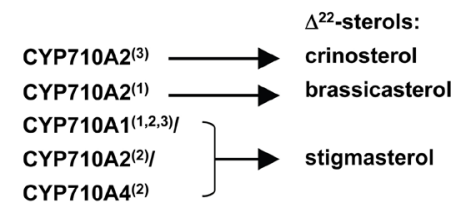

$R_{1}$ 24(R) $C_{2} H_{5} \quad \beta$-sitosterol

CYP710A4(2)
Figure 58. Common backbone structure of non-hormonal bulk sterol lipids and the desaturation reaction catalysed by Arabidopsis CYP710A.

Functional evidence through in vitro enzyme assay ${ }^{1}$ Morikawa et al., (2006); over-expression in planta ${ }^{2}$ Morikawa et al., (2006) and Arnqvist et al., (2008); null mutant allele ${ }^{3}$ Morikawa et al., (2006) and Griebel and Zeier, (2010).

catalysed the C-22 desaturation converting $\beta$-sitosterol to stigmasterol (Figure 58). 4-epi-campesterol, but not the 24R-epimer campesterol, was converted by CYP710A2 to brassicasterol, albeit with low activity (Morikawa et al., 2006). CYP710A1, CY$P 710 A 2$ and CYP710A4 were overexpressed in transgenic Arabidopsis. CYP710A1 and CYP710A4 over-expression resulted in an increase of stigmasterol, with no significant changes in accumulation of brassicasterol/crinosterol (Morikawa et al., 2006; Arnqvist et al., 2008), while over-expression of CYP710A2 resulted in increased levels of brassicasterol/crinosterol (Morikawa et al., 2006). In a cyp710a2 null mutant line, formation of brassicasterol/crinosterol was not detectable, corroborating the function of CYP710A2 in biosynthesis of the C-24 methyl-sterols (Morikawa et al., 2006) (Figure 58). Griebel and Zeier (2010) demonstrated a function of the conversion of $\beta$-sitosterol to stigmasterol in the interaction of Arabidopsis and bacterial pathogens. It was shown that stigmasterol accumulates after infection with Pseudomonas syringae. Furthermore, cyp710a1 mutant lines with reduced C22desaturase activity and resulting reduced stigmasterol accumulation displayed an increased resistance compared to wild-type plants. Exogenous supplementation of stigmasterol was sufficient to compensate this phenotype (Griebel and Zeier, 2010).

\section{CYP711}

The CYP711 family constitutes its own clan due to insufficient similarity with other families. It is found ubiquitously in land plants from Lycopods. The single copy founding member of the CYP711 family CYP711A1 was identified in Arabidopsis through map based cloning of the locus of the max1 mutant (more axillary growth) which displayed increased shoot branching (Booker at al., 2005). Based on the findings that application of the synthetic strigolactone analogue GR24 to rosette axillary buds of $\max 1$ plants and to a strigolactone deficient pea mutant upstream in the pathway of CYP711A suppressed max1-dependent bud outgrowth, it was concluded that CYP711A1 acts on a mobile signal intermediate of strigolactones, novel shoot multiplication regulators (Gomez-Roldan et al., 2008).
Strigolactones were also described to play a dual role in the rhizospere as signals used by arbuscular mycorrhizal (AM) fungi and root parasitic plants for host plant detection (Akiyama et al., 2005 and Bouwmeester et al., 2003). The induction of seed germination of parasitic plants was significantly reduced in response to root exudates of max1, paralleled by a strong reduction of detectable strigolactones in max1 (Kohlen et al., 2011).

Strigolactones share a unique structure consisting of a tricyclic lactone coupled via an ether bond to 3-methylfuran-2(5H)-one. They represent a class of terpenoids (apocarotenoids) derived from oxidative cleavage of $\mathrm{C}-40$ carotenoids and more than ten structural variants have been detected in plants. A distinct pattern of hydroxylation, further oxidation to ketones and epoxidation of the original backbone structure (Koichi et al., 2009) indicates action of P450s beyond CYP711A (Figure 59).

Expression of Arabidopsis CYP711A1 was shown to be strongly circadian regulated under three of four different conditions tested and trailed behind the phasing expression of carotenoid anabolic P450s CYP97A3, CYP97C1, and that of CYP97B3 between two and seven hours, depending on the condition tested (Pan et al., 2009), supporting the hypothesized biosynthetic route.

So far, neither the substrate nor putative products of the P450s of the CYP711 family have been identified. Major challenges in the unequivocal solution of the biosynthetic pathway leading to the structurally diverse strigolactones are the low concentration of inherently unstable metabolites and potential functional redundancies within the gene families involved.

\section{CYP712}

The function of the two CYP712s in the Arabidopsis genome has not so far been reported. They belong to the CYP71 clan. CYP712 orthologs are not reported before core Eudicots. They seemingly evolved from an ancestral CYP93. It was suggested that CYP705 and CYP712 families should be merged to reflect the close evolutionary relationship that became clear after the sequencing of several plant genomes (Nelson et al., 2008). CYP712s might thus be functionally related to CYP93/CYP705 as also suggested by physical clustering in several plant genomes.

\section{CYP713}

The members of the CYP713 family have been renamed: CYP713A2 became CYP71A28 and the CYP713A1 pseudogene is now CYP71A27P.

\section{CYP714}

The CYP714 family is found in the CYP72 clan, which includes members involved in brassinosteroid catabolism. CYP714s appeared with flowering plants, the first being detected in the basal angiosperms magnoliids. The two CYP714As annotated in the Arabidopsis genome are tandemly duplicated on chromosome 4 and they encode homologs of the rice elongated uppermost internode (EUI1), which encodes CYP714D1 and has been shown to inactivate the non-13-hydroxylated gibberellins such as $\mathrm{GA}_{4}$ by 16, 17-epoxidation (Ma et al., 2006; Zhu et al, 2006). The func- 


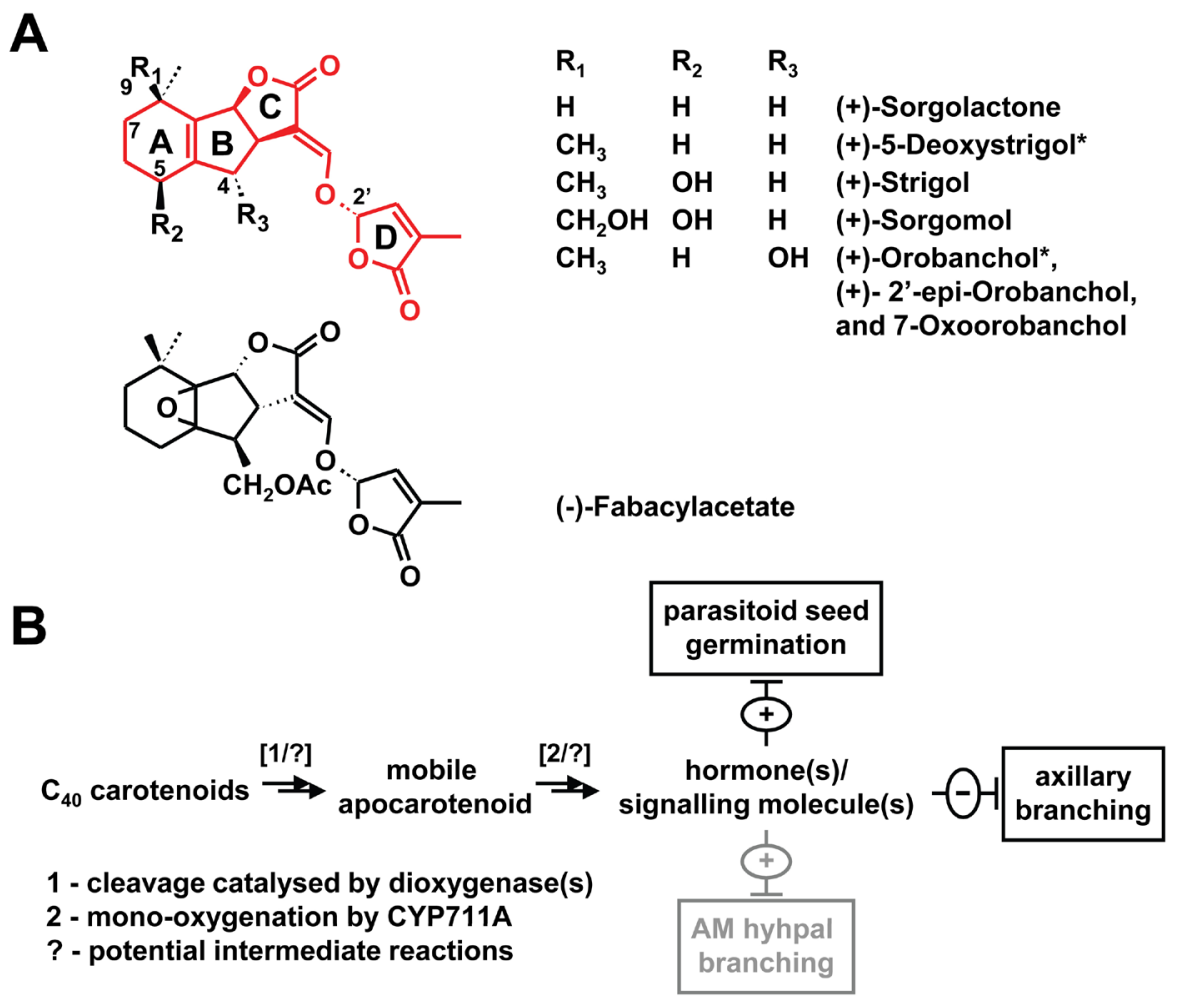

Figure 59. The possible role(s) of CYP711 in the strigolacone pathway.

(A) Common backbone structure (in red) and various oxidative decorations leading to naturally occurring strigolactones.

(B) Hypothesized biosynthetic pathway leading to strigolactones based hormones and signaling molecules. Asterisks indicate strigolactones detected in Arabidopsis. Shown in grey are functions not found in Arabidopsis.

tion of the Arabidopsis members remained unclear for a long time, with the only indication being a Japanese patent describing a function of CYP714A2 in the production of steviol glycosides, natural sweeteners and hydroxy-derivatives of the diterpenoid kaurenoic acid (Yamaguchi, 2008). However, the most recent study of Zhang and co-workers (2011) is shedding light on the in planta function of Arabidopsis CYP714A1 and CYP714A2. Expression analysis, combination of single null mutants with RNAi for the corresponding second homologue, and transgenic lines constitutively overexpressing individual P450s were used together with profiling of GAs to demonstrate deactivation of GAs through functionally redundant CYP714A1 and CYP714A2. The genes showed partially overlapping expression pattern, indicating some degree of subfunctionalisation, yet single null mutants did not reveal a phenotype. In double mutants using RNAi to target the second homologue, an increased biomass accumulation was described, possibly due to increased cell expansion. In contrast, overexpression of CYP714A1 and CYP714A2 driven by the constitutive $35 \mathrm{~S}$ promoter resulted in stunted growth, which could be restored by exogenous application of GAs. This was paralleled by complex changes in the accumulation of numerous GAs, which led the authors to conclude that CYP714A1 and CYP714A2 function in the inactivation of early GA intermediates.

\section{CYP715}

A single CYP715 gene is found in the Arabidopsis, rice and most other plant genomes. It belongs to the CYP72 clan and appeared before the divergence between Gymnosperms and Angiosperms. In spite of this low copy, indicative of an important function, the latter is not yet reported.

\section{CYP716}

There are two CYP716 genes clustered on chromosome 5 in Arabidopsis. Their function is not reported to date. CYP716s belong to the CYP85 clan and evolved early with terrestrial plants. They are present in the moss genome, but not in monocots (Nelson and Werck-Reichhart, 2011). Co-expression analysis associated them with triterpene metabolism (Ehlting et al., 2008).

\section{CYP717}

The CYP717 family is not represented in A. thaliana. A single CYP717 gene was registered in daylily, ultimately renamed CYP81J1. 


\section{CYP718}

A single CYP718 gene is present in the genome of Arabidopsis. It seems to result from a duplication of an ancestral CYP716 that occurred in early dicot evolution. Its function is not described. As only very few ESTs are reported for CYP718s in all plants, they have been suggested to be expressed at very low levels or very transiently (Nelson et al., 2008).

\section{CYP719}

The CYP719 family is not represented in A. thaliana. CYP719s are reported to contribute to the biosynthesis of alkaloids in Ranunculales and Aristolochiales (see e.g. Ikezawa et al., 2003; Gesell et al., 2009).

\section{CYP720}

The CYP720 family falls into the CYP85 clan with members involved in terpenoid metabolism. A single copy CYP720 gene is present in Arabidopsis and in other sequenced dicot genomes. It appears to have evolved from an ancestral CYP90 gene in higher plants (Nelson et al., 2008). The function of CYP720A members is currently unknown and the expression levels are generally low, based on EST evidence across all species with representatives. The next closest homologues are found in the conifer specific CYP720B subfamily which underwent a subfamily specific bloom in spruce and pine and is involved in resin diterpene oxygenation (Hamberger and Bohlmann, 2006).

\section{CYP721}

The single CYP721 gene in Arabidopsis is expressed in most plant tissues but its function has not been reported. It belongs to the CYP72 clan and evolved with lower Angiosperms (it is first found in Amborella).

\section{CYP722}

The CYP722 family is represented by a single gene in $A$. thaliana, rice and most other plant genomes (Nelson and Werck-Reichhart, 2011). It belongs to the CYP85 clan and evolved in Angiosperms. It is found in monocots and dicots, but not in Magnoliids (Nelson et al., 2008). Its function has not been reported.

\section{CYP723}

The CYP723 family is present only in Monocots.

\section{CYP724}

In Arabidopsis the CYP724 family has only one member and is evolutionally closely related to P450s involved in brassinosteroids synthesis, in particular CYP90Bs. As yet the role of CYP724 in Arabidopsis has not been shown, however, in tomato and rice this family is involved in the $\mathrm{C}-22$ hydroxylation of BRs (Figure 38). The rice dwarf11 (d11) mutant has a de- fect in CYP724B1 and exhibits a weak dwarf phenotype having reduced seed length similar to the rice cyp90d2 (d2) mutant (Tanabe et al., 2005). The $d 11$ and osdwarf4/cyp90b2 double mutant has a severe dwarf phenotype and in vitro analysis of enzymatic activity confirms that both CYP724B1 and CYP90B2 from rice have C-22 hydroxylase activity (Sakamoto et al., 2006). Interestingly, the weak osdwarf4 mutant phenotype generates plants with enhanced biomass and yield, which indicates a novel method for crop improvement (Sakamoto et al., 2006). The tomato homolog, CYP724B2, has also been shown to have C-22 hydroxylase activity using a baculovirus-produced enzyme (Ohnishi et al., 2006c).

Since the known C-22 hydroxylase, CYP90B1, in Arabidopsis has such a strong phenotype when mutated it would suggest that CYP724A1 lacks sufficient expression to provide functional redundancy.

\section{CYP725 to CYP733}

These families are not represented in A. thaliana. They are either taxa-specific families or families that have been lost in crucifers or earlier during evolution (e.g. CYP727, CYP728, CYP729, and CYP733).

\section{CYP734}

Arabidopsis has one member of the CYP734 family. CYP734A1 belongs to the CYP72 clan and was initially classified as $C Y$ P72B1. CYP734A1 has been shown to catalyse the C-26 hydroxylation that inactivates brassinosteroids (Figure 38). An activation tagged mutant of CYP734A1 was identified in a genetic screen for suppressors of the phytochrome $B$ mutant phenotype that is defective in photomorphogenesis. The BAS1 (phyB activation-tagged suppressor1) mutant that was identified resulted in a dwarfed plant that responded to brassinosteroid (BR) application (Neff et al., 1999). Functional analysis has shown that CYP734A1 catalyses C-26 hydroxylation of the most bioactive brassinosteroids including both castasterone and brassinolide (Turk et al., 2003).

CYP734A1 has been shown to be BR inducible (Tanaka et al., 2005) and this requires signaling through the BR receptor (Choe et al., 2001). Regulation of BR levels by CYP734As is common to many species as homologs have been recovered, e.g. in tomato, that have been shown to encode BR C-26 hydroxylases (Ohnishi et al., 2006b). This indicates that, unlike CYP72C, which seems to be Arabidopsis specific, the CYP734As catalyze a universal step in inactivating BRs.

\section{CYP735}

CYP735 is an ancient CYP family that appeared with ferns (Nelson and Werck-Reichhart, 2011). Arabidopsis has two CYP735 family members, CYP735A1 and CYP735A2. They were formerly included in the CYP709 family and belong to the CYP72 clan. CYP735A1 and CYP735A2 hydroxylate cytokinins on the isoprene side chain (Figure 60). Cytokinins are made from 5'AMP with either an aromatic or an isoprene unit attached to N6 and 


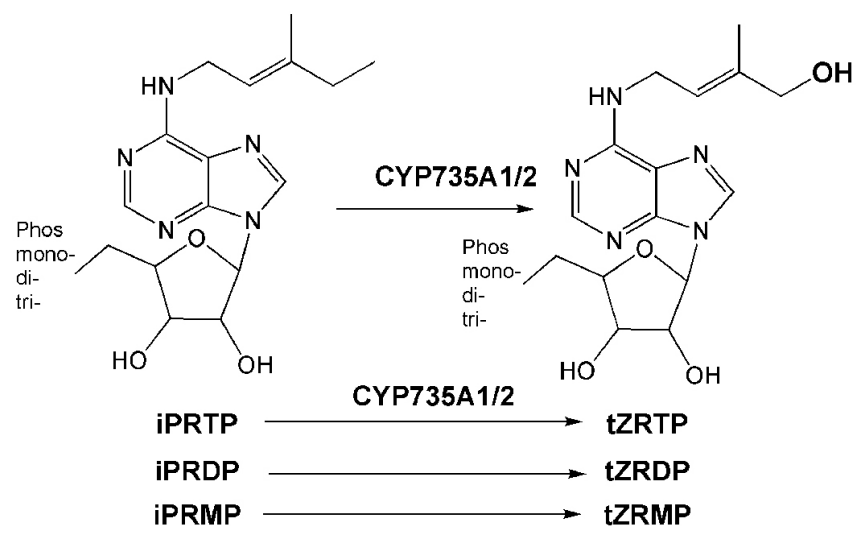

Figure 60. CYP735A members catalyze hydroxylation of cytokinins.

CYP735A1 and CYP735A2 can hydroxylate the side chain of cytokinins in the synthesis of trans-zeatin. They can convert the following mono-, di- and tri- phosphates forms into their respective hydroxylated products; iPRMP, isopentenyladenine riboside 5'-monophosphate to tZRMP, transzeatin riboside 5'-monophosphate; iPRDP, isopentenyladenine riboside 5'-diphosphate to tZRDP, trans-zeatin riboside 5'-diphosphate; iPRTP, isopentenyladenine riboside 5'-triphosphate to tZRTP, trans-zeatin riboside 5 '-triphosphate.

modified in various ways. They are activators of cell proliferation and are involved in numerous plant growth and development processes. This includes the regulation of plant reproduction, leaf senescence and apical dominance. Cytokinin signaling interacts with other hormone signaling pathways, for example, a high cytokinin to auxin ratio will produce shoots in callus tissue, whereas high auxin and low cytokinin will initiate root formation.

CYP735As were identified in a screen where co-expression of Arabidopsis IPT4 (adenosine phosphate-isopentenyltranferase 4) and the P450, was carried out in yeast that also expressed the Arabidopsis NADPH-P450 reductase (Takei et al., 2004). CYP735A1 and CYP735A2 have different patterns of expression, with CYP735A2 being more highly expressed in roots and also induced by exogenous application of cytokinins (Takei et al., 2004).

\section{FAMILIES BEYOND CYP735 ARE NOT REPRESENTED IN ARABIDOPSIS}

\section{Outlook and Future Research}

In the last ten years, there has been a huge acceleration in plant P450 gene discovery and in our understanding of their diversity and function. Despite this acceleration, the function of more than $70 \%$ of the P450s found in the Arabidopsis genome is still unknown or poorly understood. When information is available on their biochemical function, understanding of the role of these P450s in the plant is often missing. In contrast, several null mutants have been described which show interesting phenotypes, but no explanation for these phenotypes has been provided. A large field of investigation is thus opened that should lead to the discovery of novel pathways and reveal so far overlooked plant metabolites with hormonal or defense signaling activity.

As more information becomes available on P450 functions, via expression, phylogeny, evolution and (co)-localization in the plant genome(s), new leads can be found to accelerate the discovery of ophan gene function(s). Phylogeny was one of the best guides in the first stage of gene discovery (see e.g. Benveniste et al., 1998). It can now be combined with comparative CYPomes analysis to spot genes of interest in the metabolism of hormones or defense compounds (Nelson and Werck-Reichhart, 2011). Another powerful approach is gene co-expression analysis. For example, P450 co-expression with terpene synthases provides many candidates for the elucidation of the different branches of the terpenoid pathway that is still very superficially explored in Arabidopsis (Ehlting et al., 2008). More valuable leads are provided by gene clustering in the plant genome(s). Five independent biosynthetic gene clusters involving P450s were reported in plants, four of which are involved in terpenoid metabolism (Osbourn and Field, 2009). They often consist of co-regulated genes with distinct functions that constitute segments of thus far exclusively plant defense metabolic routes. Biosynthetic gene clusters catalyse production of various diterpenoid phytoalexins of the momilactone and phytocassane class in rice, of benzoxazinones in corn, and of the triterpenoids avenacin and thalianol in oat and Arabidopsis, respectively (Frey et al., 1997; Qi et al., 2004; Field and Osbourn, 2008; Shimura et al., 2007). Several Arabidopsis genes from P450 families with members involved in terpenoid metabolism reside in close vicinity to terpene synthase or other metabolic genes. These are usually co-regulated. For example, two sesquiterpene [Z]- $\gamma$-bisabolene synthases are localized closely together, each paired head-to-head with a P450 gene of the CYP71A subfamily. A strong expression of CYP71A19 in roots and transcript accumulation in response to wounding and other forms of stress in roots or leaves is shared with its clustered terpene synthase gene AtTPS12 (Ro et al., 2006).

Despite progress in the prediction of putative CYP functions, challenges remain for the functional characterisation of CYPs with limited knowledge about the putative classes of products and the notorious lack of available substrate libraries. In the case of $\mathrm{P} 450$ s hypothesised to oxygenate structurally and stereochemically complex terpene backbones, the latter obstacle may be overcome by providing the P450 putative substrates through coupled assays with heterologously expressed terpene synthases. When investigations have to be carried out with mutant plants, the problem is further complicated by the very local accumulation, low concentration and lability of some reaction products that are directly channeled into metabolic pathways, and by the fact that the latter can be shut down when the flux is interrupted. For identification of substrate and reaction products, the extraction and analytical procedures have to be adapted for each type of metabolite when those can be predicted. Integrated "omics" approaches, in particular the combination of global transcriptomics with the emerging field of non-targeted metabolomics appear promising for the discovery of novel pathway intermediates and low abundant metabolites. For example, Fourier transform ion cyclotron resonance mass (FTICR-MS) based metabolomics, recently developed for the analysis of ultra-high resolution broadband measurements of complex metabolite mixtures, have allowed predic- 
tion of enzyme reactions reflected in specific differences in $\mathrm{m} / \mathrm{z}$ values, e.g. the addition of $+\mathrm{O}(\Delta \mathrm{m} / \mathrm{z}=15.99491)$ as the product of a mono-oxygenase (Ohta et al., 2010). This approach can be powerful for the reconstruction of metabolic pathways via analysis of metabolic links in the KNApSAcK database. The KNApSAcK database currently holds 50048 metabolites and 101500 metabolite-species pair entries as of April 5, 2011 (http://kanaya. naist.jp/KNApSAcK/). It may, however, not be applicable for elusive metabolites present in low amounts or very specific tissues.

\section{AKNOWLEDGMENTS}

D.W. and R.H. are grateful to the European Comission for the funding of the FP7 SmartCell project. D.W. in addition acknowledges the Agence Nationale de la Recherche for the funding of the METAMAP and PHENOWALL projects. B.H. acknowledges UNIK Synthetic Biology, funded by the Danish Ministry of Science, Technology and Innovation. FB is grateful to the European Commission for the funding of the FP7-People Marie Curie International Reintegration Grant \#224941 BIOLIPOL. G.B. has been funded by the European Commission and Biotechnology and Biological Sciences Research Council.

\section{REFERENCES}

Abdulrazzak, N., Pollet, B., Ehlting, J., Larsen, K., Asnaghi, C., Ronseau, S., Proux, C., Erhardt, M., Seltzer, V., Renou, J.P., Ullmann, P., Pauly, M., Lapierre, C., and Werck-Reichhart, D. (2006). A coumaroyl-ester-3-hydroxylase insertion mutant reveals the existence of nonredundant meta-hydroxylation pathways and essential roles for phenolic precursors in cell expansion and plant growth. Plant Physiol. 140: $30-48$

Abrahams, S., Tanner, G.J., Larkin, P.J., and Ashton, A.R. (2002). Identification and biochemical characterization of mutants in the proanthocyanidin pathway in Arabidopsis. Plant Physiol. 130: 561-76.

Adamski, N.M., Anastasiou, E., Eriksson, S., O'Neill, C.M., and Lenhard, M. (2009). Local maternal control of seed size by KLUH/CYP78A5-dependent growth signaling. Proc. Natl. Acad. Sci. USA 106: 20115-20120.

Akiyama, K., Matsuzaki, K.I., and Hayashi, H. (2005). Plant sesquiterpenes induce hyphal branching in arbuscular mycorrhizal fungi. Nature 435: 824-827.

Anastasiou, E., Kenz, S., Gerstung, M., MacLean, D., Timmer, J., Fleck, C., and Lenhard, M. (2007) Control of plant organ size by KLUH/ CYP78A5-dependent intercellular signaling. Dev Cell. 13: 843-56.

Arnqvist, L., Persson, M., Jonsson, L., Dutta, P.C., and Sitbon, F. (2008). Over-expression of CYP710A1 and CYP710A4 in transgenic Arabidopsis plants increases the level of stigmasterol at the expense of sitosterol. Planta 227: 309-317.

Asami, T., Mizutani, M., Fujioka, S., Goda, H., Min, Y.K., Shimada, Y., Nakano, T., Takatsuto, S., Matsuyama, T., Nagata, N., Sakata, K., and Yoshida, S. (2001). Selective interaction of triazole derivatives with DWF4, a cytochrome P450 monooxygenase of the brassinosteroid biosynthetic pathway, correlates with brassinosteroid deficiency in planta. J. Biol. Chem. 276: 25687-25691.

Asami, T., Mizutani, M., Shimada, Y., Goda, H., Kitahata, N., Sekimata, K., Han, S.-Y., Fujioka, S., Takatsuto, S., Sakata, K., and Yoshida, S. (2003). Triadimefon, a fungicidal triazole-type P450 inhibitor, induces brassinosteroid deficiency-like phenotypes in plants and binds to DWF4 protein in the brassinosteroid biosynthesis pathway.
Biochem. J. 369: 71-76

Ayabe, S.I., and Akashi, T. (2006). Cytochrome P450s in flavonoid metabolism. Phytochemistry Rev. 5: 271-282.

Azpiroz, R., Wu, Y., LoCascio, J. C., and Feldmann, K. A. (1998). An Arabidopsis brassinosteroid-dependent mutant is blocked in cell elongation. Plant Cell 10: 219-230.

Bak, S., and Feyereisen, R. (2001). The involvement of two P450 enzymes, CYP83B1 and CYP83A1, in auxin homeostasis and glucosinolate biosynthesis. Plant Physiol. 127: 108-118.

Bak, S., Kahn, R. A., Olsen, C. E., and Halkier, B. A. (1997). Cloning and expression in Escherichia coli of the obtusifoliol 14 alpha-demethylase of Sorghum bicolor (L.) Moench, a cytochrome P450 orthologous to the sterol 14 alpha-demethylases (CYP51) from fungi and mammals. Plant J. 11: 191-201.

Bak, S., Tax, F. E., Feldmann, K. A., Galbraith, D. W., and Feyereisen, R. (2001). CYP83B1, a Cytochrome P450 at the metabolic branch point in auxin and indole glucosinolate biosynthesis in Arabidopsis. Plant Cell 13: 101-111.

Bak, S., Paquette, S.M., Morant, M., Rasmussen, A.V., Saito, S., Bjarnholt, N., Zagrobelny, M., Jørgensen, K., Hamann, T., Osmani, S., Simonsen, H.T., Pérez, R.S., van Hesswijck, T.B., Jørgensen, B., and Møller, B.L. (2006). Cyanogenic glycosides: a case study for evolution and application of cytochromes P450. Phytochemistry Rev. 5: 309-329.

Bancos, S., Nomura, T., Sato, T., Molnár, G., Bishop, G.J., Koncz, C., Yokota, T., Nagy, F., and Szekeres, M. (2002). Regulation of transcript levels of the Arabidopsis cytochrome P450 genes involved in brassinosteroid biosynthesis. Plant Physiol. 130: 504-513.

Bancos, S., Szatmari, A.M., Castle, J., Kozma-Bognar, L., Shibata, K., Yokota, T., Bishop, G.J., Nagy, F., and Szekeres, M. (2006). Diurnal regulation of the brassinosteroid-biosynthetic CPD gene in Arabidopsis. Plant Physiol. 141: 299-309.

Barlier, I., Kowalczyk, M., Marchant, A., Ljung, K., Bhalerao, R., Bennett, M., Sandberg, G., and Bellini, C. (2000). The SUR2 gene of Arabidopsis thaliana encodes the cytochrome P450 CYP83B1, a modulator of auxin homeostasis. Proc. Natl. Acad. Sci. USA 97: 14819-14824.

Bate, N.J., Sivasankar, S., Moxon, C., Riley, J.M., Thompson, J.E., and Rothstein, S.J. (1998). Molecular characterization of an Arabidopsis gene encoding hydroperoxide lyase, a cytochrome P-450 that is wound inducible. Plant Physiol. 117: 1393-400.

Bednarek, P., Pislewska-Bednarek, M., Svatos, A., Schneider, B., Doubsky, J., Mansurova, M., Humphry, M., Consonni, C., Panstruga, R., Sanchez-Vallet, A., Molina, A., and Schulze-Lefert, P. (2009). A glucosinolate metabolism pathway in living plant cells mediates broad-spectrum antifungal defense. Science 323: 101-106.

Beeckman, T., DeRycke, R., Viane, R., and Inze, D. (2000) Histological study of seed coat development in Arabidopsis thaliana. J. Plant Res. 113: $139-148$

Bell-Lelong, D.A., Cusumano, J.C., Meyer, K., and Chapple, C. (1997). Cinnamate-4-hydroxylase expression in Arabidopsis. Regulation in response to development and the environment. Plant Physiol. 113: 729738.

Benveniste, I., Tijet, N., Adas, F., Philipps, G., Salaün, J.P., and Durst, F. (1998). CYP86A1 from Arabidopsis thaliana encodes a cytochrome P450-dependent fatty acid omega-hydroxylase. Biochem. Biophys. Res. Commun. 243: 688-693.

Benveniste, I., Bronner, R., Wang, Y., Compagnon, V., Michler, P., Schreiber, L., Salaün, J.P., Durst, F., and Pinot, F. (2005). CYP94A1, a plant cytochrome $\mathrm{P} 450$-catalyzing fatty acid $\omega$-hydroxylase, is selectively induces by chemical stress in Vicia sativa seedlings. Planta 221: 881-890.

Bernhardt, R. (2006). Cytochromes P450 as versatile biocatalysts. J. Bio- 
technol. 124: 128-45.

Bishop, G.J., Harrison, K., and Jones, J.D. (1996). The tomato Dwarf gene isolated by heterologous transposon tagging encodes the first member of a new cytochrome P450 family. Plant Cell 8: 959-969.

Bishop, G.J., Nomura, T., Yokota, T., Harrison, K., Noguchi, T., Fujioka, S., Takatsuto, S., Jones, J.D., and Kamiya, Y. (1999). The tomato DWARF enzyme catalyses C-6 oxidation in brassinosteroid biosynthesis. Proc. Natl. Acad. Sci. USA 96: 1761-1766.

Bishop, G.J. (2007). Refining the plant steroid hormone biosynthesis pathway. Trends Plant Sci. 12: 377-380.

Boavida, L.C., Shuai, B., Yu, H.J., Pagnussat, G.C., Sundaresan, V., and McCormick S. (2009). A collection of Ds insertional mutants associated with defects in male gametophyte development and function in Arabidopsis thaliana. Genetics 181:1369-85.

Boland, W., Gäbler, A., Gilbert, M., and Feng, Z. (1998). Biosynthesis of C11 and C16 homoterpenes in higher plants; stereochemistry of the C-C-bond cleavage reaction. Tetrahedron 54: 14725-14736.

Booker, J., Sieberer, T., Wright, W., Williamson, L., Willett, B., Stirnberg, P., Turnbull, C., Srinivasan, M., Goddard, P., and Leyser, O. (2005). MAX1 encodes a cytochrome P450 family member that acts downstream of MAX3/4 to produce a carotenoid-derived branch-inhibiting hormone. Dev. Cell. 8: 443-449.

Bouwmeester, H.J., Matusova, R., Zhongkui, S., and Beale, M.H. (2003). Secondary metabolite signalling in host-parasitic plant interactions. Curr. Opin. Plant Biol. 6: 358-364.

Bozak, K.R., Yu, H., Sirevag, R., and Christoffersen, R.E. (1990). Sequence analysis of ripening-related cytochrome P-450 cDNAs from avocado fruit. Proc. Natl. Acad. Sci. USA 87: 3904-3908.

Brader, G., Mikkelsen, M.D., Halkier, B.A., and Tapio Palva, E. (2006). Altering glucosinolate profiles modulates disease resistance in plants. Plant J. 46: 758-67.

Bruce, T.J.A., Matthes, M.C., Chamberlain, K., Woodcock, C.M., Mohib, A., Webster, B., Smart, L.E., Birkett, M.A., Pickett, J.A., and Napier, J.A. (2008). cis-Jasmone induces Arabidopsis genes that affect the chemical ecology of multitrophic interactions with aphids and their parasitoids. Proc. Natl. Acad. Sci. USA 105: 4553-4558.

Böttcher, C., Westphal, L., Schmotz, C., Prade, E., Scheel, D., and Glawischnig, E. (2009). The multifunctional enzyme CYP71B15 (PHYTOALEXIN DEFICIENT3) converts cysteine-indole-3-acetonitrile to camalexin in the indole-3-acetonitrile metabolic network of Arabidopsis thaliana. Plant Cell 21: 1830-1845.

Cabello-Hurtado, F., Zimmerlin, A., Rahier, A., Taton, M., DeRose, R., Nedelkina, S., Batard, Y., Durst, F., Pallett, K. E., and Werck-Reichhart, D. (1997). Cloning and functional expression in yeast of a cDNA coding for an obtusifoliol 14a-demethylase (CYP51) in wheat. Biophys. Biochem. Res. Commun. 230: 381-385.

Chaban, C., Waller, F., Furuya, M., and Nick, P. (2003). Auxin responsiveness of a novel cytochrome p450 in rice coleoptiles. Plant Physiol. 133: 2000-2009.

Chapple, C.C.S., Vogt, T., Ellis, B. E., and Somerville, C.R. (1992). An Arabidopsis mutant defective in the general phenylpropanoid pathway. Plant Cell 4: 1413-1424.

Castle, J., Szekeres, M., Jenkins, G., and Bishop, G.J. (2005). Unique and overlapping expression patterns of Arabidopsis CYP85 genes involved in brassinosteroid C-6 oxidation. Plant Mol. Biol. 57: 129-140.

Chen, H., Jiang, H., and Morgan, J.A. (2007). Non-natural cinnamic acid derivatives as substrates of cinnamate 4-hydroxylase. Phytochemistry 68: 306-311.

Choe, S., Dilkes, B.P., Fujioka, S., Takatsuto, S., Sakurai, A., and Feldmann, K.A. (1998). The DWF4 gene of Arabidopsis encodes a cytochrome P450 that mediates multiple 22alpha-hydroxylation steps in brassinosteroid biosynthesis. Plant Cell 10: 231-243.

Choe, S., Fujioka, S., Noguchi, T., Takatsuto, S., Yoshida, S., and Feldmann, K. (2001). Overexpression of DWARF4 in the brassinosteroid biosynthetic pathway results in increased vegetative growth and seed yield in Arabidopsis. Plant J. 26: 573-582.

Clay, N.K., Adio, A.M., Denoux, C., Jander, G., and Ausubel, F.M. (2009). Glucosinolate metabolites required for an Arabidopsis innate immune response. Science 323: 95-101.

Collu, G., Unver, N., Peltenburg-Looman, A.M., van der Heijden, R., Verpoorte, R., and Memelink, J. (2001). Geraniol 10-hydroxylase, a cytochrome P450 enzyme involved in terpenoid indole alkaloid biosynthesis. FEBS Lett. 508: 215-220.

Compagnon, V., Diehl, P., Benveniste, I., Meyer, D., Schaller, H., Schreiber, L., Franke, R., and Pinot, F. (2009) CYP86B1 Is Required for Very Long Chain $\omega$-Hydroxyacid and $\alpha, \omega$-Dicarboxylic Acid Synthesis in Root and Seed Suberin Polyester. Plant Physiol. 120: 1831-1843.

Denoux, C., Galletti, R., Mammarella, N., Gopalan, S., Werck, D., De Lorenzo, G., Ferrari, S., Ausubel, F.M., and Dewdney, J. (2008). Activation of defense response pathways by OGs and Flg22 elicitors in Arabidopsis seedlings. Mol Plant. 1: 423-45.

De Vetten, N., Ter Horst, J., Van Schaik, H. P., De Boer, A., Mol, J., and Koes, R. (1999). A cytochrome $b 5$ is required for full activity of flavonoid 3',5'-hydroxylase, a cytochrome P450 involved in the formation of blue flower colors. Proc. Natl. Acad. Sci. USA 96: 778-783.

Delarue, M., Prinsen, E., Onckelen, H.V., Caboche, M., and Bellini, C. (1998). Sur2 mutations of Arabidopsis thaliana define a new locus involved in the control of auxin homeostasis. Plant J. 14: 603-611.

DeVos, M., and Jander, G. (2009). Myzus persicae (green peach aphid) salivary components induce defence responses in Arabidopsis thaliana. Plant Cell Environ. 32: 1548-1560.

Dobritsa, A.A., Shrestha, J., Morant, M., Pinot, F., Matsuno, M., Swanson, R., Møller, B.L., and Preuss, D. (2009). CYP704B1 is a longchain fatty acid omega-hydroxylase essential for sporopollenin synthesis in pollen of Arabidopsis. Plant Physiol. 151: 574-89.

Domínguez, E., Mercado, J.A., Quesada, M.A., and Heredia, A. (1999). Pollen sporopollenin: degradation and structural élucidation. Sex Plant Reprod. 12: 171-178.

Duan, H., Civjan, N.R., Sligar, S.G., and Schuler, M.A. (2004). Co-incorporation of heterologously expressed Arabidopsis cytochrome P450 and P450 reductase into soluble nanoscale lipid bilayers. Arch. Biochem. Biophys. 424: 141-153.

Duan, H., Huang, M.Y., Palacio, K., and Schuler, M.A. (2005) Variations in CYP74B2 (Hydroperoxide Lyase) Gene Expression Differentially Affect Hexenal Signaling in the Columbia and Landsberg erecta Ecotypes of Arabidopsis. Plant Physiol. 139: 1529-154

Duan, H., and Schuler, M.A. (2005) Differential Expression and Evolution of the Arabidopsis CYP86A Subfamily. Plant Physiol. 137: 1067-1081.

Durst, F., and Nelson, D. R. (1995). Diversity and evolution of plant P450 and P450-reductases. Drug Metab. Drug Interact. 12: 189-206.

Ehlting, J., Hamberger, B., Million-Rousseau, R. and Werck-Reichhart, D. (2006). Cytochromes P450 in phenolic metabolism. Phytochem. Rev. 5: 239-270.

Ehlting, J., Sauveplane, V., Olry, A., Ginglinger, J.F., Provart, N.J., and Werck-Reichhart, D. (2008). An extensive (co-)expression analysis tool for the cytochrome P450 superfamily in Arabidopsis thaliana. BMC Plant Biol. 28: 47.

Eriksson, S., Stransfeld, L., Adamski, N.M., Breuninger, H., and Lenhard, M. (2010). KLUH/CYP78A5-dependent growth signaling coordinates floral organ growth in Arabidopsis. Curr Biol. 20: 527-532.

Fellenberg, C., Böttcher, C., and Vogt, T. (2009). Phenylpropanoid polyamine conjugate biosynthesis in Arabidopsis thaliana flower buds. Phy- 
tochemistry 70: 1392-1400.

Ferro, M., Brugière, S., Salvi, D., Seigneurin-Berny, D., Court, M., Moyet, L., Ramus, C., Miras, S., Mellal, M., Le Gall, S., Kieffer-Jaquinod, S., Bruley, C., Garin, J., Joyard, J., Masselon, C., and Rolland, N. (2010) AT_CHLORO: A comprehensive chloroplast proteome database with subplastidial localization and curated information on envelope proteins. Mol. Cell. Proteomics 9: 1063-1084

Feyereisen, R. (2011) Arthropod CYPomes illustrate the tempo and mode in P450 evolution. Biochim. Biophys. Acta. 1814: 19-28.

Field, B., and Osbourn, A.E. (2008). Metabolic diversification--independent assembly of operon-like gene clusters in different plants. Science 320: 543-547.

Fiore, A., Dall'osto, L., Fraser, P.D., Bassi, R., and Giuliano, G. (2006). Elucidation of the beta-carotene hydroxylation pathway in Arabidopsis thaliana. FEBS Lett. 580: 4718-4722.

Flagel, L.E., and Wendel, J.F. (2009). Gene duplication and evolutionary novelty in plants. New Phytol. 183: 557-564.

Franke, R., Humphreys, J.M., Hemm, M.R., Denault, J.W., Ruegger, M.O., Cusumano, J.C., and Chapple, C. (2002a) The Arabidopsis REF8 gene encodes the 3-hydroxylase of phenylpropanoid metabolism. Plant J. 30: 33-45.

Franke, R., Hemm, M.R., Denault, J.W., Ruegger, M.O., Humphreys, J.M., and Chapple, C. (2002b). Changes in secondary metabolism and deposition of an unusual lignin in the ref8 mutant of Arabidopsis. Plant J. 30: 47-59.

Franke, R., McMichael, C.M., Meyer, K., Shirley, A.M., Cusumano, J.C., and Chapple, C. (2000). Modified lignin in tobacco and poplar plants over-expressing the Arabidopsis gene encoding ferulate 5-hydroxylase. Plant J. 22: 223-234.

Fraser, C.M., and Chapple, C. (2011). The Phenylpropanoid Pathway in Arabidopsis. The Arabidopsis Book 9: e0152. doi: 10.1199:tab.0152.

Frey, M., Chomet, P., Glawischnig, E., Stettner, C., Grün, S., Winklmair, A., Eisenreich, W., Bacher, A., Meeley, R.B., Briggs, S.P., Simcox, K., and Gierl, A. (1997). Analysis of a chemical plant defense mechanism in grasses. Science 277: 696-699.

Frey, M., Schullehner, K., Dick, R., Fiesselmann, A., and Gierl, A. (2009). Benzoxazinoid biosynthesis, a model for evolution of secondary metabolic pathways in plants. Phytochemistry 70: 1645-51.

Galbiati, M., Simoni, L., Pavesi, G., Cominelli, E., Francia, P., Vavasseur, A., Nelson, T., Bevan, M., and Tonelli, C. (2008) Genetrap lines identify Arabidopsis genes expressed in stomatal guard cells. Plant $\mathrm{J}$. 53: 750-762.

Galletti, R., Denoux, C., Gambetta, S., Dewdney, J., Ausubel, F.M., De Lorenzo, G., and Ferrari, S. (2008). The AtrbohD-mediated oxidative burst elicited by oligogalacturonides in Arabidopsis is dispensable for the activation of defense responses effective against Botrytis cinerea. Plant Physiol. 148: 1695-706.

Gesell, A., Rolf, M., Ziegler, J., Díaz Chávez, M.L., Huang, F.C., and Kutchan, T.M. (2009). CYP719B1 is salutaridine synthase, the C-C phenol-coupling enzyme of morphine biosynthesis in Opium Poppy. J. Biol. Chem. 284: 24432-24442.

Goda, H., Shimada, Y., Asami, T., Fujioka, S., and Yoshida, S. (2002). Microarray analysis of brassinosteroid-regulated genes in Arabidopsis. Plant Physiol. 130: 1319-1334.

Godiard, L., Sauviac, L., Dalbin, N., Liaubet, L., Callard, D., Czernic, P., and Marco, Y. (1998). CYP76C2, an Arabidopsis thaliana cytochrome P450 gene expressed during hypersensitive and developmental cell death. FEBS Lett. 438: 245-249.

Gomez-Roldan, V., Fermas, S., Brewer, P.B., Puech-Pages, V., Dun, E.A., Pillot, J.P., Letisse, F., Matusova, R., Danoun, S., Portais, J.C., Bouwmeester, H., Becard, G., Beveridge, C.A., Rameau, C., and
Rochange, S.F. (2008). Strigolactone inhibition of shoot branching Nature 455: 189-194.

Graham, S. E., and Peterson, J. A. (1999). How similar are P450s and what can their differences teach us. Arch. Bochem. Biophys. 369: 2429.

Greer, S., Wen, M., Bird, D., Wu, X., Samuels, L., Kunst, L., and Jetter, R. (2007). The cytochrome P450 enzyme CYP96A15 is the midchain alkane hydroxylase responsible for formation of secondary alcohols and ketones in stem cuticular wax of Arabidopsis. Plant Physiol. 145: 653-67.

Griebel, T., and Zeier, J. (2010). A role for beta-sitosterol to stigmasterol conversion in plant-pathogen interactions. Plant J. 63: 254-268.

Guengerich, F.P. (2007). Mechanisms of cytochrome P450 substrate oxidation: MiniReview. J. Biochem. Mol. Toxicol. 21: 163-168.

Hagemeier, J., Schneider, B., Oldham, N.J., and Hahlbrock, K. (2001). Accumulation of soluble and wall-bound indolic metabolites in Arabidopsis thaliana leaves infected with virulent or avirulent Pseudomonas syringae pathovar tomato strains. Proc. Natl. Acad. Sci. USA 98: 753758.

Halkier, B. A., Sibbesen, O., Koch, B., and Moller, B. L. (1995). Characterization of cytochrome P450TYR, a multifunctional haem-thiolate $\mathrm{N}$-hydroxylase involved in the biosynthesis of the cyanogenic glucoside dhurrin. Drug Metab. Drug Interact. 12: 285-297.

Halkier, B.A., and Gershenzon, J. (2006). Biology and biochemistry of glucosinolates. Annu Rev Plant Biol. 57:303-33.

Hallahan, D.L, Nugent, J.H., Hallahan, B.J., Dawson, G.W., Smiley, D.W., West, J.M., and Wallsgrove, R.M. (1992) Interactions of Avocado (Persea americana) Cytochrome P-450 with Monoterpenoids. Plant Physiol. 98: 1290-1297.

Hallahan, D.L., Lau, S.M., Harder, P.A., Smiley, D.W., Dawson, G.W., Pickett, J.A., Christoffersen, R.E., and O'Keefe, D.P. (1994). Cytochrome P-450-catalysed monoterpenoid oxidation in catmint (Nepeta racemosa) and avocado (Persea americana); evidence for related enzymes with different activities. Biochim. Biophys. Acta 1201: 94-100.

Hamberger, B., and Bohlmann, J. (2006). Cytochrome P450 mono-oxygenases in conifer genomes: discovery of members of the terpenoid oxygenase superfamily in spruce and pine. Biochem. Soc. Trans. 34: 1209-1214.

Hamdane, D., Zhang, H., and Hollenberg, P. (2008). Oxygen activation by cytochrome P450 monooxygenase. Photosynth. Res. 98: 657-666.

Hannemann, F., Bichet, A., Ewen, K.M., and Bernhardt, R. (2007). Cytochrome P450 systems-biological variations of electron transport chains. Biochim. Biophys. Acta 1770: 330-344.

Hansen, C.H., Du, L., Naur, P., Olsen, C.E., Axelsen, K.B., Hick, A.J., Pickett, J.A., and Halkier, B.A. (2001). CYP83B1 is the oxime-metabolizing enzyme in the glucosinolate pathway in Arabidopsis. J. Biol Chem. 276: 24790-24796.

Hansen, C.H., Wittstock, U., Olsen, C.E., Hick, A.J., Pickett, J.A., and Halkier, B.A. (2001). Cytochrome P450 CYP79F1 from Arabidopsis catalyzes the conversion of dihomomethionine and trihomomethionine to the corresponding aldoximes in the biosynthesis of aliphatic glucosinolates. J. Biol. Chem. 276: 11078-11085.

Hasemann, C.A., Kurumbail, R.G., Boddupalli, S.S., Peterson, J.A., and Deisenhofer, J. (1995). Structure and function of cytochromes P450: a comparative analysis of thee crystal structures. Structure 3: 41-62.

Haudenschild, C., Schalk, M., Karp, F., and Croteau, R. (2000). Functional expression of regiospecific cytochrome P450 limonene hydroxylases from mint (Mentha spp.) in Escherichia coli and saccharomyces cerevisiae. Arch. Biochem. Biophys. 379: 127-136.

He, J.-X., Gendron, J.M., Sun, Y., Gampala, S.S., Gendron, N., Sun, 
C.Q., and Wang, Z.-Y. (2005). BZR1 is a transcriptional repressor with dual roles in brassinosteroid homeostasis and growth responses. Science 307: 1634-1638.

Helliwell, C.A., Sheldon, C.C., Olive, M.R., Walker, A.R., Zeevaart, J. A., Peacock, W.J., and Dennis, E.S. (1998). Cloning of the Arabidopsis ent-kaurene oxidase gene GA3. Proc. Natl. Acad. Sci. USA 95: 9019-9024.

Helliwell, C.A., Poole, A., Peacock, W.J., and Dennis, E.S. (1999). Arabidopsis ent-kaurene oxidase catalyzes three steps of gibberellin biosynthesis. Plant Physiol. 119: 507-510.

Helliwell, C.A., Chandler, P.M., Poole, A., Dennis, E.S., and Peacock, W.J. (2001a). The CYP88A cytochrome P450, ent-kaurenoic acid oxidase, catalyzes three steps of the gibberellin biosynthesis pathway. Proc. Natl. Acad. Sci. USA 98: 2065-2070.

Helliwell, C.A., Sullivan, J.A., Mould, R.M., Gray, J.C., Peacock, W.J., and Dennis, E.S. (2001b). A plastid envelope location of Arabidopsis ent-kaurene oxidase links the plastid and endoplasmic reticulum steps of the gibberellin biosynthesis pathway. Plant J. 28: 201-208.

Hemm, M.R., Rider, S.D., Ogas, J., Murry, D.J., and Chapple, C. (2004). Light induces phenylpropanoid metabolism in Arabidopsis roots. Plant J. 38: 765-778.

Hemm, M.R., Ruegger, M.O., and Chapple, C. (2003). The Arabidopsis ref2 mutant is defective in the gene encoding CYP83A1 and shows both phenylpropanoid and glucosinolate phenotypes. Plant Cell. 15:179-194.

Hirano, K., Nakajima, M., Asano, K., Nishiyama, T., Sakakibara, H., Kojima, M., Katoh, E., Xiang, H., Tanahashi, T., Hasebe, M., Banks, J.A., Ashikari, M., Kitano, H., Ueguchi-Tanaka, M., and Matsuoka, M. (2007). The GID1-Mediated Gibberellin Perception Mechanism Is Conserved in the Lycophyte Selaginella moellendorffii but Not in the Bryophyte Physcomitrella patens. Plant Cell 19: 3058-79.

Hoecker, U., Toledo-Ortiz, G., Bender, J., and Quail, P.H. (2004). The photomorphogenesis-related mutant red1 is defective in CYP83B1, a red light-induced gene encoding a cytochrome P450 required for normal auxin homeostasis. Planta 219: 195-200.

Höfer, R., Briesen, I., Beck, M., Pinot, F., Schreiber, L., and Franke, R. (2008). The Arabidopsis cytochrome P450 CYP86A1 encodes a fatty acid $\omega$-hydroxylase involved in suberin monomer biosynthesis. J. Exp. Bot. 59: 2347-2360.

Holton, T.A., Brugliera, F., Lester, D.R., Tanaka, Y., Hyland, C.D., Menting, J.G., Lu, C.Y., Farcy, E., Stevenson, T.W., and Cornish, E.C. (1993). Cloning and expression of cytochrome P450 genes controlling flower colour. Nature 366: 276-279.

Huang, J., Bhinu, V.S., Li X., Dallal Bashi, Z., Zhou, R., and Hannoufa, A. (2009). Pleiotropic changes in Arabidopsis f5h and sct mutants revealed by large-scale gene expression and metabolite analysis. Planta 230: 1057-1069.

Hughes, R.K., Belfield, E.J., Ashton, R., Fairhurst, S.A., Göbel, C., Stumpe, M., Feussner, I., and Casey, R. (2006). Allene oxide synthase from Arabidopsis thaliana (CYP74A1) exhibits dual specificity that is regulated by monomer-micelle association. FEBS Lett. 580: 4188-4194.

Hull, A.K., Vij, R., and Celenza, J.L. (2000). Arabidopsis cytochrome $\mathrm{P} 450$ s that catalyze the first step of tryptophan-dependent indole3-acetic acid biosynthesis. Proc. Natl. Acad. Sci. USA 97: 2379-2384.

Huntley, S.K., Ellis, D., Gilbert, M., Chapple, C., and Mansfield, S.D. (2003). Significant increases in pulping efficiency in C4H-F5H-transformed poplars: improved chemical savings and reduced environmental toxins. J. Agric. Food Chem. 51: 6178-6183.

Humphreys, J.M., Hemm, M.R., and Chapple, C. (1999). New routes for lignin biosynthesis defined by biochemical characterization of recombi- nant ferulate 5-hydroxylase, a multifunctional cytochrome P450-dependent monooxygenase. Proc. Natl. Acad. Sci. USA 96: 10045-10050.

Ikezawa, N., Tanaka, M., Nagayoshi, M., Shinkyo, R., Sakaki, T., Inouye, K., and Sato, F. (2003). Molecular cloning and characterization of CYP719, a methylenedioxy bridge-forming enzyme that belongs to a novel P450 family, from cultured Coptis japonica cells. J. Biol. Chem. 278: 38557-38565.

Imaishi, H., Matsuo, S., Swai, E., and Ohkawa, H. (2000). CYP78A1 preferentially expressed in developing inflorescences of Zea mays encoded a cytochrome P450-dependent lauric acid 12-monooxygenase. Biosci. Biotechnol. Biochem. 64: 1696-1701.

Irmler, S., Schroder, G., St-Pierre, B., Crouch, N.P., Hotze, M., Schmidt, J., Strack, D., Matern, U., and Schroder, J. (2000). Indole alkaloid biosynthesis in Catharanthus roseus: new enzyme activities and identification of cytochrome P450 CYP72A1 as secologanin synthase. Plant J. 24: 797-804.

Isin, E.M., and Guengerich, F.P. (2007). Complex reactions catalyzed by cytochrome P450 enzymes. Biochim. Biophys. Acta 1770: 314-329.

Ito, T., and Meyerowitz, E. M. (2000). Overexpression of a gene encoding a cytochrome P450, CYP78A9, induces large and seedless fruit in Arabidopsis. Plant Cell 12: 1541-1550.

Jensen, K., and Møller, B.L. (2010). Plant NADPH-cytochrome P450 oxidoreductases. Phytochemistry 71:132-141.

Jiang, H., and Morgan J.A. (2003). Optimization of an in vivo plant P450 monooxygenase system in Saccharomyces cerevisiae. Biotech. Bioeng. 85: 130-137.

Jin, H., Cominelli, E., Bailey, P., Parr, A., Mehrtens, F., Jones, J., Tonelli, C., Weisshaar, B., and Martin, C. (2000). Transcriptional repression by AtMYB4 controls production of UV-protecting sunscreens in Arabidopsis. EMBO J. 19: 6150-6161.

Kai, K., Hashidzume, H., Yoshimura, K., Suzuki, H., Sakurai, N., Shibata, D., and Ohta, D. (2009). Metabolomics for the characterization of cytochromes P450-dependent fatty acid hydroxylation reactions in Arabidopsis. Plant Biotechnol. 26: 175-182.

Kandel, S., Morant, M., Benveniste, I., Blée, E., Werck-Reichhart, D., and Pinot, F. (2005). Cloning, functional expression, and characterization of CYP709C1, the first sub-terminal hydroxylase of long chain fatty acid in plants. Induction by chemicals and methyl jasmonate. J. Biol. Chem. 280: 35881-35889.

Kandel, S., Sauveplane, V., Compagnon, V., Franke, R., Millet, Y., Schreiber, L., Werck-Reichhart, D., and Pinot, F. (2007). Characterization of a methyl jasmonate and wounding-responsive cytochrome P450 of Arabidopsis thaliana catalyzing dicarboxylic fatty acid formation in vitro. FEBS J. 274: 5116-5127.

Kannangara, R., Branigan, C., Liu, Y., Penfield, T., Rao, V., Mouille, G., Höfte, H., Pauly, M., Riechmann, J.L., and Broun, P. (2007). The Transcription Factor WIN1/SHN1 Regulates Cutin Biosynthesis in Arabidopsis thaliana. Plant Cell 19: 1278-1294.

Kappers, I.F., Aharoni, A., van Herpen, T.W., Luckerhoff, L.L., Dicke, M., and Bouwmeester, H.J. (2005) Genetic engineering of terpenoid metabolism attracts bodyguards to Arabidopsis. Science. 309: 2070-2.

Kawakami, N. (2006). Isolation and characterization of high temperatureresistant germination mutants of Arabidopsis thaliana. Plant Cell Physiol. 47: 1081-1094.

Kerhoas, L., Aouak, D., Cingöz, A., Routaboul, J.M., Lepinie, L., Einhorn, J., and Birlirakis, N. (2006). Structural characterization of the major flavonoid glycosides from Arabidopsis thaliana seeds. J. Agric. Food Chem. 54: 6603-6612.

Kim, G.T., Tsukaya, H., and Uchimiya, H. (1998). The ROTUNDIFOLIA3 gene of Arabidopsis thaliana encodes a new member of the cytochrome P-450 family that is required for the regulated polar elongation 
of leaf cells. Genes Dev. 12: 2381-2391

Kim, G.T., Tsukaya, H., Saito, Y., and Uchimiya, H. (1999) Changes in the shapes of leaves and flowers upon overexpression of cytochrome P450 in Arabidopsis. Proc. Natl. Acad. Sci. USA. 3: 9433-7.

Kim, H.B., Schaller, H., Goh, C.H., Kwon, M., Choe, S., An, C.S., Durst, F., Feldmann, K.A., and Feyereisen, R. (2005a). Arabidopsis cyp51 mutant shows postembryonic seedling lethality associated with lack of membrane integrity. Plant Physiol. 138: 2033-2047.

Kim, T.W., Hwang, J.Y., Kim, Y.S., Joo, S.H,, Chang, S.C., Lee, J.S., Takatsuto, S., and Kim, S.K. (2005b). Arabidopsis CYP85A2, a cytochrome P450, mediates the Baeyer-Villiger oxidation of castasterone to brassinolide in brassinosteroid biosynthesis. Plant Cell 17: 2397-2412.

Kim, G.T., Fujioka, S., Kozuka, T., Tax, F.E., Takatsuto, S., Yoshida, S., and Tsukaya, H. (2005c). CYP90C1 and CYP90D1 are involved in different steps in the brassinosteroid biosynthesis pathway in Arabidopsis thaliana. Plant J. 41: 710-721.

Kim, T.W., Guan, S.H., Sun, Y., Deng, Z.P., Tang, W.Q., Shang, J.X., Sun, Y., Burlingame, A.L., and Wang, Z.Y. (2009). Brassinosteroid signal transduction from cell-surface receptor kinases to nuclear transcription factors. Nat. Cell Biol. 11: 1254-1233.

Kim, J., and DellaPenna, D. (2006). Defining the primary route for lutein synthesis in plants: the role of Arabidopsis carotenoid beta-ring hydroxylase CYP97A3. Proc. Natl. Acad. Sci. USA 103: 3474-3479.

Kim, J.E., Cheng, K.M., Craft, N.E., Hamberger, B., and Douglas, C.J. (2010). Over-expression of Arabidopsis thaliana carotenoid hydroxylases individually and in combination with a beta-carotene ketolase provides insight into in vivo functions. Phytochemistry 71: 168-178.

Kim, J.H., and Jander, G. (2007). Myzus persicae (green peach aphid) feeding on Arabidopsis induces the formation of a deterrent indole glucosinolate. Plant J. 49: 1008-1019.

Kohlen, W., Charnikhova, T., Liu, Q., Bours, R., Domagalska, M.A., Beguerie, S., Verstappen, F., Leyser, O., Bouwmeester, H., and Ruyter-Spira, C. (2011) Strigolactones are transported through the xylem and play a key role in shoot architectural response to phosphate deficiency in nonarbuscular mycorrhizal host Arabidopsis. Plant Physiol. Plant Physiol. 155: 974-987.

Koichi, Y., Xiaonan, X., Kaori, Y., and Yasutomo, T. (2009). Strigolactones: structures and biological activities. Pest Management Sci. 65: 467-470.

Koornneef, M., and Vanderveen, J.H. (1980). Induction and analysis of gibberellin sensitive mutants in Arabidopsis thaliana (L) heynh. Theor. Appl. Genet. 58: 257-263.

Koornneef, M. (1982). A gene controling flavonoid 3'-hydroxylation in Arabidopsis. Arabidopsis Information Service 19: 113-115.

Kruse, T., Ho, K., Yoo, H.D., Johnson, T., Hippely, M., Park, J.H., Flavell, R., and Bobzin, S. (2008). In planta biocatalysis screen of P450s identifies 8-methoxypsoralen as a substrate for the CYP82C subfamily, yielding original chemical structures. Chem. Biol. 15: 149-156.

Kubigsteltig, I., Laudert, D., and Weiler, E. W. (1999). Structure and regulation of the Arabidopsis thaliana allene oxide synthase gene. Planta 208: 463-471.

Kushiro, T., Okamoto, M., Nakabayashi, K., Yamagishi, K., Kitamura, S., Asami, T., Hirai, N., Koshiba, T., Kamiya, Y., and Nambara, E. (2004). The Arabidopsis cytochrome P450 CYP707A encodes ABA 8‘-hydroxylases: key enzymes in ABA catabolism. EMBO J. 23: 16471656.

Kutchan, T.M. (1996). Heterologous expression of alkaloid biosynthetic genes-a review. Gene 179: 73-81.

Kwon, M., Fujioka, S., Jeon, J.H., Kim, H.B., Takatsuto, S., Yoshida, S., An, C.S., and Choe, S. (2005). A double mutant for the CYP85A1 and CYP85A2 genes of Arabidopsis exhibits a brassinosteroid dwarf phenotype. J. Plant Biol. 48: 237-244.

Larbat, R., Kellner, S., Specker, S., Hehn, A., Gontier, E., Hans, J., Bourgaud, F., and Matern, U. (2007) Molecular cloning and functional characterization of psoralen synthase, the first committed monooxygenase of furanocoumarin biosynthesis. J Biol Chem. 5: 542-54.

Latunde-Dada, A.O., Cabello-Hurtado, F., Czittrich, N., Didierjean, L., Schopfer, C., Hertkorn, N., Werck-Reichhart, D., and Ebel, J. (2001). Flavonoid 6-hydroxylase from soybean (Glycine max L.), a novel plant P-450 monooxygenase. J. Biol. Chem. 276: 1688-1695.

Landry, L.G., Chapple, C.C., and Last, R.L. (1995). Arabidopsis mutants lacking phenolic sunscreens exhibit enhanced ultraviolet-B injury and oxidative damage. Plant Physiol. 109: 1159-1166.

Laudert, D., Schaller, F., and Weiler, E.W. (2000). Transgenic Nicotiana tabacum and Arabidopsis thaliana plants overexpressing allene oxide synthase. Planta 211: 163-165.

Laudert, D., and Weiler, E.W. (1998). Allene oxide synthase: a major control point in Arabidopsis thaliana octadecanoid signalling. Plant $\mathrm{J}$. 15: 675-684.

Le Bouquin, R., Skrabs, M., Kahn, R., Benveniste, I., Salaün, J.P., Schreiber, L., Durst, F., and Pinot, F. (2001). CYP94A5, a new cytochrome P450 from Nicotiana tabacum is able to catalyze the oxidation of fatty acids to the $\omega$-alcohol and to the corresponding diacid. Eur. J. Biochem. 268: 3083-3090.

Lee, D.S., Nioche, P., Hamberg, M., and Raman, C.S. (2008). Structural insights into the evolutionary paths of oxylipin biosynthetic enzymes. Nature 455: 363-370.

Lee, S, Badieyan, S., Bevan, D.R., Herde, M., Gatz, C., and Tholl, D. (2010). Herbivore-induced and floral homoterpene volatiles are biosynthesized by a single P450 enzyme (CYP82G1) in Arabidopsis. Proc. Natl. Acad. Sci. USA 107: 21205-21210.

Li, Y., Beisson, F., Koo, A.J.K., Molina, I., Pollard, M., and Ohlrogge, J. (2007). Identification of acyltransferases required for cutin biosynthesis and production of cutin with suberin-like monomers. Proc. Natl. Acad. Sci. USA 104: 18339-18344.

Li-Beisson, Y., Pollard, M., Sauveplane, V., Pinot, F., Ohlrogge, J., and Beisson, F. (2009). Nanoridges that characterize the surface morphology of flowers require the synthesis of cutin polyester. Proc. Natl. Acad. Sci. USA 106: 22008-22013.

Liu, F., Jiang, H., Ye, S., Chen, W.P., Liang, W., Xu, Y., Sun, B., Sun, J., Wang, Q., Cohen, J.D., and Li, C. (2010); The Arabidopsis P450 protein CYP82C2 modulates jasmonate-induced root growth inhibition, defense gene expression and indole glucosinolate biosynthesis. Cell Res. 20: 539-552.

Long, M., Rosenberg, C., and Gilbert, W. (1995). Intron phase correlation and the evolution of the intron/exon structures of genes. Proc. Natl. Acad. Sci. USA 92: 12495-12499.

Luo, P., Wang, Y.H., Wang, G.D., Essenberg, M., and Chen, X.Y. (2001). Molecular cloning and functional identification of (+)-delta-cadinene8-hydroxylase, a cytochrome P450 mono-oxygenase (CYP706B1) of cotton sesquiterpene biosynthesis. Plant J. 28: 95-104.

Ma, H., Zhang, S., Ji, L., Zhu, H ., Yang, S., Fang, X., and Yang, R. (2006). Fine mapping and in silico isolation of the EU/1 gene controlling upper internode elongation in rice. Plant Mol. Biol. 60: 87-94.

Mansuy, D. (1998). The great diversity of reactions catalyzed by cytochrome P450. Comp. Biochem. Physiol. Part. C. 121: 5-14

Marita, J.M., Ralph, J., Hatfield, R.D., and Chapple, C. (1999). NMR characterization of lignins in Arabidopsis altered in the activity of ferulate 5-hydroxylase. Proc. Natl. Acad. Sci. USA 96: 12328-12332.

Mathur, J., Molnar, G., Fujioka, S., Takatsuto, S., Sakurai, A., Yokota, T., Adam, G., Voigt, B., Nagy, F., Maas, C., Schell, J., Koncz, C., and Szekeres, M. (1998). Transcription of the Arabidopsis CPD gene, 
encoding a steroidogenic cytochrome $\mathrm{P} 450$, is negatively controlled by brassinosteroids. Plant J. 14: 593-602.

Matsuno, M., Compagnon, V., Schoch, G.A., Schmitt, M., Debayle, D., Bassard, J.E., Pollet, B., Hehn, A., Heintz, D., Ullmann, P., Lapierre, C., Bernier, F., Ehlting, J., and Werck-Reichhart, D. (2009). Evolution of a novel phenolic pathway for pollen development. Science 325: 1688-1692.

Mehrtens, F., Kranz, H., Bednarek, P., and Weisshaar, B. (2005). The Arabidopsis transcription factor MYB12 is a flavonol-specific regulator of phenylpropanoid biosynthesis. Plant Physiol. 138: 1083-1096.

Meyer, K., Cusumano, J., Somerville, C., and Chapple, C. (1996). Ferulate-5-hydroxylase from Arabidopsis thaliana defines a new family of cytochrome P450-dependent monoxygenases. Proc. Natl. Acad. Sci. USA 93: 6869-6874.

Meyer, K., Shirley, A.M., Cusumano, J.C., Bell-Lelong, D.A., and Chapple, C. (1998). Lignin monomer composition is determined by the expression of a cytochrome P450-dependent monooxygenase in Arabidopsis. Proc. Natl. Acad. Sci. USA 95: 6619-6623.

Mikkelsen, M.D., Hansen, C.H., Wittstock, U., and Halkier, B.A. (2000). Cytochrome P450 CYP79B2 from Arabidopsis catalyzes the conversion of tryptophan to indole-3-acetaldoxime, a precursor of indole glucosinolates and indole-3-acetic acid. J. Biol. Chem. 275: 33712-33717.

Mikkelsen, M.D., and Halkier, B.A. (2003). Metabolic engineering of valine- and isoleucine-derived glucosinolates in Arabidopsis expressing CYP79D2 from cassava. Plant Physiol. 131: 773-779.

Millet, Y.A., Danna, C.H., Clay, N.K., Songnuan, W., Simon, M.D., Werck-Reichhart, D., and Ausubel, F.M. (2010). Innate immune responses activated in Arabidopsis roots by microbe-associated molecular patterns. Plant Cell 22: 973-990.

Mizutani, M., and Ohta, D. (1998). Two isoforms of NADPH:cytochrome P450 reductase in Arabidopsis thaliana. Gene structure, heterologous expression in insect cells, and differential regulation. Plant Physiol. 116: 357-367.

Mizutani, M., Ohta, D., and Sato, R. (1997). Isolation of a cDNA and a genomic clone encoding cinnamate 4-hydroxylase from Arabidopsis and its expression manner in planta. Plant Physiol. 113: 755-763.

Mizutani, M., Ward, E., and Ohta, D. (1998). Cytochrome P450 superfamily in Arabidopsis thaliana: isolation of cDNAs, differential expression, and RFLP mapping of multiple cytochromes P450. Plant Mol. Biol. 37: 39-52.

Mizutani, M., and Ohta, D. (2010). Diversification of P450 Genes During Land Plant Evolution. Annu. Rev. Plant Biol. 61: 291-315.

Molina, I., Ohlrogge, J.B., and Pollard, M. (2008). Deposition and localization of lipid polyester in developing seeds of Brassica napus and Arabidopsis thaliana. Plant J. 53: 437-449.

Molina, I., Li-Beisson, Y., Beisson, F., Ohlrogge, J.B., and Pollard, M. (2009). Identification of an Arabidopsis Feruloyl-Coenzyme A Transferase Required for Suberin Synthesis. Plant Physiol. 151: 1317-1328.

Morant, M., Bak, S., Møller, B.L., and Werck-Reichhart, D. (2003). Plant cytochromes P450: tools for pharmacology, plant protection and phytoremediation. Curr. Opin .Biotechnol. 14: 151-62.

Morant, M., Jørgensen, K., Schaller, H., Pinot, F., Møller, B.L., WerckReichhart, D., and Bak, S. (2007). CYP703 is an ancient cytochrome P450 in land plants catalyzing in-chain hydroxylation of lauric acid to provide building blocks for sporopollenin synthesis in pollen. Plant Cell 19: $1473-87$

Morant, M., Ekstrøm, C., Ulvskov, P., Kristensen, C., Rudemo, M., Olsen, C.E., Hansen, J., Jørgensen, K., Jørgensen, B., Møller, B.L., and Bak, S. (2010). Metabolomic, transcriptional, hormonal, and signaling cross-talk in Superroot2. Mol. Plant 21: 192-211.

Morikawa, T., Mizutani, M., Aoki, N., Watanabe, B., Saga, H., Saito,
S., Oikawa, A., Suzuki, H., Sakurai, N., Shibata, D., Wadano, A., Sakata, K., and Ohta, D. (2006). Cytochrome P450 CYP710A encodes the sterol C-22 desaturase in Arabidopsis and Tomato. Plant Cell 18: 1008-1022.

Morikawa, T., Saga, H., Hashizume, H., and Ohta, D. (2009). CYP710A genes encoding sterol C22-desaturase in Physcomitrella patens as molecular evidence for the evolutionary conservation of a sterol biosynthetic pathway in plants. Planta 229: 1311-1322.

Murphy, P.J., and West, C.A. (1969). The role of mixed function oxidases in kaurene metabolism in Echinocystis macrocarpa Greene endosperm. Arch. Biochem. Biophys. 133: 395-407.

Nakamura, M., Satoh, T., Tanaka, S.I., Mochizuki, N., Yokota, T., and Nagatani, A. (2005). Activation of the cytochrome P450 gene, CY$P 72 C 1$, reduces the levels of active brassinosteroids in vivo. J. Exp. Bot. 56: 833-840.

Nafisi, M., Goregaoker, S., Botanga, C.J., Glawischnig, E., Olsen, C.E., Halkier, B.A., and Glazebrook, J. (2007). Arabidopsis cytochrome P450 monooxygenase 71A13 catalyzes the conversion of indole-3-acetaldoxime in camalexin synthesis. Plant Cell 19: 2039-2052.

Naur, P., Petersen, B.L., Mikkelsen, M.D., Bak, S., Rasmussen, H., Olsen, C.E., and Halkier, B.A. (2003). CYP83A1 and CYP83B1, two nonredundant cytochrome P450 enzymes metabolizing oximes in the biosynthesis of glucosinolates in Arabidopsis. Plant Physiol. 133: 63-72.

Neff, M.M., Nguyen, S.M., Malancharuvil, E.J., Fujioka, S., Noguchi, T., Seto, H., Tsubuki, M., Honda, T., Takatsuto, S., Yoshida, S., and Chory, J. (1999). BAS1: A gene regulating brassinosteroid levels and light responsiveness in Arabidopsis. Proc. Natl. Acad. Sci. USA 96: 15316-15323

Nelson, D.R., Schuler, M.A., Paquette, S.M., Werck-Reichhart, D., and Bak, S. (2004). Comparative genomics of Rice and Arabidopsis. Analysis of 727 cytochrome P450 genes and pseudogenes from a monocot and a dicot. Plant Physiol. 135: 756-772.

Nelson, D.R. (1999). Cytochrome P450 and the individuality of species. Arch. Biochem. Biophys. 369: 1-10.

Nelson, D.R., Koymans, L., Kamataki, T., Stegeman, J.J., Feyereisen, R., Waxman, D.J., Waterman, M.R., Gotoh, O., Coon, M.J., Estabrook, R.W., Gunsalus, I.C., and Nebert, D.W. (1996). P450 superfamily: update on new sequences, gene mapping, accession numbers and nomenclature. Pharmacogenetics 6: 1-42.

Nelson, D.R. (2006a). Cytochrome P450 nomenclature 2004. Methods Mol. Biol. 320: 1-10.

Nelson, D.R. (2006b). Plant cytochrome P450s from moss to poplar. Phytochemistry Rev. 5: 193-204.

Nelson, D.R., Ming, R., Alam, M., and Schuler, M.A. (2008). Comparison of cytochrome P450 genes from six plant genomes. Tropical Plant Biol. 1: 216-235.

Nelson, D.R. (2009). The cytochrome P450 homepage. Hum. Genomics 4: 59-65.

Nelson, D.R., and Werck-Reichhart, D. (2011). A P450 centric view of plant evolution. Plant J. 66: 194-211.

Noguchi, T., Fujioka, S., Choe, S., Takatsuto, S., Yoshida, S., Yuan, H., Feldmann, K.A., and Tax, F.E. (1999). Brassinosteroid-insensitive dwarf mutants of Arabidopsis accumulate brassinosteroids. Plant Physiol. 121: 743-752.

Nomura, T., Kushiro, T., Yokota, T., Kamiya, Y., Bishop, G.J., and Yamaguchi, S. (2005). The last reaction producing brassinolide is catalyzed by cytochrome P-450s, CYP85A3 in Tomato and CYP85A2 in Arabidopsis. J. Biol. Chem. 280: 17873-17879.

Normanly, J., and Bartel, B. (1999). Redundancy as a way of life - IAA metabolism. Curr. Opin. Plant Biol. 2: 207-213.

Ohnishi, T., Szatmari, A.M., Watanabe, B., Fujita, S., Bancos, S., 
Koncz, C., Lafos, M., Shibata, K., Yokota, T., Sakata, K., Szekeres, M., and Mizutani, M. (2006a). C-23 hydroxylation by Arabidopsis CYP90C1 and CYP90D1 reveals a novel shortcut in brassinosteroid biosynthesis. Plant Cell 18: 3275-3288.

Ohnishi, T., Nomura, T., Watanabe, B., Ohta, D., Yokota, T., Miyagawa, H., Sakata, K., and Mizutani, M. (2006b). Tomato cytochrome P450 CYP734A7 functions in brassinosteroid catabolism. Phytochemistry 67: 1895-1906.

Ohnishi, T., Watanabe, B., Sakata, K., and Mizutani, M. (2006c) CYP724B2 and CYP90B3 function in the early C-22 hydroxylation steps of brassinosteroid biosynthetic pathway in Tomato. Biosci. Biotech. Biochem. 70: 2071-2080.

Ohta, D., and Mizutani, M. (1998). Plant geraniol/nerol 10-hydroxylase and DNA coding therefor. US Patent No. 5753507.

Ohta, D., Kanaya, S., and Suzuki, H. (2010). Application of Fourier-transform ion cyclotron resonance mass spectrometry to metabolic profiling and metabolite identification. Curr. Opin. Biotechnol. 21: 35-44.

Okamoto, M., Kuwahara, A., Seo, M., Kushiro, T., Asami, T., Hirai, N., Kamiya, Y., Koshiba, T., and Nambara, E. (2006). CYP707A1 and CYP707A2, which encode abscisic acid 8 '-hydroxylases, are indispensable for proper control of seed dormancy and germination in Arabidopsis. Plant Physiol. 141: 97-107.

Okamoto, M., Tanaka, Y., Abrams, S.R., Kamiya, Y., Seki, M., and Nambara, E. (2009). High humidity induces abscisic acid 8 '-hydroxylase in stomata and vasculature to regulate local and systemic abscisic acid responses in Arabidopsis. Plant Physiol. 149: 825-834.

Olry, A., Schneider-Balhaddad, F., Heintz, D., and Werck-Reichhart, D. (2007). A medium throughput screening assay to determine cataIytic activities of oxygen-consuming enzymes: a new tool for functional characterization of cytochromes P450 and other oxygenases. Plant J. 51: 331-340.

Omura, T., and Sato, R. (1964). The carbon monoxide-binding pigment of liver microsomes. I. Evidence for its hemoprotein nature. J. Biol. Chem. 239: 2370-2378.

Osbourn, A.E., and Field, B. (2009). Operons. Cell Mol. Life Sci. 66: 3755-7375.

Pan, Y., Michael, T.P., Hudson, M.E., Kay, S.A., Chory, J., and Schuler, M.A. (2009). Cytochrome P450 monooxygenases as reporters for circadian-regulated pathways. Plant Physiol. 150: 858-878.

Paquette, S.M., Bak, S., and Feyereisen, R. (2000). Intron-exon organization and phylogeny in a large superfamily, the paralogous cytochrome P450 genes of Arabidopsis thaliana. DNA Cell Biol. 19: 307-317.

Paquette, S.M., Jensen, K., and Bak, S. (2009). A web-based resource for the Arabidopsis P450, cytochromes b5, NADPH-cytochrome P450 reductases, and family 1 glycosyltransferases (http://www.P450.kvl.dk). Phytochemistry 70: 1940-7.

Park, J.H., Halitschke, R., Kim, H.B., Baldwin, I.T., Feldmann, K.A., and Feyereisen, R. (2002). A knock-out mutation in allene oxide synthase results in male sterility and defective wound signal transduction in Arabidopsis due to a block in jasmonic acid biosynthesis. Plant $\mathrm{J}$. 31: 1-12.

Patten, A.M., Jourdes, M., Cardenas, C.L., Laskar, D.D., Nakazawa, Y.B, Chung, B.Y., Franceschi, V.R., Davin, L.B., and Lewis, N.G. (2010). Probing native lignin macromolecular configuration in Arabidopsis thaliana in specific cell wall types: further insights into limited substrate degeneracy and assembly of the lignins of ref8, fah 1-2 and C4H::F5H lines. Mol. Biosyst. 6: 499-515.

Pfalz, M., Vogel, H., and Kroymann, J. (2009). The gene controlling the indole glucosinolate modifier1 quantitative trait locus alters indole glucosinolate structures and aphid resistance in Arabidopsis. Plant Cell 21: 985-999.
Ping, L., Yan-Hong, W., Guo-Dong, W., Margaret, E., and Xiao-Ya, C. (2001). Molecular cloning and functional identification of (+)- $\delta$-cadinene8-hydroxylase, a cytochrome P450 mono-oxygenase (CYP706B1) of cotton sesquiterpene biosynthesis. Plant J. 28: 95-104.

Powles, S.B., and Yu, Q. (2010). Evolution in Action: Plants Resistant to Herbicides. Annu Rev Plant Biol. 61:317-347.

Qi, X., Bakht, S., Leggett, M., Maxwell, C., Melton, R., and Osbourn, A. (2004). A gene cluster for secondary metabolism in oat: implications for the evolution of metabolic diversity in plants. Proc. Natl. Acad. Sci. USA 101: 8233-8238.

Qi, X., Bakht, S., Qin, B., Leggett, M., Hemmings, A., Mellon, F., Eagles, J., Werck-Reichhart, D., Schaller, H., Lesot, A., Melton, R., and Osbourn, A. (2006). A different function for a member of an ancient and highly conserved cytochrome P450 family: from essential sterols to plant defense. Proc. Natl. Acad. Sci. USA 103: 18848-18853.

Reinhardt, B., Hanggi, E., Muller, S., Bauch, M., Wyrzykowska, J., Kerstetter, R., Poethig, S., and Fleming, A.J. (2007). Restoration of DWF4 expression to the leaf margin of a dwf4 mutant is sufficient to restore leaf shape but not size: the role of the margin in leaf development. Plant J. 52: 1094-1104.

Reintanz, B., Lehnen, M., Reichelt, M., Gershenzon, J., Kowalczyk, M., Sandberg, G., Godde, M., Uhl, R., and Palme, K. (2001). bus, a Bushy arabidopsis CYP79F1 knockout mutant with abolished synthesis of short chain aliphatic glucosinolates. Plant Cell 13: 351-367.

Rezen, T., Debeljak, N., Kordis, D., and Rozman, D. (2004). New aspects on lanosterol 14alpha-demethylase and cytochrome P450 evolution: lanosterol/cycloartenol diversification and lateral transfer. J. Mol. Evol. 59: 51-8.

Ro, D.K., Ehlting, J., Keeling, C.I., Lin, R., Mattheus, N., and Bohlmann, J. (2006). Microarray expression profiling and functional characterization of AtTPS genes: duplicated Arabidopsis thaliana sesquiterpene synthase genes At4g13280 and At4g13300 encode root-specific and wound-inducible (Z)-gamma-bisabolene synthases. Arch. Biochem. Biophys. 448: 104-116.

Rohloff, J., and Bones, A.M. (2005). Volatile profiling of Arabidopsis thaliana - putative olfactory compounds in plant communication. Phytochemistry $66: 1941-1955$.

Rontein, D., Onillon, S., Herbette, G., Lesot, A., Werck-Reichhart, D., Sallaud, C., and Tissier, A. (2008). CYP725A4 from yew catalyzes complex structural rearrangement of taxa-4(5),11(12)-diene into the cyclic ether 5(12)-oxa-3(11)-cyclotaxane. J. Biol. Chem. 283: 6067-6075.

Routaboul, J.M., Kerhoas, L., Debeaujon, I., Pourcel, L., Caboche M., Einhorn, J., and Lepiniec, L. (2006). Flavonoid diversity and biosynthesis in seed of Arabidopsis thaliana. Planta 224: 96-107.

Ruegger, M., Meyer, K., Cusumano, J. C., and Chapple, C. (1999). Regulation of ferulate-5-hydroxylase expression in Arabidopsis in the context of sinapate ester biosynthesis. Plant Physiol. 119: 101-110.

Ruegger, M. and Chapple, C. (2001) Mutations that reduce sinapoylmalate accumulation in Arabidopsis thaliana define loci with diverse roles in phenylpropanoid metabolism. Genetics. 159: 1741-9.

Rupasinghe, S., Baudry, J., and Schuler, M.A. (2003). Common active site architecture and binding strategy of four phenylpropanoid P450s from Arabidopsis thaliana as revealed by molecular modeling. Protein Eng. 16: 721-731.

Rupasinghe, S.G., Duan, H., and Schuler, M.A. (2007) Molecular Definitions of Fatty Acid Hydroxylases in Arabidopsis thaliana. Proteins 68: 279-293.

Ryan, K.G., Swinny, E.E., Winefield, C., and Markham, K.R. (2001). Flavonoids and UV photoprotection in Arabidopsis mutants. Z. Naturforsch. C. 56: 745-754

Saito, S., Hirai, N., Matsumoto, C., Ohigashi, H., Ohta, D., Sakata, K., 
and Mizutani, M. (2004). Arabidopsis CYP707As encode (+)-abscisic acid 8 '-hydroxylase, a key enzyme in the oxidative catabolism of abscisic acid. Plant Physiol. 134: 1439-1449.

Sakamoto, T., Morinaka, Y., Ohnishi, T., Sunohara, H., Fujioka, S., Ueguchi-Tanaka, M., Mizutani, M., Sakata, K., Takatsuto, S., Yoshida, S., Tanaka, H., Kitano, H., and Matsuoka, M. (2006). Erect leaves caused by brassinosteroid deficiency increase biomass production and grain yield in rice. Nat. Biotechnol. 24:105-109

Salaita, L., Kar, R.K., Majee, M., and Downie, A.B. (2005). Identification and characterization of mutants capable of rapid seed germination at 10 degrees $\mathrm{C}$ from activation-tagged lines of Arabidopsis thaliana. J Exp. Bot. 56: 2059-2069.

Saslowsky, D., and Winkel-Shirley, B. (2001). Localization of flavonoid enzymes in Arabidopsis roots. Plant J. 27: 37-48.

Sarosh, B.R., Wittstock, U., Halkier, B.A., and Ekbom, B. (2010). The influence of metabolically engineered glucosinolates profiles in Arabidopsis thaliana on Plutella xylostella preference and performance. 20:1-9.Chemoecology 20: 1-19.

Sauveplane, V., Kandel, S., Kastner, P.E., Ehlting, J., Compagnon, V., Werck-Reichhart, D., and Pinot, F. (2009). Arabidopsis thaliana CYP77A4 is the first cytochrome P450 able to catalyze the epoxidation of free fatty acids in plants. FEBS J. 276: 719-735.

Schilmiller, A.L., Stout, J., Weng, J.K., Humphreys, J., Ruegger, M.O., and Chapple, C. (2009). Mutations in the cinnamate 4-hydroxylase gene impact metabolism, growth and development in Arabidopsis. Plant J. 60: 771-782.

Schoch, G., Goepfert, S., Morant, M., Hehn, A., Meyer, D., Ullmann, P., and Werck-Reichhart, D. (2001). CYP98A3 from Arabidopsis thaliana is a 3'-hydroxylase of phenolic esters, a missing link in the phenylpropanoid pathway. J. Biol. Chem. 276: 36566-36574

Schoenbohm, C., Martens, S., Eder, C., Forkmann, G., and Weisshaar, B. (2000). Identification of the Arabidopsis thaliana flavonoid 3'-hydroxylase gene and functional expression of the encoded P450 enzyme. Biol. Chem. 381: 749-753.

Schuler, M.A., and Werck-Reichhart, D. (2003). Functional Genomics of P450s. Annu. Rev. Plant Biol. 54: 629-637.

Schuler, M., Duan, H., Bilgin, M., and Ali, S. (2006). Arabidopsis cytochrome P450s through the looking glass: a window on plant biochemistry. Phytochmistry Rev. 5: 205-237.

Seltmann, M.A., Stingl, N.E., Lautenschlaeger, J.K., Krischke, M., Mueller, M.J., and Berger, S. (2010). Differential impact of lipoxygenase 2 and jasmonates on natural and stress-induced senescence in Arabidopsis. Plant Physiol. 152:1940-50.

Serra, O., Soler, M., Hohn, C., Sauveplane, V., Pinot, F., Franke, R., Schreiber, L., Prat, S., Molinas, M., and Figueras, M. (2009) CYP86A33-Targeted Gene Silencing in Potato Tuber Alters Suberin Composition, Distorts Suberin Lamellae, and Impairs the Periderm's Water Barrier Function. Plant Physiol. 149: 1050-1060.

Shibuya, M., Hoshino, M., Katsube, Y., Hayashi, H., Kushiro, T., and Ebizuka, Y. (2006). Identification of beta-amyrin and sophoradiol 24-hydroxylase by expressed sequence tag mining and functional expression assay. FEBS J. 273: 948-959.

Sheahan, J.J. (1993). Differential visualization of transparent testa mutants in Arabidopsis thaliana. Anal. Chem. 65: 961-963.

Shimada, Y., Fujioka, S., Miyauchi, N., Kushiro, M., Takatsuto, S., Nomura, T., Yokota, T., Kamiya, Y., Bishop, G.J., and Yoshida, S. (2001). Brassinosteroid-6-oxidases from Arabidopsis and tomato catalyze multiple C- 6 oxidations in brassinosteroid biosynthesis. Plant Physiol. 126: 770-779.

Shimada, Y., Goda, H., Nakamura, A., Takatsuto, S., Fujioka, S., and Yoshida, S. (2003). Organ-specific expression of brassinosteroid- biosynthetic genes and distribution of endogenous brassinosteroids in Arabidopsis. Plant Physiol. 131: 287-297.

Shimura, K., Okada, A., Okada, K., Jikumaru, Y., Ko, K.W., Toyomasu, T., Sassa, T., Hasegawa, M., Kodama, O., Shibuya, N., Koga, J., Nojiri, H., and Yamane, H. (2007). Identification of a biosynthetic gene cluster in rice for momilactones. J. Biol. Chem. 282: 34013-34018.

Sibbesen, O., Koch, B., Halkier, B.A., and Moller, B.L. (1995). Cytochrome P-450TYR is a multifunctional heme-thiolate enzyme catalyzing the conversion of L-tyrosine to $p$-hydroxyphenylacetaldehyde oxime in the biosynthesis of the cyanogenic glucoside dhurrin in Sorghum bicolor (L.) Moench. J. Biol. Chem. 270: 3506-3511.

Smith, S.A., Beaulieu, J.M., and Donoghue, M.J. (2010). An uncorrelated relaxed-clock analysis suggests an earlier origin for flowering plants. Proc. Natl. Acad. Sci. USA 107: 5897-5902.

Smolen, G., and Bender, J. (2002). Arabidopsis cytochrome P450 cyp83B1 mutations activate the tryptophan biosynthetic pathway. Genetics 160: 323-332

Soliday, C.L., and Kolattukudy P.E. (1978). Midchain hydroxylation of 16-hydroxypalmitic acid by the endoplasmic reticulum fraction from germinating Vicia faba. Arch. Biochem. Biophys. 188: 338-347.

Stewart, J.J., Akiyama, T., Chapple, C., Ralph, J., and Mansfield, S.D. (2009). The effects on lignin structure of overexpression of ferulate 5-hydroxylase in hybrid poplar. Plant Physiol. 50: 621-635.

Stoltzfus, A., Logsdon, J.M.J., Palmer, J. D., and Doolittle, W. F. (1997). Intron "sliding" and the diversity of intron positions. Proc. Natl. Acad. Sci. USA. 94: 10739-10744.

Sun, J.Y., Sønderby, I.E., Halkier, B.A., Jander, G., and de Vos, M. (2010). Non-Volatile Intact Indole Glucosinolates are Host Recognition Cues for Ovipositing Plutella xylostella. J. Chem. Ecol. 35: 1427-1436.

Sugawara, S., Hishiyama, S., Jikumaru, Y., Hanada, A., Nishimura, T., Koshiba, T., Zhao, Y., Kamiya, Y., and Kasahara, H. (2009) Biochemical analyses of indole-3-acetaldoxime-dependent auxin biosynthesis in Arabidopsis. Proc. Natl. Acad. Sci. USA. 31: 5430-5.

Swain, S.M., Singh, D.P., Helliwell, C.A., and Poole, A.T. (2005). Plants with increased expression of ent-kaurene oxidase are resistant to chemical inhibitors of this gibberellin biosynthesis enzyme. Plant Cell Physiol. 46: 284-291.

Swaminathan, S., Morrone, D., Wang, Q., Fulton, D.B., and Peters, R.J. (2009). CYP76M7 is an ent-cassadiene C11alpha-hydroxylase defining a second multifunctional diterpenoid biosynthetic gene cluster in rice. Plant Cell 21: 3315-3325.

Szekeres, M., Nemeth, K., Koncs-Kalman, Z., Mathur, J., Kauschmann, A., Altmann, T., Redei, G. P., Nagy, F., Schell, J., and Koncs, C. (1996). Brassinosteroids rescue the deficiency of CYP90, a cytochrome P450 controlling cell elongation and de-etiolation in Arabidopsis. Cell 85: 171-182.

TAGI (2000). Analysis of the genome sequence of the flowereing plant Arabidopsis thaliana. Nature 408: 796-815.

Takahashi, N., Nakazawa, M., Shibata, K., Yokota, T., Ishikawa, A., Suzuki, K., Kawashima, M., Ichikawa, T., Shimada, H., and Matsui, M. (2005). shk1-D, a dwarf Arabidopsis mutant caused by activation of the CYP72C1 gene, has altered brassinosteroid levels. Plant J. 42: 13-22.

Takei, K., Yamaya, T., and Sakakibara, H. (2004). Arabidopsis CYP735A1 and CYP735A2 encode cytokinin hydroxylases that catalyze the biosynthesis of trans-Zeatin. J. Biol. Chem. 279: 41866-41872.

Tamura, N., Yoshida, T., Tanaka, A., Sasaki, R., Bando, A., Toh, S., Lepiniec, L., and Kawakami N. (2006) Isolation and characterization of high temperature-resistant germination mutants of Arabidopsis thaliana. Plant Cell Physiol. 47: 1081-1094.

Tanaka, K., Asami, T., Yoshida, S., Nakamura, Y., Matsuo, T., and Okamoto, S. (2005). Brassinosteroid homeostasis in Arabidopsis is 
ensured by feedback expressions of multiple genes involved in its metabolism. Plant Physiol. 138: 1117-1125.

Tanabe, S., Ashikari, M., Fujioka, S., Takatsuto, S., Yoshida, S., Yano, M., Yoshimura, A., Kitano, H., Matsuoka, M., Fujisawa, Y., Kato, H., and Iwasaki, Y. (2005). A novel cytochrome P450 is implicated in brassinosteroid biosynthesis via the characterization of a rice dwarf mutant, dwarf11, with reduced seed length. Plant Cell 17: 776-790.

Tanaka, K., Asami, T., Yoshida, S., Nakamura, Y., Matsuo, T., and Okamoto, S. (2005). Brassinosteroid homeostasis in Arabidopsis is ensured by feedback expressions of multiple genes involved in its metabolism. Plant Physiol. 138: 1117-1125.

Tantikanjana, T., Yong, J.W., Letham, D.S., Griffith, M., Hussain, M., Ljung, K., Sandberg, G., and Sundaresan, V. (2001). Control of axillary bud initiation and shoot architecture in Arabidopsis through the SUPERSHOOT gene. Genes Dev. 15: 1577-1588.

Thimmapuram, J., Duan, H., Liu L., and Schuler M.A. (2005). Bicistronic and fused monocistronic transcripts are derived from adjacent loci in the Arabidopsis genome. RNA. 11: 128-38.

Thompson, J.D., Higgins, D.G., and Gibson, T.J. (1994). CLUSTAL W: improving the sensitivity of progressive multiple sequence alignment through sequence weighting, position-specific gap penalties and weight matrix choice. Nucleic Acids Res. 22: 4673-4680.

Thornton, L.E., Rupasinghe, S.G., Peng, H., Schuler, M.A., and Neff, M.M. (2010). Arabidopsis CYP72C1 is an atypical cytochrome P450 that inactivates brassinosteroids. Plant Mol. Biol. 74: 167-181.

Tian, L., Musetti, V., Kim, J., Magallanes-Lundback, M., and DellaPenna, D. (2004). The Arabidopsis LUT1 locus encodes a member of the cytochrome P450 family that is required for carotenoid epsilon-ring hydroxylation activity. Proc. Natl. Acad. Sci. USA 101: 402-407.

Tijet, N., Helvig, C., Pinot, F., Le Bouquin, R., Lesot, A., Durst, F., Salaun, J.P., and Benveniste, I. (1998). Functional expression in yeast and characterization of a clofibrate-inducible plant cytochrome P-450 (CYP94A1) involved in cutin monomers synthesis. Biochem J. 332: 583-589.

Turk, E.M., Fujioka, S., Seto, H., Shimada, Y., Takatsuto, S., Yoshida, S., Wang, H., Torres, Q.I., Ward, J.M., Murthy, G., Zhang, J., Walker, J.C., and Neff, M.M. (2005). BAS1 and SOB7 act redundantly to modulate Arabidopsis photomorphogenesis via unique brassinosteroid inactivation mechanisms. Plant J. 42: 23-34.

Tung, C.W., Dwyer, K.G., Nasrallah, M.E., and Nasrallah, J.P. (2005). Genome-Wide Identification of Genes Expressed in Arabidopsis Pistils Specifically along the Path of Pollen Tube Growth. Plant Physiol. 138: 977-989.

Turk, E.M., Fujioka, S., Seto, H., Shimada, Y., Takatsuto, S., Yoshida, S., Denzel, M.A., Torres, Q.I., and Neff, M.M. (2003). CYP72B1 inactivates brassinosteroid hormones: an intersection between photomorphogenesis and plant steroid signal transduction. Plant Physiol. 133: 1643-1653.

Umezawa, T., Okamoto, M., Kushiro, T., Nambara, E., Oono, Y., Seki, M., Kobayashi, M., Koshiba, T., Kamiya, Y., and Shinozaki, K. (2006). CYP707A3, a major ABA 8 '-hydroxylase involved in dehydration and rehydration response in Arabidopsis thaliana. Plant J. 46: 171-182.

Van de Peer, Y., Fawcett, J.A., Proost, S., Sterck, L., and Vandepoele, K. (2009). The flowering world: a tale of duplications. Trends Plant Sci. 14: $680-688$.

Van Poecke, R.M., Posthumus, M.A., and Dicke, M. (2001) Herbivoreinduced volatile production by Arabidopsis thaliana leads to attraction of the parasitoid Cotesia rubecula: chemical, behavioral, and geneexpression analysis. J. Chem. Ecol. 27: 1911-28.

Vidi, P.A., Kanwischer M., Baginsky S., Austin, J.R, Csucs, G., Dörmann, P., Kessler, F. and Bréhélin, C. (2006). Tocopherol cyclase
(VTE1) localization and vitamin E accumulation in chloroplast plastoglobule lipoprotein particles. J. Biol. Chem. 281: 11225-11234.

von Malek, B., van der Graaff, E., Schneitz, K., and Keller, B. (2002). The Arabidopsis male-sterile mutant dde2-2 is defective in the ALLENE OXIDE SYNTHASE gene encoding one of the key enzymes of the jasmonic acid biosynthesis pathway. Planta 216: 187-192.

Wang, E., Wang, R., DeParasis, J., Loughrin, J.H., Gan, S., and Wagner, G.J. (2001). Suppression of a P450 hydroxylase gene in plant trichome glands enhances natural-product-based aphid resistance. Nat. Biotechnol. 19: 371-374.

Wang, J.W., Schwab, R., Czech, B., Mica, E., and Weigel, D. (2008). Dual effects of miR156-targeted SPL genes and CYP78A5/KLUH on plastochron length and organ size in Arabidopsis thaliana. Plant Cell. 20: 1231-1243.

Watson, C.J.W., Froehlich, J.E., Josefsson, C.A., Chappel, C., Durst, F., Benveniste, I., and Coolbaugh, R.C. (2001) Localization of CYP86B1 in the Outer Envelope of Chloroplasts. Plant Cell Physiol. 42: 873-878.

Wellesen, K., Durst, F., Pinot, F., Benveniste, I., Nettesheim, K., Wisman, E., Steiner-Lange, S., Saedler, H., and Yephremov, A. (2001). Functional analysis of the LACERATA gene of Arabidopsis provides evidence for different roles of fatty acid $\omega$-hydroxylation in development. Proc. Natl. Acad. Sci. USA 98: 9694-9699.

Weng, J.K., Li, X., Stout, J., and Chapple, C. (2008). Independent origins of syringyl lignin in vascular plants. Proc. Natl. Acad. Sci. USA 105: 7887-7892.

Werck-Reichhart, D., and Feyereisen, R. (2000). Cytochromes P450 : a success story. Genome Biol. 1: reviews 3003.

Werck-Reichhart, D., Hehn, A., and Didierjean, L. (2000). Cytochromes P450 for engineering herbicide tolerance. Trends Plant Sci. 5: 116-123.

Williams, P.A., Cosme, J., Sridhar, V., Johnson, E., and Mc Ree, D. E. (2000). Mammalian microsomal cytochrome P450 monooxygenase: structural adaptations for membrane binding and functional diversity. Mol. Cell 5: 121-131.

Winkler, R.G., Frank, M.R., Galbraith, D.W., Feyereisen, R., and Feldmann, K.A. (1998). Systematic reverse genetics of transfer-DNAtagged lines of Arabidopsis. Isolation of mutations in the cytochrome P450 gene superfamily. Plant Physiol. 118: 743-750.

Winkler, R.G., and Helentjaris, T. (1995). The maize Dwarf3 gene encodes a cytochrome P450-mediated early step in Gibberellin biosynthesis. Plant Cell. 7: 1307-1317.

Wittstock, U., and Halkier, B.A. (2000). Cytochrome P450 CYP79A2 from Arabidopsis thaliana L. catalyzes the conversion of L-phenylalanine to phenylacetaldoxime in the biosynthesis of benzylglucosinolate. J. Biol. Chem. 275: 14659-14666.

Xiao, F., Goodwin, S.M., Xiao, Y., Sun, Z., Baker, D., Tang, X., Lenks, M.A., and Zhou, J.M. (2004) Arabidopsis CYP86A2 represses Pseudomonas syringae type III genes and is required for cuticle development. EMBO J. 23: 2903-2913.

Xu, W., Bak, S., Decker, A., Paquette, S.M., Feyereisen, R., and Galbraith, D.W. (2001). Microarray-based analysis of gene expression in very large gene families: the cytochrome P450 gene superfamily of Arabidopsis thaliana. Gene 272: 61-74.

Yamaguchi, S. (2008) A gene encoding steviol synthase and a method for producing steviol. Japanese Patent No. JP2008237110

Zhang, Y., Zhang, B., Yan, D., Dong, W., Yang, W., Li, Q., Zeng, L., Wang, J., Wang, L., Hicks, L.M., He Z. (2011). Two Arabidopsis cytochrome P450 monooxygenases, CYP714A1 and CYP714A2, function redundantly in plant development through gibberellin deactivation. Plant J. 67:342-353.

Zhao, J., Zhang, W., Zhao, Y., Gong, X., Guo, L., Zhu, G., Wang, X., 
Gong, Z., Schumaker, K.S., and Guo, Y. (2007). SAD2, an importin -like protein, is required for UV-B response in Arabidopsis by mediating MYB4 nuclear trafficking. Plant Cell 19: 3805-3818.

Zhou, N., Tootle, T.L., and Glazebrook, J. (1999). Arabidopsis PAD3, a gene required for camalexin biosynthesis, encodes a putative cytochrome P450 monooxygenase. Plant Cell 11: 2419-2428.

Zondlo, S.C., and Irish, V.F. (1999). CYP78A5 encodes a cytochrome P450 that marks the shoot apical meristem boundary in Arabidopsis. Plant J. 19: 259-268.
Zhu, Y., Nomura, T., Xu, Y., Zhang, Y., Peng, Y., Mao, B., Hanada, A., Zhou, H., Wang, R., Li, P., Zhu, X., Mander, L.N., Kamiya, Y., Yamaguchi, S., and He, Z. (2006). ELONGATED UPPERMOST INTERNODE encodes a cytochrome P450 monooxygenase that epoxidizes gibberellins in a novel deactivation reaction in rice. Plant Cell 18: 442-456.

Zybailov, B., Rutschow, H., Friso, G., Rudella, A., Emanuelsson, O., Sun, Q., and van Wijk, K.J. (2008). Sorting signals, N-terminal modifications and abundance of the chloroplast proteome. PLoS One 3: e1994. 